Technion, Israel Institute of Technology

Agricultural Engineering Department

NC State University

Haifa, Israel

Biological and Agricultural Engineering Department

Raleigh, NC, USA

Agricultural Research Organization

Department of ornamental horticulture

Newe Ya'ar, Israel

TRANSPIRATIONAL COOLING OF GREENHOUSE CROPS

Final Report

BARD Research Project No. IS-2538-95R

presented by

Ido Seginer, Technion

Dan H Willits, NCSU

Michael Raviv, ARO

Mary M Peet, NCSU

Haifa, March 2000

BARD Final Scientific Report 


\section{(Cover Page)}

Date of Submission of the report: 31 March 2000

BARD Project Number: IS-2538-95R

Evaluating Panel: Soil and water

Project Title: Transpirational cooling of greenhouse crops

Address \& e-mail of Investigators and Institutions:

\section{$\underline{\text { Investigators }}$}

Principal Investigator:

Ido Seginer

Agricultural Engineering

Haifa, 32000, Israel

segineri\&tx.technion.ac.il

\section{Cooperating Investigators:}

Dan H Willits

Biological \& Agricultural Eng.

Raleigh NC, 27695-7625, USA

willits@eos.ncsu.edu

Michael Raviv

Ornamental Horticulture, ARO

POB 90000, Haifa 31900, Israel

nwyraviv@netvision.net.il

Mary M Peet

Horticultural Science

Raleigh NC, 27695, USA

mary_peet@server.hort.ncsu.edu

\section{$\underline{\text { Institutions }}$}

Technion R\&D Foundation

Technion City

Haifa, 32000, Israel
Vice chancellor for research

NCSU, Box 7003

Raleigh NC, 27695, USA

Scientific director, ARO

Volcani Center

POB 6, Bet Dagan 50250, Israel

Vice chancellor for research

NCSU, Box 7003

Raleigh NC, 27695, USA

Continuation of (Related to) Previous BARD Project:
$\square$ Yes
$x$ No
Number:

Keywords not appearing in the title and in order of importance: Plant spacing, ventilation, ground treatments, evaporative cooling, stress indicators, root-to-shoot ratio.
Budget:
IS: $\$ 187,200$
US: $\$ 150,710$
Total: $\$ 337,910$

Signature

Principal Investigator
Signature

Research Authority, Principal Institution

Publication Summary (numbers) 


\begin{tabular}{|l|c|c|c|c|}
\hline & $\begin{array}{c}\text { Joint } \\
\text { IS/US } \\
\text { authorship }\end{array}$ & $\begin{array}{c}\text { US } \\
\text { Authors } \\
\text { only }\end{array}$ & $\begin{array}{c}\text { Israeli } \\
\text { Authors } \\
\text { only }\end{array}$ & Total \\
\hline Refereed (published, in press, accepted) & & 1 & 1 & 2 \\
\hline Submitted, in review, in preparation & & 1 & & 1 \\
\hline Invited review papers & & & & \\
\hline Book chapters & & & & \\
\hline Books & & & & \\
\hline Master theses & & 2 & 1 & 3 \\
\hline Ph.D. theses & & & & \\
\hline Abstracts & & & & \\
\hline Not refereed (proceedings, reports, etc.) & & & & \\
\hline
\end{tabular}

Patent Summary (numbers)

\begin{tabular}{|l|c|c|c|c|}
\hline & $\begin{array}{c}\text { Israeli } \\
\text { inventor } \\
\text { only }\end{array}$ & $\begin{array}{c}\text { US } \\
\text { inventor } \\
\text { only }\end{array}$ & $\begin{array}{c}\text { Joint } \\
\text { IS/US } \\
\text { inventors }\end{array}$ & Total \\
\hline Submitted & & & & \\
\hline $\begin{array}{l}\text { Issued } \\
\text { (allowed) }\end{array}$ & & & & \\
\hline Licensed & & & & \\
\hline
\end{tabular}

Cooperation Summary (numbers)

\begin{tabular}{|l|c|c|c|c|}
\hline & $\begin{array}{c}\text { From US to } \\
\text { Israel }\end{array}$ & $\begin{array}{c}\text { From } \\
\text { Israel to } \\
\text { US }\end{array}$ & $\begin{array}{c}\text { Together, } \\
\text { elsewhere }\end{array}$ & Total \\
\hline Visits/Meetings & 2 & 2 & 1 & 5 \\
\hline Sabbaticals & & & & \\
\hline Postdoctorates & & & & \\
\hline
\end{tabular}

- Cooperation, briefly explain whether synergistic, complementary or supportive.

Some of each kind: Some transfer of measuring methods, different experimental setups in terms of scale, some support from Newe Yaar to Technion. In terms of exchange of ideas the cooperation was synergistic. 


\section{TRANSPIRATIONAL COOLING OF GREENHOUSE CROPS}

Ido Seginer, Dan H Willits, Michael Raviv, Mary M Peet

\section{Contents}

page

$\begin{array}{ll}\text { Abstract } & 3 \\ \text { Achievements } & 5 \\ \text { Summary report } & 9\end{array}$

\section{Appendices}

\section{A Papers}

A1Constraints and limitations in greenhouse cooling: Challenges for the A1 19

next decade

DH Willits

A2 The effect of canopy density, ventilation rate and evaporative cooling on the transpiration of a greenhouse tomato crop

DH Willits

A3Using chlorophyll fluorescence to model leaf photosynthesis in A3 51

greenhouse pepper and tomato

DH Willits, MM Peet

A4 Measurement of chlorophyll fluorescence as a heat stress indicator in tomato: Laboratory and greenhouse comparisons

DH Willits, MM Peet

A5 Leaf temperature error from heat conduction along thermocouple wires

A5 85

M Tarnopolsky, I Seginer

A6 Ground treatments to relieve heat stress of a sparse greenhouse canopy

A6 95

I Seginer, M Tarnopolsky

C NCSU reports

C1 Setup and general procedures

C1 113

DH Willits, MM Peet

C2 Greenhouse cooling as affected by canopy density, ventilation rate,

evaporative pad cooling, shade and mixing fans

DH Willits

C3 Combining fog and mixing fans with evaporative pad cooling DH Willits

C4 Transpiration as affected by canopy density

DH Willits 


\section{T Technion reports}

$\begin{array}{lll}\text { T1 Setup and general procedure } & \text { T1 } 151\end{array}$

I Seginer, M Tarnopolsky

$\begin{array}{lll}\text { T2 Comparison of plants and evaporimeters } & \text { T2 } 155\end{array}$

I Seginer, M Tarnopolsky

$\begin{array}{lll}\text { T3 Response of plants to ventilation regimes } & \text { T3 } 165\end{array}$

I Seginer, M Tarnopolsky

T4 Cooling by mist

I Seginer, M Tarnopolsky

T5 Water stress experiments

T5 179

I Seginer, M Tarnopolsky

\section{Y Newe Ya'ar reports}

Y1 The effect of root/shoot ratio on specific transpiration of growthchamber grown tomato transplants

M Raviv, S Medina, A Krasnovsky

Y2 The effect of root/shoot ratio on specific transpiration of greenhouse grown tomato transplants

M Raviv, S Medina, A Krasnovsky 


\section{ABSTRACT}

Ido Seginer, Technion, Haifa, Israel

Dan H Willits, Mary M Peet, NC State University, Raleigh, NC, USA

Michael Raviv, ARO, Newe Ya'ar, Israel

\section{Background}

Transplanting vegetable seedlings to final spacing in the greenhouse is common practice. At the time of transplanting, the transpiring leaf area is a small fraction of the ground area and its cooling effect is rather limited. A preliminary modeling study suggested that if water supply from root to canopy is not limiting, a sparse crop could maintain about the same canopy temperature as a mature crop, at the expense of a considerably higher transpiration flux per leaf (and root) area. The objectives of this project were (1) to test the predictions of the model, (2) to select suitable cooling methods, and (3) to compare the drought resistance of differently prepared seedlings.

\section{Procedure}

Plants were grown at several configurations in high heat load environments, which were moderated by various environmental control methods. The difference between the three experimental locations was mainly in terms of scale, age of plants, and environmental control. Young potted plants were tested for a few days in small growth chambers at Technion and Newe Ya'ar. At NCSU, tomato plants of different ages and planting densities were compared over a whole growing season under conditions similar to commercial greenhouses.

\section{Results}

Effect of spacing: Densely spaced plants transpired less per plant and more per unit ground area than sparsely spaced plants. The canopy temperature of the densely spaced plants was lower. Air temperature was lower and humidity higher in the compartments with the densely spaced plants. The difference between species was mainly in the canopy-to-air Bowen ratio, which was positive for pepper and negative for tomato.

Effect of cooling methods: Ventilation and evaporative pad cooling were found to be effective and synergetic. Air mixing turned out to be very ineffective, indicating that the canopy-to-air transfer coefficient is not the limiting factor in the ventilation process. Shading and misting, both affecting the leaf temperature directly, proved to be very effective canopy cooling methods. However, in view of their side effects, they should only be considered as emergency measures.

On-line measures of stress: Chlorophyll fluorescence was shown to accurately predict photosynthesis. This is potentially useful as a rapid, non-contact way of assessing canopy heat stress. Normalized canopy temperature and transpiration rate were shown to correlate well with water stress.

Drought resistance of seedlings: Comparison between normal seedlings and partially defoliated ones, all subjected to prolonged drought, indicated that removing about half of the lowermost leaves prior to transplanting, may facilitate adjustment to the more stressful conditions in the greenhouse.

\section{Implications}

The results of this experimental study may lead to: (1) An improved model for a sparse canopy in a greenhouse. (2) A better ventilation design procedure, utilizing improved estimates of the evaporation coefficient for different species and plant configurations. (3) A 
test for the stress resistance of transplants. 


\title{
ACHIEVEMENTS
}

\author{
Ido Seginer \\ Technion, Haifa, Israel \\ Dan H Willits, Mary M Peet \\ NC State University, Raleigh, NC, USA \\ Michael Raviv \\ ARO, Newe Ya'ar, Israel
}

\section{Accomplishments}

\section{Canopy temperature and transpiration measurements}

Canopy temperature measurement with thermocouples glued to the leaves turned out to be rather successful. Five to ten thermocouples seem to be required for a reliable mean value. A laboratory study quantified the necessary length to be glued, as a function of thermocouple wire diameter and heat conductivity. It has also been established that transpiration rate of container grown plants can be conveniently determined from electronic scales data, even if plants require a support structure.

\section{Effect of canopy density (plant configuration)}

Transpiration was measured over a wide range of canopy density and found to be consistently greater per unit leaf area in sparse canopies. When an essentially complete cover of tomato was compared with essentially isolated plants, the latter transpired over $50 \%$ more than the former on a per plant basis. This is qualitatively in agreement with the prediction of the original model. However, contrary to model prediction that canopy temperature is independent of density, the temperature of the sparse canopy was significantly higher than that of the dense canopy.

The reason for the discrepancy between model and experiment is probably due to the assumption, in the model, that the radiation load per plant in the sparse and dense configurations is the same. In fact the plants in the sparse canopy are not shaded by neighbors, resulting in canopy temperature and transpiration rate that are higher than the current model predicts.

\section{Partitioning to sensible and latent heat}

The ratio between sensible and latent heat in the dissipative fluxes was found to depend considerably on plant species, tomato transpiring rather more than pepper. This also resulted in a stronger cooling effect for tomato. A recurring feature of the data was a canopy-to-air Bowen ratio that is species-dependent but rather insensitive to spacing and to cooling method. The fraction partitioned to latent heat is an important greenhouse ventilation design parameter. Another important modeling element, namely the vapor continuity from plant to greenhouse to outdoors, was also substantiated by the data.

\section{Cooling methods}

Various conventional cooling methods were tested in conjunction with the sparse and dense canopy configurations. Generally speaking, the results agreed with the expectations. Ventilation and evaporative pad cooling were found to be effective and synergetic. Air mixing turned out to be very ineffective, indicating that the canopy-to-air transfer coefficient is not 
the limiting factor in the ventilation process. Cooling by ventilation is more effective in association with more freely transpiring plants (tomato compared to pepper).

Shading and misting, both affecting the leaf temperature directly, proved to be very effective canopy cooling methods. However, in view of their side effects (low light and salt deposition, respectively), they should only be considered as (automatic) emergency measures.

\section{Ground surface treatments}

Several ground surface treatments, intended to reduce the heat load on a sparse canopy, have been compared. Whitewashing reduced air temperature, but had a negligible effect on canopy temperature. Wetting the ground was considerably more effective, but no ground treatment was as good as shading or misting at reducing canopy temperature.

\section{Measurement of Canopy Stress}

Dark adapted $\mathrm{F}_{\mathrm{v}} / \mathrm{F}_{\mathrm{m}}$ chlorophyll fluorescence yield was linked to leaf temperature in tomato, whether measured in a greenhouse or in a laboratory apparatus. Fluorescence was shown to accurately predict photosynthesis in both tomato and pepper. This is potentially useful as a rapid, non-contact way of assessing canopy heat stress. Other, about equally useful water-stress indicators, are normalized canopy temperature and transpiration rate, with an evaporimeter serving as a reference.

\section{Drought resistance of seedlings}

A transient method, where potted seedlings were subjected to a prolonged drought, was tried as a means for assessing the drought resistance of seedlings. Comparison between normal seedlings and partially defoliated ones indicated that removing a considerable fraction (30-50\%) of the lowermost leaves prior to transplanting, may facilitate adjustment to the more stressful conditions in the greenhouse. Specific transpiration rate of the defoliated plants was higher, resulting in a lower leaf temperature and a higher net assimilation rate per sunlit leaf area. These results indicate that the negative long-term effect of defoliation may not be significant.

\section{Deviations from original program}

The original objectives of the project were:

1 Compare canopy temperature and transpiration flux of sparse (young) and dense (mature) canopies, under high heat-load conditions.

2 Find the effects of ventilation rate, artificial evaporative cooling, local canopy velocity and shading, on canopy temperature and transpiration.

3 Develop an environmental control strategy to minimize production losses.

4 Find the relationships between various stress indicators, such as canopy temperature, stomatal resistance, transpiration rate, photosynthesis rate and chlorophyll fluorescence, under high heat load conditions.

$5 \quad$ Evaluate the limiting transpiration rate (a parameter of the model) that different plants (with a range of root/shoot ratios) can sustain. If limit is not distinct, identify limit of well-being of plants with appropriate stress indicator.

6 Devise a standard test for the transpiration capacity of seedlings.

Objectives 1 and 2 were covered rather well, although the large number of cooling methods limited the amount of data for each of them. Objective 3 was only partially 
completed, since a clear quantitative strategy has not been developed. Considerable work has been done on Objective 4, indicating that chlorophyll fluorescence, canopy temperature and transpiration rate can be used as on-line stress indicators. Objective 5 was not achieved, mainly due to technical difficulties. Some progress towards Objective 6 has been made with the transient drought test.

There are several general reasons why we were unable to complete some of the tasks. The project dealt with a second order effect (canopy density), which requires high accuracy measurements. At the same time the environmental conditions were mostly uncontrolled, fluctuating with the weather and introducing considerable data noise. The plant material at Haifa was not sufficiently uniform, and introduced further noise, which could only be removed by multiple replications, more than could be afforded. Finally, Objective 5, as conceived, could not be accomplished due to technical deficiency.

On the other hand, we completed rather comprehensive studies on thermocouple errors and chlorophyll fluorescence, beyond what was originally intended. Given more time for further analysis of the data, additional journal-level reports are likely to follow.

\section{Cooperation}

Cooperation in this study was rather tight. The senior investigators met more than once a year to discuss the work programs, the methodology and the analysis of data. In between meetings e-mail was used extensively for transfer of information. We have not had the time yet to prepare joint papers, but two likely candidates are the drought resistance experiments at Technion and Newe Ya'ar and comparison of the effects of canopy density and of several cooling methods at NCSU and Technion. Qualitatively, the results seem to agree, but more time is required to work out the details. 


\section{SUMMARY REPORT}

Ido Seginer

Technion, Haifa, Israel

Dan H Willits, Mary M Peet

NC State University, Raleigh, NC, USA

Michael Raviv

ARO, Newe Ya'ar, Israel

\section{Background}

Transplanting vegetable seedlings to final spacing in the greenhouse is common practice. At the time of transplanting, the transpiring leaf area is a small fraction of the ground area and its cooling effect is rather limited. Sparsely distributed seedlings may be viewed as a collection of micro-oases, where the dry surroundings of each plant contribute to its energy load via micro-advection. The result is an increased transpirational demand, which may lead to water stress and over-heating of the plants.

A preliminary modeling study (Seginer, 1994) suggested that if water supply from root to canopy is not limiting, a sparse crop could maintain about the same canopy temperature as a mature crop, at the expense of a considerably higher transpiration flux per leaf (and root) area. The objectives of this project were (1) to test the predictions of the model, (2) to select suitable cooling methods, and (3) to compare the drought resistance of differently prepared seedlings.

\section{Methodology}

The study was experimental, utilizing mostly micro-meteorological methods. Plants were grown at several configurations in high heat load environments, which were moderated by various environmental control methods. The difference between the three experimental locations was mainly in terms of scale, age of plants, and environmental control. Young potted pepper and tomato plants were tested for a few days in small growth chambers at Technion and Newe Ya'ar. The Technion chambers were simple, naturally lit units. The Newe Ya'ar chambers were completely controlled units. Similar plants were also tested in a ventilated greenhouse at Newe Ya'ar. At NCSU, tomato plants of different ages and planting densities were compared over a whole growing season under conditions similar to commercial greenhouses. A schematic description of the Technion setup is shown in Figure 1 (=Figure T1.2). 'Sparse configuration' refers to just the core plants (3 pots) and 'dense configuration' refers to core plus perimeter plants (39 pots). 


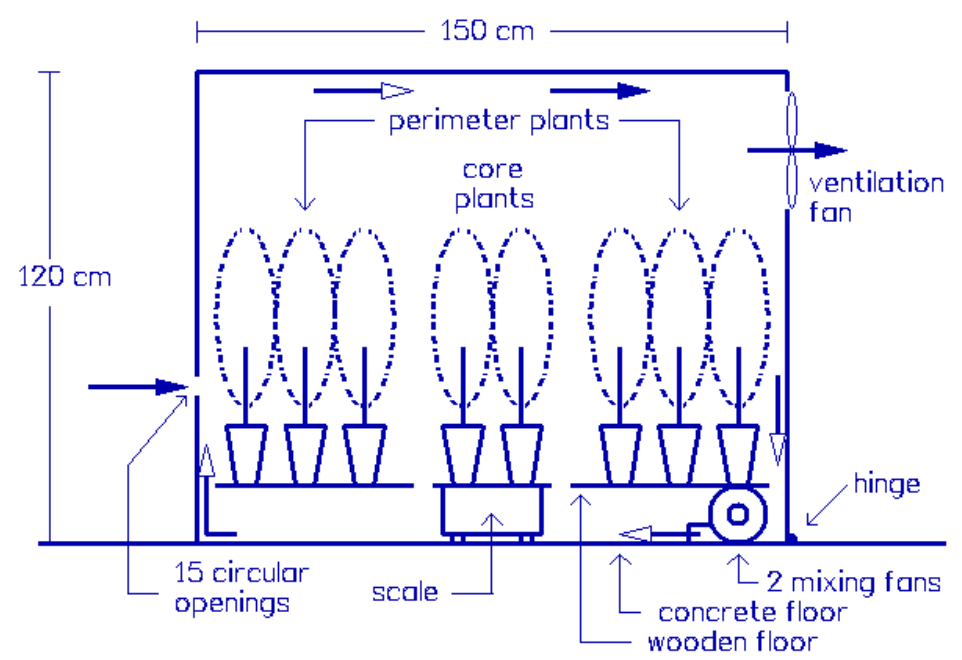

Figure 1: A schematic vertical cross-section of a chamber at Technion, with arrangement of plants and fans. Filled and empty arrows indicate, respectively, ventilation and mixing flows.

At NCSU in 1997, one house ('high-density') was planted at 2.54 plants $\mathrm{m}^{-2}$ on 1 May while the adjacent house ('medium-density') was planted at 2.54 plants $\mathrm{m}^{-2}$ on 1 July. In 1998, one house ('medium-density') was planted at 2.54 plants $\mathrm{m}^{-2}$ and the other ('lowdensity') at 0.44 plants $\mathrm{m}^{-2}$, both on 16 June. In 1999, both houses were planted at 2.54 plants $\mathrm{m}^{-2}$ on 14 June.

Two levels of ventilation were used at NCSU and Technion. Additional cooling methods included evaporative pad, fogging and shading at NCSU and misting, shading and surface treatments at Technion. The transpiration rate, canopy temperature and sometimes other measurements, as well as the aerial environment, were monitored at all locations.

\section{Results}

\section{Effect of spacing}

A large fraction of the total effort was devoted to the effect of spacing on the well being of the plants. A representative result from Technion is shown in Figure 2 (=Figure A6.3). The figure shows the significant canopy cooling effect of the dense configuration (D), compared to the sparse configuration (S) for both pepper and tomato. Additional differences are: lower chamber air temperature and higher humidity when the configuration is dense. The difference between species is mainly in the canopy-to-air Bowen ratio (c-to-i slope), which is positive for pepper and negative for tomato. The transpiration rate (per plant) was significantly higher for the sparse plants: by $30 \%$ and $45 \%$ for pepper at high and low ventilation rates respectively, and by almost $90 \%$ for tomato at the low ventilation rate. 


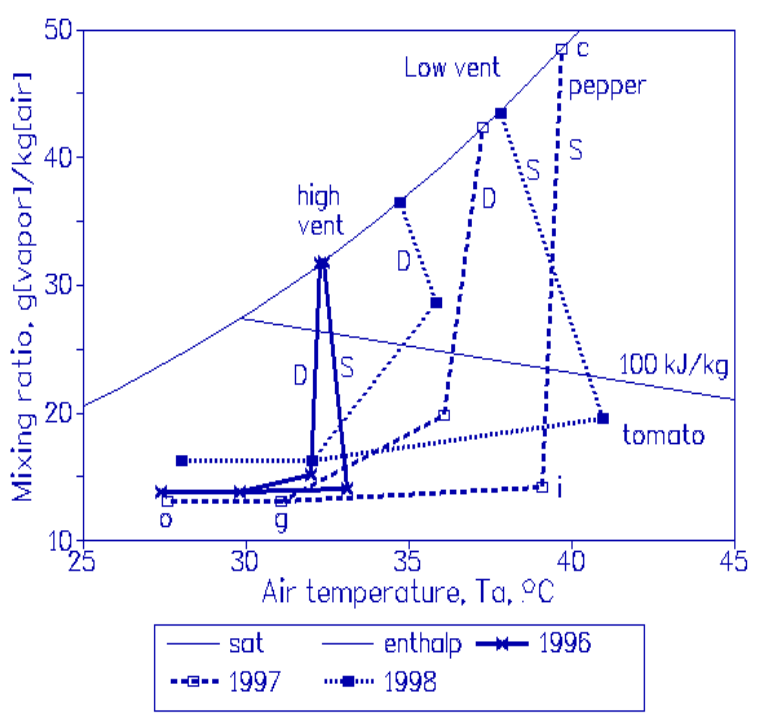

Figure 2: Mean performance of the sparse and dense configurations for two levels of ventilation, two crops (pepper in 1996 and 1997, and tomato in 1998). The results are plotted on a psychrometric chart. D and S denote Dense and Sparse; c, $\mathrm{i}, \mathrm{g}$ and o denote plant canopy, chamber air, greenhouse air and outside air. 'sat' is saturation curve and 'enthalp' is enthalpy line. Ventilation rate was high in 1996 and low in 1997 and 1998.

At Technion, plants of equal size but different spacing were compared, hence leaf area index (LAI, on a ground area basis) was proportional to number of plants per unit area. At NCSU, different LAIs were obtained by contrasting either equally-spaced small (young) and large (old) plants (in 1997) or by spacing differently plants of the same age (in 1998). The resulting transpiration per plant at NCSU is shown in Figure 3, against plant leaf area (a measure of the size or age of the plants). In this figure straight lines through the origin (not shown) represent constant transpiration per leaf area.

The figure shows clearly that both size and spacing have an effect on the transpiration rate (per plant or per leaf area). When the plants are very small, and as long as inter-plant shading is negligible, the results for the $0.44 \mathrm{plant} / \mathrm{m}^{2}$ spacing (diamonds) and the 2.54 plant $/ \mathrm{m}^{2}$ spacing (all other shapes) are similar.

As the plants grow, the canopy of the densely spaced plants closes (when leaf area is about $0.25 \mathrm{~m}^{2} /$ plant) and its transpiration rate per leaf area (slope) decreases due to mutual shading. If weather remained constant over the season (which it did not), then for sufficiently large plants, an asymptotic transpiration per plant is expected to be reached, which is higher for the plants spaced further apart. The Technion results are not directly comparable with Figure 3 (they involved considerably smaller plants and spacing), but they represent a situation qualitatively similar to the data for about $A_{p}=0.6 \mathrm{~m}^{2} /$ plant in Figure 3 .

Leaf temperatures were generally higher (about 1-2 K) in the lower density canopy. One exception was observed early in 1998, when the young plants of both canopies were too small for mutual shading to occur. In that case the leaf temperatures in the low-density canopy were only about $0.2 \mathrm{~K}$ higher than those in the medium-density canopy. 


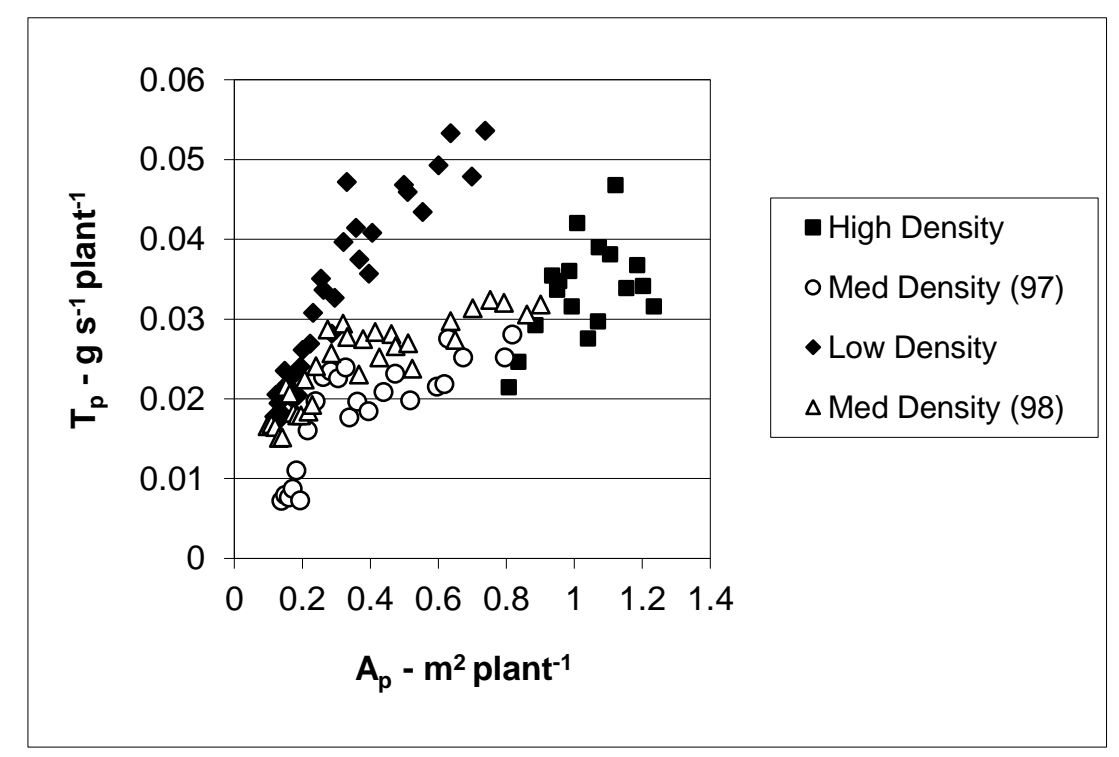

Figure 3: Mean daily transpiration per plant vs. leaf area per plant for 1997 and 1998. Diamonds are for $0.44 \mathrm{plant} / \mathrm{m}^{2}$ and all other symbols are for $2.54 \mathrm{plant} / \mathrm{m}^{2}$.

\section{Effect of cooling methods}

The cooling effect of ventilation is known to be non-linear with rapidly diminishing returns. This was clearly supported by our results at both NCSU and Technion. A Technion example of the effect of ventilation is shown in Figure 2, where pepper was exposed to two levels of ventilation. When ventilation rate is high, increasing it further is not useful, unless it is synergetically combined with another factor, as was observed at NCSU.

The effect of ventilation rate, shade and evaporative pad cooling on transpiration at NCSU was well predicted by a Penman-Monteith relationship. Shade and evaporative pad cooling always reduced transpiration, while ventilation rate generally increased it. Shade was the most effective means of reducing leaf temperature at both NCSU and Technion, but at the presumed expense of reducing photosynthesis. Increasing ventilation rate was very effective when evaporative pad cooling was also used: The higher ventilation rate extended the influence of the evaporative pad further down the length of the NCSU house than was possible at the lower ventilation rate (synergism). Figure 4 (=Figure A1.1) shows the combined effect of evaporative cooling and ventilation at NCSU. The combination of shade, high ventilation rate and evaporative pad cooling produced the lowest leaf temperatures observed at NCSU. The use of mixing fans only marginally reduced leaf temperatures, if at all, at both NCSU and Technion. 


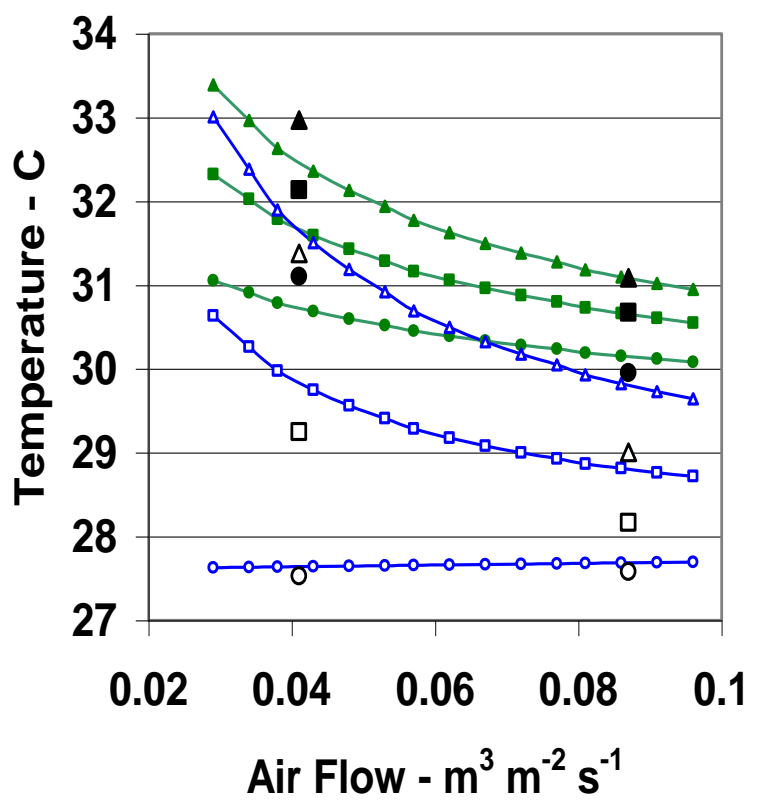

Figure 4: Model predictions and data for the evaporatively cooled case. Filled symbols are canopy temperature, open symbols are air temperature. Circles, squares and triangles are entrance, mid-house and exit locations. The unconnected points were generated from regression equations developed from data collected in the 1997 and 1998 tests.

\section{On-line measures of stress}

The onset of water and/or heat stress may be detected by monitoring one or more of the following variables: canopy temperature, transpiration rate, chlorophyll fluorescence, photosynthesis rate, stomatal resistance, and possibly others. In this study we focused on the first three, fluorescence being measured only at NCSU.

At Technion and Newe Ya'ar the experimental procedure was to stop the irrigation of potted plants and monitor the changes of canopy temperature and transpiration rate of the plants. A representative result is shown in Figure 5 (=Figure T5.4). The results for the waterstressed plants (sub-s) are compared with a reference (sub-r), which in 1998 were irrigated plants and in 1999 - evaporimeters. The initial points of each drought sequence are at the lower-right end of the curves. Generally speaking, the relationships are linear, with some tendency for concavity. These results indicate that there is no clear advantage to either canopy temperature or transpiration rate as indicators of stress.

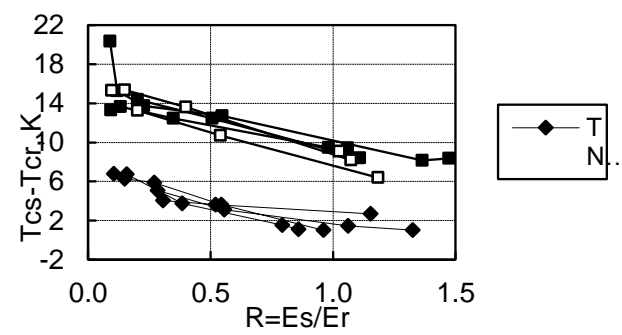


Figure 5: Relationship between the transpiration ratio, $R=E_{s} / E_{r}$ (stressed-toreference), and canopy temperature difference, $\mathrm{T}_{\mathrm{cs}}-\mathrm{T}_{\mathrm{cr}}$ (stressed-to-reference). TND denotes normal $30 \mathrm{~cm}$ tomato plants and THtD denotes similar plants defoliated of the lower half of the leaves.

Measurements of chlorophyll fluorescence and photosynthesis at NCSU were conducted in both laboratory and greenhouse, in an effort to find a rapid, non-contact way of assessing canopy heat stress. Measurements using a LiCor Model 6400 portable photosynthesis meter were conducted in the laboratory using a special cuvette top to hold a fluorescence probe. The method of He and Edwards (1996) was used to calculate photosynthesis from fluorescence yield measurements. The agreement between calculated and measured values of photosynthesis were quite good when stomatal conductance generated from the measured plants was used (Figure $6=$ Figure A3.2a). This suggests that fluorescence yield measurements might be appropriate for real-time determination of photosynthetic activity of the canopy, and therefore serve to aid in the appropriate control of heat stress.

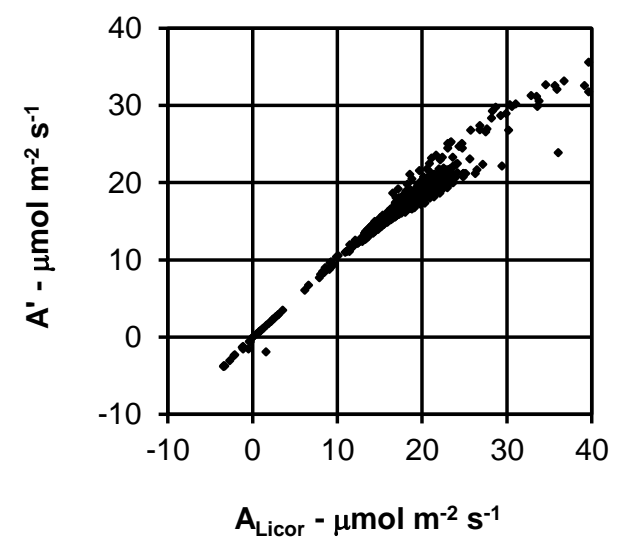

Figure 6: Photosynthesis modeled from chlorophyll fluorescence measurements (A') versus photosynthesis measured using a LiCor Model 6400 photosynthesis

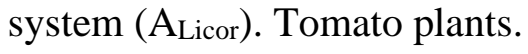

\section{Drought resistance of seedlings}

At Newe Ya'ar and Technion seedlings were subjected to a prolonged drought period (preceding section), so that their drought resistance could be evaluated and compared. In an attempt to evaluate the effect of increased root-to-shoot ratio on the drought resistance of substrate-grown seedlings, some seedlings were partially defoliated and compared to normal seedlings. Figure 5, obtained at Technion, shows no significant difference in terms of temperature and transpiration on a whole plant basis.

Figure 7 (=Figure Y2.2), from the Newe Ya'ar study, shows that there is some delay in water extraction by the stressed defoliated plants (the results for the irrigated plants are not relevant here). Note that transpiration rate is presented in Figure 7 on a leaf area basis. Similar results were also obtained under growth chamber conditions. In Newe Ya'ar, unlike in Technion, canopy temperature of the stressed defoliated plants was 1.5 to $2.0 \mathrm{~K}$ lower compared to the stressed normal plants, resulting in a higher net assimilation rate (Figure 8). This may be an indication that the defoliated plants are more tolerant to drought conditions. 


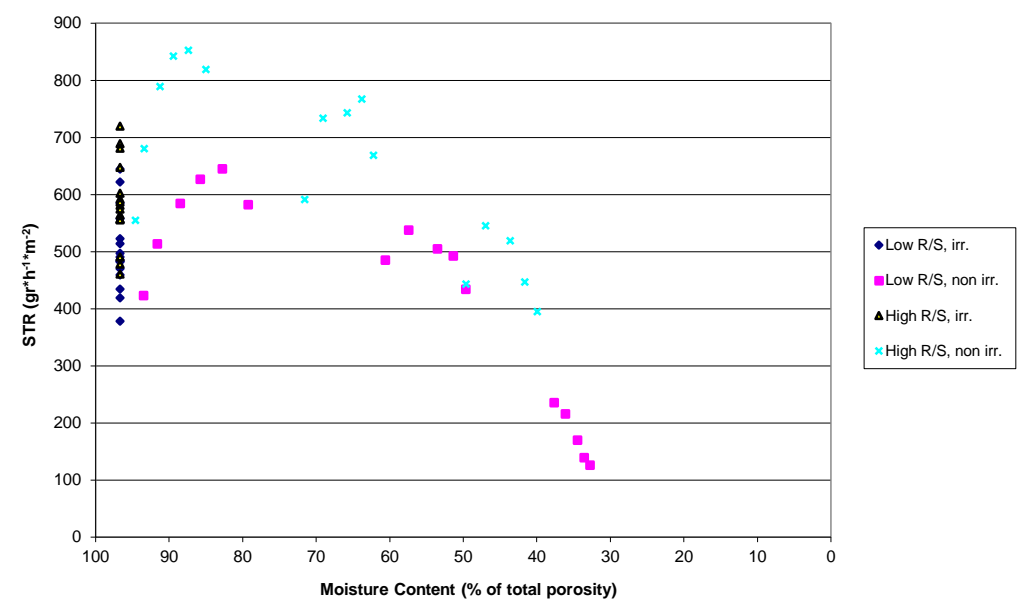

Figure 7: Average specific transpiration rate of irrigated and non-irrigated, normal and partially defoliated plants, as a function of moisture content of the media, over a 3 days period.

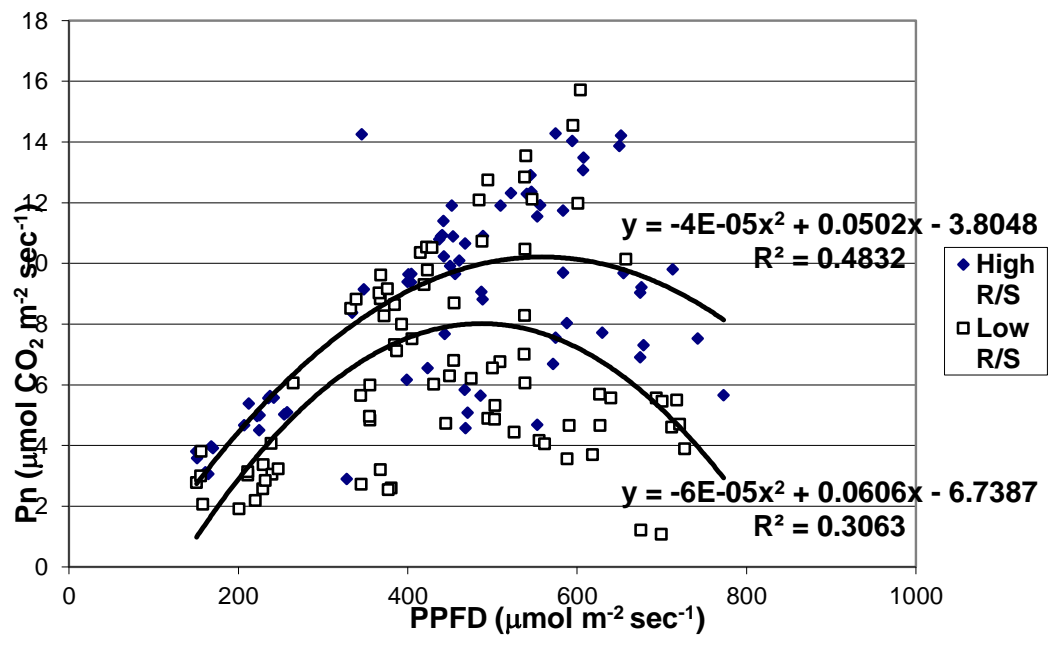

Figure 8: Net photosynthesis of non-irrigated tomato plants as a function of PPFD. Cumulative of 3 experiments. R/S is root-to-shoot ratio. Low R/S are normal plants and high $\mathrm{R} / \mathrm{S}$ are defoliated plants.

\section{Discussion}

The effect of plant spacing, or LAI, is a central element of the project. The experiments substantiated the predictions of the model (Seginer, 1994) that a sparse canopy resulted in a warmer and drier greenhouse air and in a higher transpiration rate. The experiments did not support, however, the prediction that the temperature of the sparse canopy would not be higher than that of the dense canopy. This discrepancy is likely the result of unrealistic geometrical modeling. The model assumes a flat (height-less) canopy, which was obviously not the case in the experiment. The sparse canopy intercepted more solar radiation and longwave per plant than the dense canopy, due to relatively more exposed vertical surfaces. This becomes very clear in view of Figure 1. A two or three dimensional model could presumably reduce the discrepancy.

There is no evidence that the irrigated sparse canopy was stressed to the point that production was harmed. The considerable increase of transpiration rate, and the increase of temperature indicate, however, that under extreme conditions loss of production cannot be 
ruled out.

Several of the cooling devices seem effective in reducing transpiration rate and canopy temperature. In particular, evaporative cooling is effective in reducing the transpiration rate (to prevent water stress) and shading and misting (wetting the surface of the leaves) are effective in reducing canopy temperature. The latter two methods should be applied sparingly, namely automatically, to minimize their negative side effects. Ground surface treatments, such as whitewashing, while effective in reducing air temperature, had no significant effect on canopy temperature. Air mixing, unlike ventilation, was found not to be an effective cooling method.

Several on-line measures of stress were tried. Canopy temperature and transpiration rate respond about equally to low water content in the substrate. Making these measurements on a routine basis in a commercial environment is not easy, but also not out of the question. The chlorophyll fluorescence method has shown some promise for the future.

The transfer of seedlings from the nursery to the greenhouse may require rapid adjustment of the seedlings to the new environment. The transition may be easier if the seedlings are gradually adjusted to the greenhouse environment either before they leave the nursery or when they arrive at the greenhouse. Creating seedlings with a larger root-to-shoot ratio than is required by the nursery environment is a possibility which we investigated by comparing partially defoliated transplants with normal ones. Obviously, if many leaves are removed, the need for water would be greatly reduced and the plant may have more time to adjust to the new surroundings. On the other hand, production may be significantly delayed. The removal of the lower $30-50 \%$ of the leaves, produced a favorable response in Newe Ya'ar and indicates that further improvement can be expected along this line of work.

\section{Potential impact}

This project was mainly intended to be an experimental test of the original model (Seginer, 1994). This was accomplished, resulting in upholding some of the predictions and repudiating others. The results may lead to an improved model, and in any case provide greenhouse designers and researchers with a more accurate view of the greenhouse climate. In particular, the differences we found between species, as well as the effects of canopy density and various cooling methods on the partitioning between sensible and latent heat, may result in better estimates of the 'evaporation coefficient' that is used in ventilation design. The recurrent observation of a canopy-to-air Bowen ratio which is insensitive to spacing and environment, but differs between species, could become a corner stone of a new design method. The transient drought test, which requires further development, may also become a viable test method.

\section{References}

He D, Edwards GE. 1996. Evaluation of the potential to measure photosynthetic rates in $\mathrm{C}_{3}$ plants (Flaveria pringlei and Oryza sativa) by combining chlorophyll fluorescence analysis and a stomatal conductance model. Plant, Cell and Environment 19:1272-1280.

Seginer I. 1994. Transpirational cooling of a greenhouse crop with partial ground cover. Agricultural and Forest Meteorology 71:265-281. 


\section{List of publications}

Seginer I, Tarnopolsky M. 1999. Ground treatments to relieve heat stress of a sparse greenhouse canopy. ASAE Paper 99-4124, 16pp.

Tarnopolsky M, Seginer I. 1999 Leaf temperature error from heat conduction along thermocouple wires. Agricultural and Forest Meteorology 93(3):185-194.

Willits DH, Peet MM. 1999. Using chlorophyll fluorescence to model leaf photosynthesis in greenhouse pepper and tomato. Acta Horticulturae 507:311-317.

Willits DH. 1999. Constraints and limitations in greenhouse cooling: Challenges for the next decade. Proc. International Conference and British-Israeli Workshop on Greenhouse Techniques towards the 3rd Millennium, Haifa, Israel, Sept 5-8, 1999. In print.

Willits DH. 1999. The effect of canopy density, ventilation rate and evaporative cooling on the transpiration of a greenhouse tomato crop. ASAE Paper No. 99-4227. 18pp.

Willits DH, Peet MM. 2000. Measurement of chlorophyll fluorescence as a heat stress indicator in tomato: laboratory and greenhouse comparisons. Submitted to the Journal of Horticultural Science. 


\section{C1}

SETUP AND GENERAL PROCEDURES

Dan H Willits

Biological and Agricultural Engineering, North Carolina State University

Mary M Peet

Horticultural Science, North Carolina State University

\section{Abstract}

Tests conducted in the NCSU greenhouses to examine transpirational cooling in a tomato crop are described below. The facilities were located at the Horticultural Field Laboratory and consisted of two large $(6.7 \times 12.1 \mathrm{~m})$ and two small $(5.2 \times 6.1 \mathrm{~m})$ greenhouses. Most of the testing was performed in the two large houses while the small houses were used primarily for holding replacement plants.

\section{Introduction}

The summer experiments at NCSU were carried out at the Horticultural Field Laboratory while the laboratory experiments were carried out at Weaver Labs. The laboratory tests consisted of fluorescence measurements in tomato (Chapter A4) and photosynthesis and fluorescence measurements in tomato and pepper (Chapter A3). Since materials and methods for these studies have been described in those manuscripts they will not be repeated here. The materials and methods used in summer of 1997 and 1998 have been described in Chapter A2, but will be repeated here as a basis for comparing them to the procedures used in the summer of 1999.

\section{Facilities Description}

The greenhouses were part of a range of different size houses (Figure C1.1). The large houses (T1 and T2) were free standing quonsets, separated from neighboring houses by approximately $3 \mathrm{~m}$. The small houses (T3 and T4) were connected at the gutter to identical houses, environmentally separated from each other by single sheets of polyethylene. The large houses were separated from the small houses by a distance of approximately $20 \mathrm{~m}$ and from each other by a distance of approximately $6 \mathrm{~m}$.

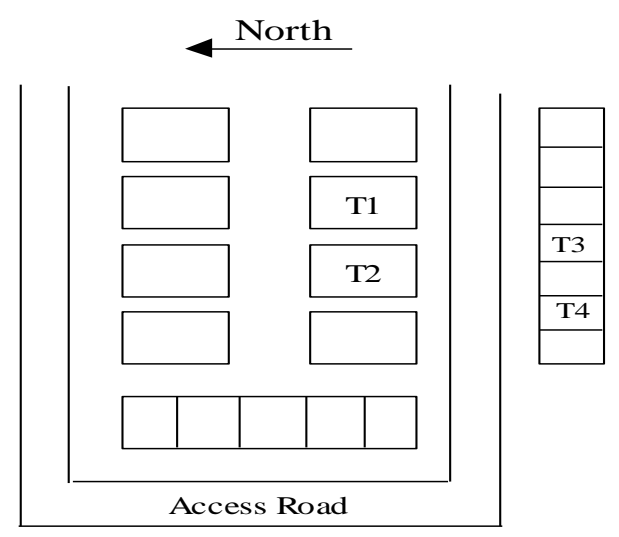

Figure C1.1: Overall arrangement of greenhouse range. 
The large houses were $6.7 \times 12.1 \mathrm{~m}$, double-polyethylene covered quonset-style and the small houses were $5.2 \times 6.1 \mathrm{~m}$, double-polyethylene covered gutter-connected style. The small houses were used for summer environmental data collection only during 1997. No treatments were imposed in the houses at that time, and the data were used only as a check to insure that observations in the two larger houses were reasonable. During the summer of 1998, a mishap in the large houses during startup compromised a large number of plants, such that replacements from the two small houses were necessary in order for testing to continue. During that summer, and again in 1999, the small houses served only as holding areas for plants to be used as replacement for dead and dying plants in the large houses. No data were collected or analyzed from these houses.

The small houses did serve as test houses during the fluorescence testing conducted during the fall of 1997 and the spring of 1998 (Chapter A4) but the remainder of the discussion will focus on the large houses and the treatments imposed in them during the 3 summers.

The large houses were modified to eliminate moisture storage in the soil by placing a $1.1 \mathrm{~mm}$ thick EPDM (ethylene, propylene, diene monomer) waterproof barrier over the top of finely crushed gravel and a black woven landscape cloth placed over the top. Drainage ditches were installed along the sides and across each end of the outside of each house to prevent the entry of water into the houses. Ditches were also dug along the inside of each longitudinal wall. The floors were sloped on a 3\% grade from the center of each house to the ditches on either side to accommodate bag runoff. The egress of each of the inner ditches was blocked to within 1.27 $\mathrm{cm}$ of the bottom of the ditch to allow water to flow out while minimizing the flow of air into the house during venting.

The cooling equipment consisted of two exhaust fans (one two-speed and one single-speed) capable of producing several levels of ventilation rates up to $0.087 \mathrm{~m}^{3} \mathrm{~m}^{-2} \mathrm{~s}^{-1}$, plus an evaporative pad system sized for a pad face velocity of $1.27 \mathrm{~m} \mathrm{~s}^{-1}$ at the highest ventilation rate. During the winter of 1997-98, the fans were tested in a test chamber designed in accordance with ASHRAE Standard 51-75. Flow vs. pressure drop curves were developed from the data and used with Setra model 264 differential pressure transducers mounted in the greenhouses to estimate air flow.

\section{Data Collection}

Environmental control and data collection were handled separately. Environmental control was via personal computer connected to a Fluke Helios I front-end for data input and four Opto 22 relay racks for output. Most of the data collection was via a personal computer connected to a separate Fluke Helios I (expanded to handle 140 channels); however, the control computer also recorded run times of the equipment, control temperatures and solar radiation (every min), and actual irrigation times once per day. The remaining data were recorded as 30-min averages of 1-min readings in 1997 and 10-min averages of 1-min readings in 1998 and 1999.

Figure C1.2 shows the layout of the various sensors for the three years. Solar radiation on a horizontal surface at the top of the canopy was measured using an Epply model 8-48 black and white pyranometer mounted at the top of the evaporative pad on the north wall of each house. Outside solar radiation was measured using an identical pyranometer mounted at the top of the north end of one of the houses 


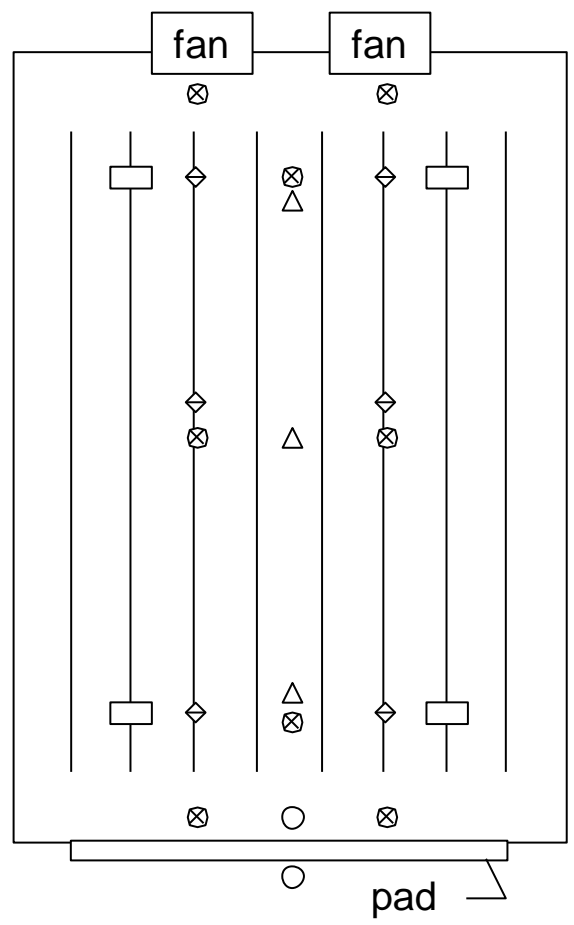

(a) 1997 Configuration

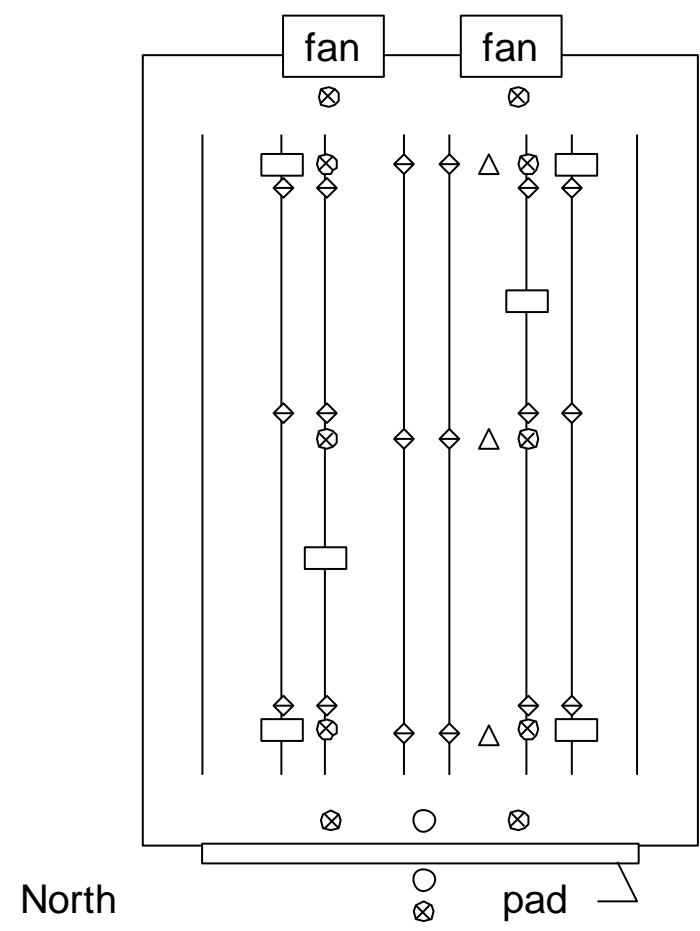

(b) 1998-99 Configuration

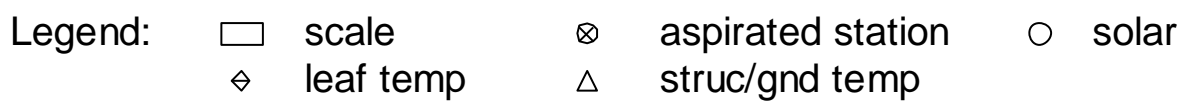

Figure C1.2: Greenhouse and instrumentation layout for the three years. The vertical lines are plant rows and the symbols show the approximate location of various sensors.

\section{Air Temperature and Humidity}

In 1997, aspirated dry-bulb/wet-bulb stations were constructed of $1.25 \mathrm{~cm}$ plywood with 10 $\mathrm{cm} \times 10 \mathrm{~cm}$ aspirator fans mounted at one end and two $4 \mathrm{~cm}$ PVC elbows in the other. The temperatures were measured by placing a 24 ga., type-T thermocouple in the center of the exit of each elbow. One junction was bare and other was wrapped in a cotton sock wet by a drip irrigator fed by a constant-head reservoir. To minimize axial conduction error, each junction was attached to approximately $100 \mathrm{~cm}$ of $24 \mathrm{ga}$. lead wire coiled inside the aspirated box before being connected to the 20 ga. extension wire leading outside. Locations of the stations in the large houses are shown in Figure C1.2, along with the approximate locations of some of the other instrumentation.

In 1997, the two aspirated stations at the air entrance end of each house were located at the top of the pads, with $4 \mathrm{~cm}$ PVC pipes extending down from the elbows to the middle of the opening. Four stations were evenly distributed throughout each canopy. An additional station was located at the entrance to each exhaust fan mounted at the top of the fan with PVC 
pipes extending down to the middle of the fans. One station was located outside approximately $5 \mathrm{~m}$ from the air entrance and about $3 \mathrm{~m}$ off the ground.

In 1998, two additional stations were added in each canopy area and one immediately outside the air entrance. Also in 1998, the PVC uptake pipes of the fan stations in the large houses were extended to the bottom of the fans, the ends capped and holes drilled along the length of the pipes on the sides facing the air flow. The holes were uniformly spaced from top to bottom of the fan entrance. This was done in response to an observation in 1997 that the air entering the fans could become stratified when the plant canopy height was in the vicinity of the fan entrance. In both years, the heights of the canopy stations in all houses were adjusted to keep the air intakes approximately mid-canopy. The air entrance and air exit stations were left in the same position throughout testing. A final change in 1998 was that the wet bulb thermocouples in the large houses were replaced with Vaisala model HM50-U capacitance type relative humidity sensors.

In 1999, location and numbers of the aspirated stations were the same, except that wet bulb thermocouples were added back to those stations located at the air entrance and air exit ends of the houses.

\section{Structure and Floor Temperature}

In all three years, structure temperatures were measured by placing $2 \mathrm{~cm} \times 15 \mathrm{~cm}$ strips of 2 mil, clear polyethylene tape on the underside of 3 of the main structural bows at the apex of the house, one at the air-entrance end, one in the middle and one at the exhaust end of each house. The locations were nearly on the longitudinal centerline of the house. Lengths of approximately $15 \mathrm{~cm}$ of Type-T, 36 ga. thermocouple wire were twisted together at one end to produce junctions $25 \mathrm{~mm}$ in length and soldered. The resulting thermocouples were glued to the plastic strips with contact cement and covered with a second layer of tape for protection. Extension wires ( $20 \mathrm{ga}$.) were attached to each thermocouple and taped to the underside of the member to minimize thermal conduction error.

A similar procedure was used for the floor temperatures. Three thermocouples with dimensions equal to those above were glued to the woven landscape cloth with contact cement in 3 locations, one at the air-entrance end, one in the middle of the house and one at the exhaust end. A $25 \mathrm{~cm}$ x $25 \mathrm{~cm}$ layer of landscape cloth was glued over each thermocouple, using contact cement, to minimize radiation error and to make the surface similar to the rest of the floor.

\section{Leaf Temperature}

In all years, leaf temperatures were measured by gluing $0.077 \mathrm{~mm}$ diameter, type $\mathrm{K}$ thermocouples to the underside of leaves in the upper canopy on selected plants. The thermocouples were made by stripping the insulation approximately $15 \mathrm{~mm}$ back from the ends, twisting the pair together for a distance of approximately $3 \mathrm{~mm}$ and soldering. The resulting thermocouple consisted of about $10 \mathrm{~mm}$ of bare leads with about $3 \mathrm{~mm}$ of twisted wire. The entire $13 \mathrm{~mm}$ was coated on one side with a thin layer of contact cement and the solvent allowed to evaporate for a period of about $4 \mathrm{~min}$. The thermocouple was then gently pressed onto the bottom surface of the leaf.

In 1997, temperatures were measured on each of 6 sample plants spaced uniformly throughout each house. Initially, only two leaf thermocouples per plant could be applied to the small 
plants because the number of suitably sized leaves was insufficient to support a third. On 5 August, the third thermocouple was attached and from then on, one thermocouple was located on the east side of each plant, one on the south side and one on the west, all three in the upper canopy. The thermocouples were moved up at approximately 2-week intervals, or earlier if they became noticeably shaded. In 1998, 18 plants were used in the dense canopy house, one leaf on each plant. The plants were located on alternate sides of the double row such that half the leaves had eastern exposure and half western exposure. In the sparse canopy house there were not enough plants to form double rows (or even single rows), but the layout was such that 9 plants were located in the same locations relative to air flow as in the dense canopy house, with approximately the same relative row locations. Two leaves were used on each of these plants, one on the eastern side and one on the western. In 1999, leaf temperatures were measured as in the dense canopy house in 1998.

The thermocouples were inspected each day between 700 and 800 h (EST). Repairs were made before $900 \mathrm{~h}$ to minimize loss of data for that day. Notes from the log were used to compile a thermocouple status database. Thermocouples which were partially or completely detached or located near necrotic tissue (from occasional solvent damage) were excluded from the dataset for the entire previous day.

\section{Transpiration}

Scales were used to measure the moisture loss of selected plants. The scales chosen were model S-AL25 (Digi Matex, Inc. Norwood, NJ) OEM platform scales with an output of about $0.00090 \mathrm{~V} \mathrm{~g}^{-1}$ using 12 volt excitation. The scales were temperature-compensated between $10 \mathrm{C}$ and $40 \mathrm{C}$. Testing of three units revealed repeatable temperature drifts of $-0.021 \mathrm{~g} / \mathrm{C}$, $0.077 \mathrm{~g} /{ }^{\circ} \mathrm{C}$ and $-0.174 \mathrm{~g} /{ }^{\circ} \mathrm{C}$. Since the largest expected error over a $20{ }^{\circ} \mathrm{C}$ swing was only 3.5 $\mathrm{g}$ the compensation was considered to be acceptable. The scales were found to be linear well beyond the specified capacity of $11 \mathrm{~kg}$. Calibration was performed with excitation voltages ranging between 10 and $14 \mathrm{~V}$, with individual calibration equations being developed for each scale as a function of weight and excitation voltage measured at the load cell.

In 1997 and 1998, each plant placed on a scale was left unsupported until the height of the plant reached about $1 \mathrm{~m}$ above the ground. After this time, it was suspended from an overhead support wire in a manner similar to the other plants, but in such a way as to allow the bag (filled with media and roots) to rest unrestricted on the scale. In this way, the scales measured the change in weight of the bag, which was assumed to represent the moisture transpired by the plant. Periodic calibrations were performed by placing 100, 200 and $300 \mathrm{~g}$ weights directly on the bags and recording the output of the scale. The results showed that the height of the plant had no effect on the ability of the scales to measure changes in the weight of the bag.

It was assumed that changes in weight of the stems and leaves would be small and that the change due to decreasing turgor in the morning would be compensated for by the change due to increasing turgor in the afternoon. A test of this hypothesis was conducted at the end of the 1998 season, where the fresh and dry weights of $3 \mathrm{~m}$ tall plants (excluding roots) were taken at $700 \mathrm{~h}, 1200 \mathrm{~h}$ and $1500 \mathrm{~h}$ (EST), 3 plants at each harvest. On average, the plants lost about $2.5 \%$ of their weight $(35 \mathrm{~g})$ from $700 \mathrm{~h}$ to $1200 \mathrm{~h}$, regaining approximately $1.5 \%(21 \mathrm{~g})$ by $1500 \mathrm{~h}$. This compares to total weight changes of about $600 \mathrm{~g}$ from 700 to $1200 \mathrm{~h}$ and about $400 \mathrm{~g}$ from 1200 to $1500 \mathrm{~h}$. This suggests that the scales might have over-estimated transpiration rates by as much as $6 \%$ in the morning and under-estimated by as much as $5 \%$ in the afternoon. The net effect over the day is sufficiently small as to be considered negligible. The data required smoothing to remove the noise from wind-induced vibration and incidental contact of the plants by personnel. Smoothing was accomplished in several steps: 1) 
eliminating the irrigation periods from the data using the irrigation times recorded by the control computer; 2) replacing those weights with a positive or negative change in voltage in excess of $50 \mathrm{mv}$ from the previous weight with averages of neighboring readings; 3) calculation of simple two point differences from adjacent weights; 4) replacing each difference by a 5-point moving average of the neighboring differences; and 5) averaging the 5 -point averages over 30 minutes periods (10 $\mathrm{min}$ in 1998) matching the environmental datalogging intervals.

Most of the filtering steps were required for the data from the young plants, which was contrary to expectation. Not only was the absolute value of the noise for the young plants about twice as much as in the mature plants (before filtering), the lower total transpiration rates of the young plants exacerbated the problem by making the relative noise 4 times as much (before filtering) as in the mature plants. Filtering reduced the relative noise in the young plants to about 1.3 times as much as in the mature plants.

Transpiration per unit leaf area was determined for each scale plant by dividing the $30 \mathrm{~min}$ averages (10 min in 1998) of the weight differences by leaf area estimated for that plant on that day. Specific transpiration for each house was estimated by averaging the values from the scale plants. In 1997, 4 scales were used in each house. In 1998, two scales were added to each house, one placed third of the way down the house and one placed two thirds of the way down the house.

In 1999, the suspension system for the scale plants was redesigned. Instead of supporting the plants from the overhead wire, a support column was constructed from sections of $5 \mathrm{~cm}$ dia PVC pipe fastened at the bottom to a $1 \mathrm{~cm}$ thick PVC plate. The plate was placed on the scale and the plant bag placed on top of that. As in previous years, no support was needed until the plants reached about $1 \mathrm{~m}$ off the ground. At that time the plants were secured to the PVC support column, and as the plants grew the columns were lengthened by adding sections. In this way, the scale measured the entire weight change of the plant.

\section{Leaf Area}

In 1997, leaf areas were estimated by measuring overall lengths and widths of each compound leaf on 10 sample plants in each house (four of which were on the scales) at 3 times during the study: 1) shortly after the start of testing (7 - 9 July), 2) when the mature plants reached the support wires (24 - 28 July), and 3) at the end of testing (10 - 12 September). During the 24 - 28 July period, the leaves up to the first viable cluster on the mature plants were removed and run through the LiCor leaf area meter (Model 3100) for the purposes of adjusting the totals. At the end of testing, all leaves were run through the leaf area meter in addition to measuring their lengths and widths. The data from the leaf area meter was then regressed against the length and width data to produce leaf area estimates for each of the 10 plants. These were combined with the data from the initial and mid-season measurements to estimate the leaf area of each of the sample plants at three points during the study. These estimates were then regressed against Julian date to produce equations of leaf area as a function of time for each of the sample plants. Averages of those estimates were used to calculate LAI, based on total greenhouse floor area, as a function of Julian date.

In 1998, leaf areas were again estimated 3 times, 1 July, 28 July and 25 August. Seven plants were used to represent the sparse canopy house (6 located on scales and the $7^{\text {th }}$ located at random). Eleven plants were used to represent the dense canopy house (6 located on scales and the remaining 5 uniformly spread across the house). On 1 July, lengths and widths of both individual and compound leaves were recorded on all the sample plants. On 28 July and 25 August, individual and compound dimensions were recorded on only $30 \%$ of the sample 
plants and compound dimensions recorded on the remainder. At the end of testing, leaves from all of the sample plants were removed, the compound and single or single-only leaf dimensions measured, depending upon how the plant had been measured previously, and then the individual leaves were run through the leaf area meter. Regressions were again used to produce the appropriate $L A I$ estimates for the canopy.

In 1999, the procedure was the same as in 1998, but the frequency was increased to every two weeks using 10 plants per house.

\section{Air Velocity}

Air velocities in and above the canopy were measured in two years, 1997 and 1998. Velocities were measured using a hot-wire anemometer (model 441S, Kurtz Instruments, Inc., Monterey, CA) at 8 heights starting at $0.3 \mathrm{~m}$ and ending at $2.4 \mathrm{~m}$ up from the floor. The velocities were measured in each house and in each aisle at three locations, at the air inlet, the middle and at the air exit positions. In 1997, velocity measurements were conducted every two weeks throughout the summer, each time both with and without the evaporative pad running. Eight measurements were made at the lower ventilation rate and 4 at the higher ventilation rate. In 1998, only two measurements were made in each house, both near the start of testing, one with the mixing fans on and one with them off.

\section{Plant Culture}

In 1997, 208 tomato plants (cv. Trust) were transplanted into one of the houses (mature plant house) on 1 May. On 1 July, an additional 208 plants were transplanted into the second (young plant house). The plants were distributed in 8 rows of 28 plants each, $0.8 \mathrm{~m}$ between row centers and $0.4 \mathrm{~m}$ between plants in the same row. Plants in the mature plant house reached the support wires (approx. $2.1 \mathrm{~m}$ high) at the end of July and were kept at that height for the remainder of the study by gradually lowering the plants. At the time of the first lowering, all leaves up to the first viable cluster were removed to prevent them from becoming entangled in the neighboring plants and irrigation lines as they were lowered.

In 1998, 208 plants were transplanted into one of the houses (dense canopy house) on 16 June and 36 into the other (sparse canopy house). The plants did not reach the support wire until nearly the end of testing. Row configurations were slightly different than in 1997. The two outer rows were left in the same location, but the center 6 rows were repositioned to reduce the 3 center-to-center spacings to $0.5 \mathrm{~m}$ while widening the remaining 4 to approximately 1 $\mathrm{m}$. The narrow aisles were used to route drip irrigation headers and instrumentation cables while the wider aisles were used as walkways. In the dense canopy house, the within row plant spacings remained the same as in 1997. In the sparse canopy house, the plants were evenly distributed throughout the house, using the row locations as guides. Each double row contained 9 plants evenly spaced. One of the outside rows contained 4 plants, the other 5 . Only senescent leaves at the bottom of the plants were removed during testing.

In 1999, 208 plants were transplanted into each house on 14 June. Spacing was the same as in the dense canopy house during 1998.

During all three years, the plants were grown in 18.9 liter black polyethylene bags (HydroGardens, Colorado Springs, Colo.) filled with 50\%, by volume, ProMix BX (Premier Brands, New Rochelle, N.Y.) and 50\% aged, $95 \mathrm{~mm}$ mesh screened pine bark. Fertilization was automatic with each watering through drip emitters. Concentrations were (in ppm) 125 N, 45 P, 195 K, $185 \mathrm{Ca}$ and $44 \mathrm{Mg}$. During 1997, waterings were 4 times per day but during 
1998 only a single watering was performed each day. In 1997, irrigation quantities were managed by manually adjusting parameters in the computer program to increase or decrease the volume delivered. During 1998 and 1999, the transpiration data from 1997 was used to develop an algorithm for estimating the volume needed per plant based on solar radiation, air temperature and plant size.

\section{Special Equipment}

Mixing Fans. In 1998, six three-speed, $30 \mathrm{~cm}$ diameter fans (Air King model 9212) were mounted in each house to increase local air velocities in the canopies, 3 at the air-entrance end of the house and 3 midway down the length of the house. Preliminary testing showed that air velocities of approximately $0.1 \mathrm{~m} / \mathrm{s}$ could be maintained within about $40 \mathrm{~cm}$ of the axes of the fans for about $6 \mathrm{~m}$. Based on that information, fan heights (and leaf thermocouple locations) were adjusted on a weekly basis so that the axes of the fans were within $30 \mathrm{~cm}$ of the lowest thermocouple and that both were kept within $40 \mathrm{~cm}$ of the average top of the canopy. Canopy air velocities were measured weekly to insure that conditions were maintained as expected.

Fogging. In 1999, 12 low-pressure fogging nozzles (model 93512, Arizona Mist, Tempe, AZ), each delivering $0.042 \mathrm{lpm}$, were mounted in each house. The nozzles were installed in manifolds made of $2 \mathrm{~cm}$ PVC pipe, three nozzles per manifold, four manifolds per house. The longitudinal axes of the manifolds were $90^{\circ}$ to the longitudinal axis of each house, supported at about $2.1 \mathrm{~m}$ from the floor. The manifolds were space approximately $3 \mathrm{~m}$ apart down the length of each house starting at the air entrance end. A three-speed, $30 \mathrm{~cm}$ dia fan (Air King model 9212) was mounted directly behind each nozzle of the $1^{\text {st }}$ and $3^{\text {rd }}$ manifolds. The fans were used to keep the fog droplets suspended in the air as long as possible so as to minimize wetting of the floor and canopy.

Shade Cloth Misting. Also in 1999, provisions were made to apply mist the outer surface of the black shade cloth used in the final experiment (described below). Micro-irrigation nozzles (model AJS-40 and AJH-40, Wade Mfg. Co., Fresno, CA) were used, each nozzle supplying $9.5 \mathrm{ml} \mathrm{s}^{-1}$ at $68.9 \mathrm{kPa}$. Fourteen nozzles were mounted into two PVC lines, 7 nozzles each, running the length of the house. They were placed on the house spaced approximately 1.5 meters apart on either side of the ridge. The PVC lines were fed from a pressure regulated line and controlled to activate $30 \mathrm{~s}$ out of every $3 \mathrm{~min}$ whenever the outside solar radiation level was above $400 \mathrm{~W} \mathrm{~m}^{-2}$.

\section{Experimental Design}

In 1997, combinations of two levels of ventilation rate $\left(0.041 \mathrm{~m}^{3} \mathrm{~m}^{-2} \mathrm{~s}^{-1} \mathrm{vs} .0 .087 \mathrm{~m}^{3} \mathrm{~m}^{-2} \mathrm{~s}^{-1}\right)$ and two levels of evaporative pad cooling (full and none) were imposed in the two large houses (no treatments were imposed in the small houses), each with a different canopy density (see Plant Culture above). From 1 - 7 July, identical conditions were imposed in both houses (evaporative cooling on, ventilation rate $=0.041 \mathrm{~m}^{3} \mathrm{~m}^{-2} \mathrm{~s}^{-1}$ ). From $8-15 \mathrm{July}$, either ventilation or evaporative cooling levels were different between the houses, with the alternate condition being imposed on the adjacent day.

From 16 July through 22 August, the conditions were again identical in each house on a given day, but the conditions were changed on a 7-day cycle. On four days of the cycle $(2,4,6$ and 7) the ventilation rate was $0.041 \mathrm{~m}^{3} \mathrm{~m}^{-2} \mathrm{~s}^{-1}$ and evaporative pad cooling was on. On the first day evaporative pad cooling was off, on the $3^{\text {rd }}$ day, the ventilation rate was increased to 
$0.087 \mathrm{~m}^{3} \mathrm{~m}^{-2} \mathrm{~s}^{-1}$, and on the $5^{\text {th }}$ day the evaporative cooling was turned off and the ventilation rate was increased to $0.087 \mathrm{~m}^{3} \mathrm{~m}^{-2} \mathrm{~s}^{-1}$.

Starting on 23 August, the above schedule was modified to conform to the needs of some preliminary fluorescence testing. During two days, both houses were run with the low ventilation rate and no evaporative pad cooling, followed by 1 day each at low and high ventilation rates, both with evaporative pad cooling. During eight of the next 10 days, one house was run at the low ventilation rate and the other at the high ventilation rate, both with evaporative pad cooling, with the ventilation rates alternating between houses on a daily basis. During the final two days, ventilation rates were again different in the houses, switching on alternate days, but no evaporative pad cooling was used. Table C1.1 summarizes the number of days the houses spent in each treatment over the whole summer.

Table C1.1: Number of days each cooling treatment was imposed in the two houses during the summer of 1998.

\begin{tabular}{lll}
\hline Vent Rate & $\begin{array}{l}\text { Evaporative } \\
\text { Pad }\end{array}$ & Days \\
\hline 1 & 1 & 32 \\
1 & 0 & 12 \\
2 & 1 & 16 \\
2 & 0 & 7 \\
\hline
\end{tabular}

In 1998, testing began 1 July with the mixing fans turned on in both houses every other day through 14 July. Shade treatments were added starting 15 July and continued through 11 August. The shade cloths were identical black polypropylene knitted shade cloths, rated at $50 \%$ shade, alternately applied and removed to both houses simultaneously in 4-day cycles ( 2 days on and 2 days off), while mixing fan operation continued to be alternated on a daily basis. Care was taken to stagger the application of the fan treatments so that the fans were not always on (or off) following a period of shade or non-shade.

The mixing fans were taken down on 12 August, that treatment being replaced with two levels of ventilation rate starting on 13 August. Again, the shade cloths were applied to both houses on a 4-day cycle ( 2 days on and 2 days off) while ventilation rate was alternated on a daily basis between $0.041 \mathrm{~m}^{3} \mathrm{~m}^{-2} \mathrm{~s}^{-1}$ and $0.087 \mathrm{~m}^{3} \mathrm{~m}^{-2} \mathrm{~s}^{-1}$. Once again, care was taken to insure that each ventilation rate followed a shade treatment or non-shade treatment an equal number of times. The number of days both houses spent in each treatment is summarized in Table C1.2.

In 1999, testing began on 16 June using two levels of fog (none and full) and two levels of shade (40\% white shade cloth and none). Shade cloths were applied to both houses for a two day period, then removed for two days. During each two day period, fogging was permitted in alternate houses on alternate days. When permitted, fogging was employed only when air temperatures exceeded the evaporative pad set point by $0.5^{\circ} \mathrm{C}$ to avoid unnecessarily wetting the canopy and floor.

Table C1.2: Number of days each cooling treatment was imposed in the two houses during the summer of 1998.

\begin{tabular}{llll}
\hline Vent Rate & Shade & Mixing Fans & Days \\
\hline 1 & 0 & 0 & 13 \\
1 & 0 & 1 & 13 \\
\hline
\end{tabular}




\begin{tabular}{llll}
\hline 1 & 1 & 0 & 8 \\
1 & 1 & 1 & 8 \\
1 & 0 & 0 & 7 \\
1 & 1 & 0 & 7 \\
2 & 0 & 0 & 7 \\
2 & 1 & 0 & 7 \\
\hline
\end{tabular}

On 2 July, the fogging treatment was replaced by two levels of evaporative pad (full and none) and two levels of ventilation rate $\left(0.041 \mathrm{~m}^{3} \mathrm{~m}^{-2} \mathrm{~s}^{-1}\right.$ vs. $\left.0.087 \mathrm{~m}^{3} \mathrm{~m}^{-2} \mathrm{~s}^{-1}\right)$ in a series originally designed to span three eight-day sequences. The sequences were set up around shade cloth application. Because of the labor involved, shade was on for four days then off for four. Ventilation rates and evaporative pad cooling were applied on a two- or four-day cycle, depending upon the sequence. A detailed description of each sequence is presented below.

Sequence 1: A 40\% white shade cloth was applied to only one house for four days and then moved to the other for the next four. During each of those four-day periods, evaporative pad cooling was on in both houses for the first two days and off for the remaining two. During the evaporative pad cooling periods, the low ventilation rate was applied to both houses on one day and the high ventilation rate was applied on the other.

Sequence 2: Identical 40\% white shade cloths were applied to both houses for four days and then removed for four days. Evaporative cooling was on in both houses for the first two days of each four-day shade period and off for the remaining two days. During one of the two days the high ventilation rate was applied in one house and the low ventilation rate in the other. On the alternate day, the two ventilation rates were switched between the houses. Cloudy, cool weather conditions compromised this sequence so the data were disregarded and the sequence was repeated at the conclusion of the third sequence.

Sequence 3. Identical $40 \%$ white shade cloths were again applied to both houses for four days and removed for four. The high ventilation rate was used in both houses for two days of each four-day period and the low ventilation rate was used the other two days. For the first day of each two-day period, evaporative pad cooling was applied in one house but not the other. On the alternate day, application of evaporative pad cooling was reversed.

On 4 Aug, a third experiment was launched in which the white shade cloths were replaced with the 55\% black shade cloths used in 1998, except that the cloth was misted to dissipate the solar energy absorbed by the cloth. The treatment scheme was the same as described above for the July test, except that black shade plus misting was substituted for the dry white shade. It was not necessary to repeat any eight day sequence during this experiment so testing was completed in 24 days.

Finally, a follow-up fogging experiment was conducted during two days in October. The fogging nozzles were mounted as described above but the aspirated stations used for sensing temperature and relative humidity were rearranged to measure vertical gradients in the center of each house rather than horizontal gradients throughout the house. Both houses were empty of plants, but the bags the plants were grown in were left in place. One house was left as a control house in which neither the fans nor the nozzles were activated. Temperature setpoints were adjusted so that the same cooling equipment ran in both houses (ventilation rate $=0.041$ $\mathrm{m}^{3} \mathrm{~m}^{-2} \mathrm{~s}^{-1}$ and evaporative pad cooling on) throughout each test. 
Control of the test house was manipulated so that between 1030 and 1200 both fans and fog were activated, between 1200 and 1300 only the fans ran and between 1300 and 1400 only the fog ran. In all cases, the fans were running at high speed. The sequence was repeated during the afternoon but the duration of each treatment was reduced to one half-hour. A base-line comparison was conducted the following day in which neither fog nor fans ran in either house. 


\title{
C2 GREENHOUSE COOLING AS AFFECTED BY CANOPY DENSITY, VENTILATION RATE, EVAPORATIVE PAD COOLING, SHADE AND MIXING FANS
}

\author{
Dan H Willits \\ Biological and Agricultural Engineering, North Carolina State University
}

\section{Abstract}

A three-year study (1997 - 1999) was conducted to examine the effect of canopy density, ventilation rate, evaporative pad cooling, shade and mixing fans on the cooling of a greenhouse tomato crop. Ventilation rate was imposed at two levels $\left(0.041 \mathrm{~m}^{3} \mathrm{~m}^{-2} \mathrm{~s}^{-1} \mathrm{vs}\right.$. $0.087 \mathrm{~m}^{3} \mathrm{~m}^{-2} \mathrm{~s}^{-1}$ ), evaporative pad cooling at two levels (none and full), shade at three levels (50\% black, $40 \%$ white, and a misted, $50 \%$ black cloth), mixing fans at two levels (none and full). Canopy density was imposed at three levels, low density $\left(0.073\right.$ plants $\left.\mathrm{m}^{-2}\right)$, medium density (2.54 plants $\mathrm{m}^{-2}$ ) and high density (2.54 plants $\mathrm{m}^{-2}$ planted two months ahead of the medium density canopy). Higher canopy densities generally produced lower leaf temperatures, but in some cases the differences were not large. Shade was the most effective treatment for reducing leaf temperatures but not air temperatures. When used with evaporative pad cooling, the $40 \%$ white cloth was about as effective as the dry, $50 \%$ black cloth, but the misted, $50 \%$ black cloth was superior to either. When evaporative pad cooling was not used, there was no difference between the $40 \%$ white and the misted $50 \%$ black cloths. Evaporative pad cooling was the most effective treatment for reducing air temperature and the second most effective treatment for reducing leaf temperature. The high ventilation rate was not an effective cooling strategy when used without evaporative pad cooling, but it was very effective when evaporative pad cooling was used. Combining shade, evaporative pad cooling and high ventilation rate produced the lowest leaf temperatures observed in the study.

\section{Introduction}

A three-year study (1997 - 1999) was conducted to examine the effect of canopy density, ventilation rate, evaporative pad cooling, shade and mixing fans on the cooling of a greenhouse tomato crop. Ventilation rate was imposed at two levels $\left(0.041 \mathrm{~m}^{3} \mathrm{~m}^{-2} \mathrm{~s}^{-1} \mathrm{vs}\right.$. $0.087 \mathrm{~m}^{3} \mathrm{~m}^{-2} \mathrm{~s}^{-1}$ ) in all three years (but not in all tests). Evaporative pad cooling was imposed at two levels (none and full) in 1997 and 1999 and only one level (full) in 1998. Shade was imposed using a 50\% black polypropylene shade cloth in 1998 and in 1999 a $40 \%$ white cloth was used in the first test and a 50\% black cloth was used in the second, with the black cloth being misted. Mixing fans were imposed only in the July test of 1998. In 1997, one house (high density canopy) was planted at 2.54 plants $\mathrm{m}^{-2}$ on 1 May while the adjacent house (medium density canopy) was planted at 2.54 plants $\mathrm{m}^{-2}$ on 1 July. In 1998, one house (medium density canopy) was planted at 2.54 plants $\mathrm{m}^{-2}$ and the other (low density canopy) at 0.073 plants $\mathrm{m}^{-2}$, both on 16 June. In 1999, 2.54 plants $\mathrm{m}^{-2}$ were planted into both houses on 14 June. Air temperatures, relative humidities, leaf temperatures, structure temperatures, ground temperatures and solar radiation (inside and out) were measured as described in Chapter $\mathrm{C} 1$. The treatments and the manner in which they were imposed are also described there. 


\section{Analyses}

The data from the 3 years were analyzed using the GLM procedure of SAS (SAS, 1985). Treatment effects were included in the model as class variables in the general model

\section{VAR $=$ SOL TDBO RHOUT LAI VNT*SHD*PAD*FAN*DEN}

where VAR represents a generic dependent variable described below, SOL is solar radiation on a horizontal surface outside, TDBO is the outside dry bulb temperature, RHOUT is the outside relative humidity and LAI is the leaf area index based on total greenhouse floor area. VNT is the class variable for ventilation rate $(1=$ high, $0=$ low $)$, SHD is the class variable for shade $(0=$ none, $1=50 \%$ black, $2=40 \%$ white, $3=50 \%$ black misted $)$, PAD is the class variable for evaporative pad cooling $(0=$ none, $1=$ full $)$, FAN is the class variable for mixing fan operation $(0=$ none, $1=$ full $)$ and DEN is the class variable for canopy density $(1=$ low, 2 $=$ medium and $3=\mathrm{high}$ ). Independent variables that were not significant at the 0.05 level for a given dependent variable were not retained in the model for that variable. Where tests of canopy density were involved, LAI was eliminated from the model because of the confounding between LAI and DEN.

The LSMEANS option of the GLM procedure was used to produce least-square means by class, along with the probability that the means were different from each other and non-zero. The dependent variables tested were mean air and leaf temperatures at the air inlet, middle and the air exit locations along the axis of each house, and the rise of air and leaf temperatures from inlet to exit. Air temperature at the middle of the house was taken to be the average of the inlet and exit air temperatures because it was assumed to represent the overall air temperature at mid-house better than the air temperature measured at mid-canopy. Since only the hottest portions of the day were of interest, only periods where outside solar radiation was greater than $800 \mathrm{~W} \mathrm{~m}^{-2}$ were considered.

\section{Results}

\section{Canopy Density}

The effect of canopy density on greenhouse cooling was examined by considering three separate tests: 1) the 1997 test in which ventilation rate and evaporative pad cooling were varied for houses at two different canopy densities (medium and high); 2) the test conducted in July of 1998 where mixing fans were combined with 50\% black shade and two different canopy densities (low and medium); and 3) the test conducted in August of 1998 where ventilation rate and shade were combined with the same difference in canopy density (low and medium).

1997. Table C2.1 summarizes the outside environment and Table C2.2 presents the leastsquare means of air and leaf temperatures for 1997. LAI was not included in the model because of confounding between LAI and canopy density and the inability of GLM to separate the two effects. 
Table C2.3 presents canopy difference contrasts generated from Table C2.2. The general effect of increasing canopy density was to reduce air and leaf temperatures. Less reduction took place (and in some cases an overall increase occurred) when evaporative pad cooling was used compared to when it was not. It is possible that a point of diminishing returns was encountered with the higher humidities produced by the evaporative pad making the increased transpirational area available with the higher density canopy less effective, but it is at least as likely that differences in canopy height were involved, at least with respect to leaf temperatures.

Table C2.1: Means, standard deviations and ranges of outside solar radiation, dry bulb temperature and relative humidity observed during the 1997 tests.

\begin{tabular}{lllll}
\hline & Mean & Std Dev & Min & Max \\
\hline SOL $-\mathrm{W} \mathrm{m}^{-2}$ & 878 & 50 & 800 & 983 \\
TDBO $-{ }^{\circ} \mathrm{C}$ & 30.9 & 2.3 & 22.1 & 35.7 \\
RHOUT $-\%$ & 51.5 & 7.4 & 33.8 & 75.8 \\
\hline
\end{tabular}

Table C2.2: Medium vs. high density canopy effects from the 1997 study. Same letter superscripts for the same variable indicate no differences at $\alpha=0.05$. Differences between variables were not tested.

\begin{tabular}{lllllllllll}
\hline Vnt & Pad & Den & $\mathrm{Ta}_{\text {in }}$ & $\mathrm{Ta}_{\mathrm{md}}$ & $\mathrm{Ta}_{\mathrm{ex}}$ & $\mathrm{dTa}$ & $\mathrm{Tl}_{\text {in }}$ & $\mathrm{Tl}_{\mathrm{md}}$ & $\mathrm{Tl}_{\mathrm{ex}}$ & $\mathrm{dTl}$ \\
\hline 1 & 0 & 2 & $30.8^{\mathrm{b}}$ & $31.8^{\mathrm{a}}$ & 32.8 & 1.9 & $31.0^{\mathrm{a}}$ & $30.9^{\mathrm{b}}$ & 31.1 & 0.1 \\
1 & 0 & 3 & $31.5^{\mathrm{a}}$ & $31.6^{\mathrm{a}}$ & 31.6 & 0.1 & 30.0 & $29.6^{\mathrm{a}}$ & $30.4^{\mathrm{a}}$ & 0.4 \\
1 & 1 & 2 & 23.4 & $25.8^{\mathrm{b}}$ & $28.3^{\mathrm{a}}$ & 4.9 & $28.3^{\mathrm{b}}$ & $29.8^{\mathrm{a}}$ & $30.2^{\mathrm{a}}$ & 1.9 \\
1 & 1 & 3 & $23.9^{\mathrm{cd}}$ & $26.0^{\mathrm{b}}$ & $28.2^{\mathrm{a}}$ & 4.3 & $28.1^{\mathrm{b}}$ & $28.7^{\mathrm{c}}$ & $30.3^{\mathrm{a}}$ & 2.2 \\
2 & 0 & 2 & 30.3 & $30.7^{\mathrm{c}}$ & 31.0 & 0.7 & $30.8^{\mathrm{a}}$ & $30.9^{\mathrm{b}}$ & $30.4^{\mathrm{a}}$ & -0.4 \\
2 & 0 & 3 & $31.2^{\mathrm{ab}}$ & $30.6^{\mathrm{c}}$ & 30.0 & -1.2 & 29.0 & $29.0^{\mathrm{c}}$ & 29.6 & 0.6 \\
2 & 1 & 2 & $23.8^{\mathrm{d}}$ & 24.7 & $25.5^{\mathrm{b}}$ & 1.7 & $28.1^{\mathrm{b}}$ & $28.7^{\mathrm{c}}$ & 28.5 & 0.4 \\
2 & 1 & 3 & $24.2^{\mathrm{c}}$ & 25.0 & $25.8^{\mathrm{b}}$ & 1.6 & 26.6 & 27.1 & 28.2 & 1.5 \\
\hline
\end{tabular}

In the study reported in Chapter C3, the evaporative pad was seen to thermally stratify the air moving through the greenhouse at the lower ventilation rate such that lower temperature, higher humidity air coming off the pad moved near the floor while higher temperature, lower humidity air remained higher up. In this study, the velocity measurements somewhat confirm this, at least in the high density house. At the $0.6 \mathrm{~m}$ level, the velocity in the evaporatively cooled case was $80 \%$ greater than in the non-evaporatively cooled case, both at the lower ventilation rate. On the other hand, at the $2.1 \mathrm{~m}$ level, the velocity in the evaporatively cooled case was $65 \%$ that at the same level as when no evaporative pad cooling was used. Velocity differences with height decreased at the higher ventilation rate. In the evaporatively cooled case, velocity at the $0.6 \mathrm{~m}$ level was only $5 \%$ greater than in the non-evaporatively cooled case while at the $2.1 \mathrm{~m}$ level the velocity in the evaporatively cooled case was $90 \%$ of that in the non-evaporatively cooled case.

A similar trend was noted in the medium density house; however, differences in the velocity gradients at the lower ventilation rate were smaller and not statistically significant with respect to evaporative pad cooling. That is not to say the temperature gradients were not different. They were not measured in this study, nor were velocity gradients measured in the 
study reported in Chapter C3. Only a more thorough study linking velocity and temperature gradients is likely to give a complete answer.

In the high density canopy, the top was at the 2.0 to $2.2 \mathrm{~m}$ mark throughout testing (the plants were lowered as they grew). Leaf temperature measurements were always in the upper $0.3 \mathrm{~m}$ of each canopy, which would have placed them in the slowest moving, and probably the highest temperature, air during evaporative pad cooling at the lowest ventilation rate. Since the vertical velocity gradient differences imposed by evaporative pad cooling were smaller in the medium density canopy, this suggests that the leaf temperatures in the high-density canopy were higher relative to the medium density canopy than they would have been otherwise. This effect would have been mitigated at either the higher ventilation rate or in the non-evaporatively cooled case, which is reflected in the higher leaf temperature reductions evident for those cases in Table C2.2.

Table C2.3: Contrasts drawn from Table C2.2. Entries marked with an asterisk are not significantly different from zero at $\alpha=0.05$.

\begin{tabular}{lllllllllll}
\hline Vnt & Pad & Den & $\begin{array}{l}\mathrm{Ta}_{\text {in }} \\
\left({ }^{\circ} \mathrm{C}\right)\end{array}$ & $\begin{array}{l}\mathrm{Ta}_{\mathrm{md}} \\
\left({ }^{\circ} \mathrm{C}\right)\end{array}$ & $\begin{array}{l}\mathrm{Ta} \\
\left({ }^{\circ} \mathrm{C}\right)\end{array}$ & $\begin{array}{l}\mathrm{dTa} \\
\left({ }^{\circ} \mathrm{C}\right)\end{array}$ & $\begin{array}{l}\mathrm{Tl}_{\text {in }} \\
\left({ }^{\circ} \mathrm{C}\right)\end{array}$ & $\begin{array}{l}\mathrm{T} l_{\mathrm{md}} \\
\left({ }^{\circ} \mathrm{C}\right)\end{array}$ & $\begin{array}{l}\mathrm{Tl}_{\mathrm{ex}} \\
\left({ }^{\circ} \mathrm{C}\right)\end{array}$ & $\begin{array}{l}\mathrm{dTl} \\
\left({ }^{\circ} \mathrm{C}\right)\end{array}$ \\
\hline 1 & 0 & $2-3$ & -0.7 & $0.2^{*}$ & 1.1 & 1.8 & 1.0 & 1.3 & 0.7 & $-0.3^{*}$ \\
1 & 1 & $2-3$ & -0.5 & $-0.2^{*}$ & $0.1^{*}$ & 0.6 & $0.2^{*}$ & 1.0 & $-0.1^{*}$ & $-0.3^{*}$ \\
2 & 0 & $2-3$ & -0.8 & $0.1^{*}$ & 1.1 & 1.9 & 1.7 & 2.0 & 0.8 & -0.9 \\
2 & 1 & $2-3$ & -0.4 & -0.4 & $-0.3^{*}$ & $0.1^{*}$ & 1.4 & 1.6 & 0.4 & -1.1 \\
\hline
\end{tabular}

The results with respect to air temperature are not so easy to explain in the same fashion because the mid-house air temperatures presented here were means of the inlet and exhaust air temperatures and were assumed to represent the average air temperatures at mid-house. Consideration of the transpiration differences, however, suggests a possible explanation. Without evaporative pad cooling, and at the lower ventilation rate, transpiration per unit leaf area in the high density canopy (average LAI $=2.52$ ) was approximately $75 \%$ of that in the medium density canopy (average LAI $=1.14$ ). With evaporative pad cooling, transpiration in the high-density canopy was about $61 \%$ of that in the medium density canopy. With less evaporation taking place, it seems reasonable that the average air temperatures at mid-house might have been higher in the high density canopy when evaporative pad cooling was used.

July 1998. Table C2.4 summarizes the environmental data and Table C2.5 the air and leaf temperatures observed during the July 1998 test. The temperatures were averaged over mixing fan and shade cloth use because only small differences in canopy effects were observed in those treatments. The reductions in temperature afforded by the medium density canopy over the low-density canopy were small, averaging only about $0.25 \mathrm{C}$ for leaf temperature and about $0.5{ }^{\circ} \mathrm{C}$ for air temperature. The leaf temperature reductions were comparable with those in 1997 at the lower ventilation rate combined with evaporative pad cooling (the conditions used in the July 1998 test) but the air temperature reductions were greater. 
Table C2.4: Means, standard deviations and ranges of outside solar radiation, dry bulb temperature and relative humidity during July 1998 test.

\begin{tabular}{lllll}
\hline & Mean & Std Dev & Min & Max \\
\hline SOL $-\mathrm{W} \mathrm{m}^{-2}$ & 901 & 62 & 800 & 1115 \\
TDBO $-{ }^{\circ} \mathrm{C}$ & 30.5 & 2.2 & 24.4 & 35.6 \\
RHOUT $-\%$ & 48.6 & 9.9 & 31.4 & 88.8 \\
\hline
\end{tabular}

Table C2.5: Low vs. medium canopy density effects. The data were taken from the July 1998 test averaged over shade treatment and mixing fan operation. Same letter superscripts for the same variable indicate no differences at $\alpha=0.05$. Differences between variables were not tested.

\begin{tabular}{lllllllll}
\hline Den & $\begin{array}{l}\mathrm{Ta}_{\text {in }} \\
\left({ }^{\circ} \mathrm{C}\right)\end{array}$ & $\begin{array}{l}\mathrm{Ta}_{\mathrm{md}} \\
\left({ }^{\circ} \mathrm{C}\right)\end{array}$ & $\begin{array}{c}\mathrm{Ta}_{\mathrm{ex}} \\
\left({ }^{\circ} \mathrm{C}\right)\end{array}$ & $\begin{array}{l}\mathrm{dTa} \\
\left({ }^{\circ} \mathrm{C}\right)\end{array}$ & $\begin{array}{l}\mathrm{Tl}_{\text {in }} \\
\left({ }^{\circ} \mathrm{C}\right)\end{array}$ & $\begin{array}{l}\mathrm{Tl}_{\text {md }} \\
\left({ }^{\circ} \mathrm{C}\right)\end{array}$ & $\begin{array}{l}\mathrm{Tl}_{\text {ex }} \\
\left({ }^{\circ} \mathrm{C}\right)\end{array}$ & $\begin{array}{l}\mathrm{dTl} \\
\left({ }^{\circ} \mathrm{C}\right)\end{array}$ \\
\hline 1 & 23.6 & 26.1 & 28.8 & 5.2 & 27.9 & 28.7 & 29.4 & $1.5^{\mathrm{a}}$ \\
2 & 23.3 & 25.6 & 28.0 & 4.6 & 27.8 & 28.4 & 29.3 & $1.5^{\mathrm{a}}$ \\
\hline
\end{tabular}

August 1998. Table C2.6 presents the environmental data summarized for the August 1998 test and Table C2.7 presents the air and leaf temperatures for the same period. The reductions in air and leaf temperatures with increasing canopy density were greater in this test than in the July 1998 test, but were not consistently greater or less than the results from 1997. As a rule, air temperature rise from inlet to exit was reduced at the higher canopy density for all treatments. On the other hand, leaf temperature rise was increased at the lower ventilation rate (primarily by reducing leaf temperatures at the pad end of the house) but decreased by increasing canopy density at the higher ventilation rate.

Table C2.6: Means, standard deviations and ranges of outside solar radiation, dry bulb temperature and relative humidity observed during the August 1998 test.

\begin{tabular}{lllll}
\hline & Mean & Std Dev & Min & Max \\
\hline SOL $-\mathrm{W} \mathrm{m}^{-2}$ & 888 & 61 & 800 & 1115 \\
TDBO $-{ }^{\circ} \mathrm{C}$ & 30.7 & 2.0 & 24.4 & 35.6 \\
RHOUT $-\%$ & 49.3 & 9.4 & 31.5 & 88.8 \\
\hline
\end{tabular}

Table C2.8 presents contrasts drawn from the August 1998 test. The results suggest a fairly consistent effect on air temperature at both ventilation rates and shade levels; however, the effect on leaf temperature was less consistent. Canopy density reduced leaf temperatures less both at the higher ventilation rate and under shade.

Table C2.7: Low vs. medium density canopy effects as a function of ventilation rate and shade, both using evaporative pad cooling from the August 1998 test. 
Same letter superscripts for the same variable indicate no differences at $\alpha=0.05$. Differences between variables were not tested.

\begin{tabular}{lllllllllll}
\hline Vnt & Shd & Den & $\mathrm{Ta}_{\text {in }}$ & $\mathrm{Ta}_{\mathrm{md}}$ & $\mathrm{Ta}_{\mathrm{ex}}$ & $\mathrm{dTa}$ & $\mathrm{Tl}_{\mathrm{in}}$ & $\mathrm{Tl}_{\mathrm{md}}$ & $\mathrm{Tl}_{\mathrm{ex}}$ & $\mathrm{dTl}$ \\
\hline 1 & 0 & 1 & $24.9^{\mathrm{a}}$ & 27.5 & 30.1 & 5.2 & 31.3 & 32.7 & $31.1^{\mathrm{a}}$ & -0.2 \\
1 & 0 & 2 & $24.5^{\mathrm{b}}$ & 26.6 & $28.7^{\mathrm{a}}$ & $4.2^{\mathrm{a}}$ & 29.0 & 30.2 & $31.2^{\mathrm{a}}$ & $2.2^{\mathrm{a}}$ \\
1 & 1 & 1 & $24.7^{\mathrm{a}}$ & 26.8 & $28.8^{\mathrm{a}}$ & $4.1^{\mathrm{a}}$ & 28.7 & $29.4^{\mathrm{a}}$ & $29.5^{\mathrm{b}}$ & $0.8^{\mathrm{b}}$ \\
1 & 1 & 2 & $24.4^{\mathrm{d}}$ & $26.1^{\mathrm{a}}$ & 27.8 & 3.5 & $27.1^{\mathrm{a}}$ & $28.0^{\mathrm{b}}$ & 29.1 & $2.1^{\mathrm{a}}$ \\
2 & 0 & 1 & $25.0^{\mathrm{c}}$ & $26.2^{\mathrm{a}}$ & 27.3 & 2.3 & 28.4 & $29.4^{\mathrm{a}}$ & $29.4^{\mathrm{b}}$ & $0.9^{\mathrm{b}}$ \\
2 & 0 & 2 & $24.6^{\mathrm{b}}$ & 25.5 & 26.3 & $1.8^{\mathrm{a}}$ & 28.0 & $28.3^{\mathrm{c}}$ & $28.6^{\mathrm{c}}$ & $0.6^{\mathrm{c}}$ \\
2 & 1 & 1 & $24.9^{\mathrm{ac}}$ & 25.8 & 26.7 & $1.8^{\mathrm{a}}$ & $27.2^{\mathrm{a}}$ & $27.7^{\mathrm{b}}$ & $28.2^{\mathrm{c}}$ & $1.0^{\mathrm{b}}$ \\
2 & 1 & 2 & $24.4^{\mathrm{d}}$ & 25.1 & 25.9 & 1.5 & 26.7 & 26.8 & 27.3 & $0.6^{\mathrm{c}}$ \\
\hline
\end{tabular}

Table C2.8: Contrasts drawn from Table C2.7. Entries marked with an asterisk are not significantly different from zero at $\alpha=0.05$.

\begin{tabular}{lllllllllll}
\hline Vnt & Shd & Den & $\begin{array}{l}\mathrm{Ta} \text { in } \\
\left({ }^{\circ} \mathrm{C}\right)\end{array}$ & $\begin{array}{l}\mathrm{Tamd} \\
\left({ }^{\circ} \mathrm{C}\right)\end{array}$ & $\begin{array}{l}\mathrm{Ta} \text { ex } \\
\left({ }^{\circ} \mathrm{C}\right)\end{array}$ & $\begin{array}{l}\mathrm{dTa} \\
\left({ }^{\circ} \mathrm{C}\right)\end{array}$ & $\begin{array}{l}\mathrm{Tl}_{\text {in }} \\
\left({ }^{\circ} \mathrm{C}\right)\end{array}$ & $\begin{array}{l}\mathrm{Tl} \text { md } \\
\left({ }^{\circ} \mathrm{C}\right)\end{array}$ & $\begin{array}{l}\mathrm{T} l_{\text {ex }} \\
\left({ }^{\circ} \mathrm{C}\right)\end{array}$ & $\begin{array}{l}\mathrm{dTl} \\
\left({ }^{\circ} \mathrm{C}\right)\end{array}$ \\
\hline 1 & 0 & $1-2$ & 0.4 & 0.9 & 1.4 & 1.0 & 2.4 & 2.6 & $-0.1 *$ & -2.4 \\
1 & 1 & $1-2$ & 0.3 & 0.7 & 1.0 & 0.6 & 1.7 & 1.5 & 0.4 & -1.3 \\
2 & 0 & $1-2$ & 0.4 & 0.7 & 1.0 & 0.6 & 0.4 & 1.0 & 0.8 & 0.4 \\
2 & 1 & $1-2$ & 0.5 & 0.7 & 0.9 & 0.3 & 0.5 & 0.9 & 0.9 & 0.4 \\
\hline
\end{tabular}

The apparent discrepancy in the density effect between the two 1998 tests is probably the result of changes in radiation loading differences between canopies in the two tests. The July test was conducted when the plants in both houses were small and there was very little intracanopy or floor shading in either house. The long- and short-wave radiant loading per unit leaf area between canopies should have been more similar than during the August study, where the medium density canopy had closed but the low density canopy had not. In July, when no shade or mixing fans were used, the canopy in the low density house (average LAI = 0.11 ) transpired about $28 \%$ more per unit leaf area than the canopy in the medium density house (average LAI $=0.77$ ). In August, under the same conditions, the low density house (average LAI $=0.27$ ) transpired more than twice as much per unit leaf area as the medium density house (average LAI = 1.92). Since both studies were conducted with evaporative pad cooling (i.e., similar internal humidities), this suggests that during the July tests there was little difference in radiant loading per unit leaf area between the two canopies, resulting in a small canopy effect. In August, the radiant loading per unit leaf area was apparently much higher in the low density house, leading to a much larger canopy effect.

\section{Evaporative Pad Cooling vs. Ventilation Rate}

Evaporative pad cooling and ventilation rates are examined at a single canopy density (medium) using four datasets combined. Data was taken from: 1) the 1997 test in which ventilation rate and evaporative pad cooling were combined (only data from the medium density canopy were used); 2) the 1998 tests in which shade and evaporative pad cooling were combined with mixing fans in July and ventilation rate in August (again only the medium density data were used); and 3) the 1999 tests where ventilation rate and evaporative pad cooling were combined with two different shade types (both houses were at medium density). Data from days with mixing fans were eliminated from consideration. The 10 min readings 
from 1998 and 1999 were averaged over each half-hour to coincide with the data collection period of 1997. Table C2.9 summarizes the outside environment over the entire combined dataset.

Table C2.9: Means, standard deviations and ranges of outside solar radiation, dry bulb temperature and relative humidity.

\begin{tabular}{lllll}
\hline & Mean & Std Dev & Min & Max \\
\hline SOL $-\mathrm{W} \mathrm{m}^{-2}$ & 878 & 51 & 800 & 1029 \\
TDBO $-{ }^{\circ} \mathrm{C}$ & 31.6 & 2.6 & 25.0 & 38.9 \\
RHOUT $-\%$ & 47.8 & 10.7 & 24.6 & 83.4 \\
LAI $-\mathrm{m}^{2} \mathrm{~m}^{-2}$ & 1.19 & 0.51 & 0.28 & 2.33 \\
\hline
\end{tabular}

Table C2.10 presents the air and leaf temperatures for the dataset. SOL and LAI were not significant for $\mathrm{Ta}_{\mathrm{in}}$ and were therefore not included in the model for that variable. Since the effect of shade is treated separately below, including interactions with ventilation rate and evaporative pad cooling, only the data from the no shade case is presented at this time. As with the results presented in Table C2.2, leaf temperature was below air temperature when no evaporative pad cooling was used and higher than air temperature when it was. Without evaporative pad cooling, the separation between leaf and air temperatures diminished as ventilation rate increased. With evaporative pad cooling the separation stayed the same or diminished at the inlet and the middle, but increased at the exit. Leaf temperature gradients from inlet to exit were always less than those for air temperature, no matter the cooling mode or ventilation rate. Both air and leaf temperature gradients were larger with either evaporative pad cooling or low ventilation rate than with no evaporative pad cooling or high ventilation rate.

Table C2.10: Least-square means of air and leaf temperature at three locations (inlet, middle and exit) as a function of ventilation rate and evaporative pad cooling under no shade. Same letter superscripts for the same variable indicate no treatment differences at $\alpha=0.05$. Differences between variables were not tested.

\begin{tabular}{llllllllll}
\hline Vnt & $\mathrm{Pad}$ & $\begin{array}{c}\mathrm{Ta}_{\text {in }} \\
\left({ }^{\circ} \mathrm{C}\right)\end{array}$ & $\begin{array}{c}\mathrm{Ta} \text { md } \\
\left({ }^{\circ} \mathrm{C}\right)\end{array}$ & $\begin{array}{l}\mathrm{Ta} \text { ex } \\
\left({ }^{\circ} \mathrm{C}\right)\end{array}$ & $\begin{array}{l}\mathrm{dTa} \\
\left({ }^{\circ} \mathrm{C}\right)\end{array}$ & $\begin{array}{l}\mathrm{Tl} \text { in } \\
\left({ }^{\circ} \mathrm{C}\right)\end{array}$ & $\begin{array}{l}\mathrm{Tl} l_{\mathrm{md}} \\
\left({ }^{\circ} \mathrm{C}\right)\end{array}$ & $\begin{array}{l}\mathrm{Tl}_{\mathrm{ex}} \\
\left({ }^{\circ} \mathrm{C}\right)\end{array}$ & $\begin{array}{l}\mathrm{dTl} \\
\left({ }^{\circ} \mathrm{C}\right)\end{array}$ \\
\hline 1 & 0 & 31.8 & 32.6 & 33.4 & 1.7 & 31.5 & 31.8 & 31.7 & $0.2^{\mathrm{a}}$ \\
1 & 1 & $24.1^{\mathrm{a}}$ & 26.5 & 28.9 & 4.9 & 29.0 & 30.3 & 30.8 & 1.9 \\
2 & 0 & 31.4 & 31.6 & 31.8 & 0.4 & 31.1 & 31.2 & 31.2 & $0.0^{\mathrm{a}}$ \\
2 & 1 & $24.2^{\mathrm{a}}$ & 25.3 & 26.3 & 2.1 & 28.3 & 29.1 & 29.1 & 0.8 \\
\hline
\end{tabular}

Table C2.11 presents ventilation rate and evaporative pad cooling contrasts generated from Table C2.10. The results suggest that increased ventilation rate reduced temperatures more when evaporative pad cooling was used than when it was not. Without evaporative pad cooling, the effect of increased ventilation rate on leaf temperature was small (approximately $0.5^{\circ} \mathrm{C}$ ); however, the reduction was nearly uniform from end-to-end while that for air temperature was greater away from the pad. With evaporative pad cooling, the higher ventilation rate reduced leaf temperatures more at the exit end of the house. This suggests that the higher ventilation rate effectively extended the influence of the pad further down the length of the house. 
Table C2.11: Contrasts drawn from Table C2.10. Entries marked with an asterisk are not significantly different from zero at $\alpha=0.05$.

\begin{tabular}{llllllllll}
\hline Vnt & Pad & $\begin{array}{l}\text { Tain } \\
\left({ }^{\circ} \mathrm{C}\right)\end{array}$ & $\begin{array}{l}\mathrm{Ta}_{\mathrm{md}} \\
\left({ }^{\circ} \mathrm{C}\right)\end{array}$ & $\begin{array}{l}\mathrm{Ta} \\
\left({ }^{\circ} \mathrm{C}\right)\end{array}$ & $\begin{array}{l}\mathrm{dTa} \\
\left({ }^{\circ} \mathrm{C}\right)\end{array}$ & $\begin{array}{l}\mathrm{Tl}_{\text {in }} \\
\left({ }^{\circ} \mathrm{C}\right)\end{array}$ & $\begin{array}{l}\mathrm{Tl}_{\mathrm{md}} \\
\left({ }^{\circ} \mathrm{C}\right)\end{array}$ & $\begin{array}{l}\mathrm{Tl}_{\mathrm{ex}} \\
\left({ }^{\circ} \mathrm{C}\right)\end{array}$ & $\begin{array}{l}\mathrm{dTl} \\
\left({ }^{\circ} \mathrm{C}\right)\end{array}$ \\
\hline $1-2$ & 0 & 0.4 & 1.0 & 1.6 & 1.3 & 0.3 & 0.6 & 0.5 & $0.2^{*}$ \\
$1-2$ & 1 & $-0.2^{*}$ & 1.3 & 2.7 & 2.8 & 0.7 & 1.2 & 1.8 & 1.1 \\
1 & $0-1$ & 7.7 & 6.1 & 4.5 & -3.2 & 2.5 & 1.5 & 0.9 & -1.7 \\
2 & $0-1$ & 7.2 & 6.4 & 5.5 & -1.7 & 2.8 & 2.1 & 2.1 & -0.7 \\
\hline
\end{tabular}

The evaporative pad cooling contrasts show that the pad reduced air temperatures more than leaf temperatures, and the reduction was greater nearer the pad, as might be expected, at least for the low ventilation rate. At the higher ventilation rate, leaf temperatures were reduced nearly uniformly from end-to-end. As with the previous contrasts, the evaporative pad contrasts suggest that evaporative pad cooling was enhanced by the higher ventilation rate.

\section{Shade}

The effect of shading on cooling was examined by considering data from four tests, all at the medium canopy density. Data from the July 1998 test (excluding that from days on which mixing fans ran in either house) were combined with the data from the ventilation rate and shade test conducted in August 1998 and the ventilation rate, shade and evaporative pad cooling tests conducted in 1999. Table C2.12 summarizes the environmental variables and Table C2.13 presents the air and leaf temperatures. The dry 50\% black shade cloth $($ Shd $=1)$ reduced air temperature gradient by about $22.4 \%$, primarily by reducing air temperatures away from the pad. This is less than the reduction that would be predicted by the shade rating of the cloth $(50 \%)$ but is consistent with air temperature reductions found in other studies (Willits and Peet, 1993). Leaf temperatures were reduced more uniformly from end-to-end, which resulted in little reduction in the gradient. In general, the $40 \%$ white shade cloth (Shd $=$ 2) performed about the same as the dry $50 \%$ black cloth, while the $50 \%$ misted shade cloth $($ Shd $=3)$ provided additional leaf temperature reduction but not always additional air temperature reduction.

Table C2.12: Means, standard deviations and ranges of outside solar radiation, dry bulb temperature, relative humidity and LAI.

\begin{tabular}{lllll}
\hline & Mean & Std Dev & Min & Max \\
\hline SOL $-\mathrm{W} \mathrm{m}^{-2}$ & 872 & 52 & 800 & 1035 \\
TDBO $-{ }^{\circ} \mathrm{C}$ & 32.2 & 2.5 & 25.4 & 38.9 \\
RHOUT - \% & 46.7 & 11.3 & 25.6 & 89.1 \\
LAI $-\mathrm{m}^{2} \mathrm{~m}^{-2}$ & 1.28 & 0.55 & 0.28 & 2.36 \\
\hline
\end{tabular}

Table C2.13: Least-square means of air and leaf temperature as a function of shade and ventilation rate for evaporative pad cooling. Same letter superscripts for the same variable indicate no treatment differences at $\alpha=0.05$. Differences between variables were not tested.

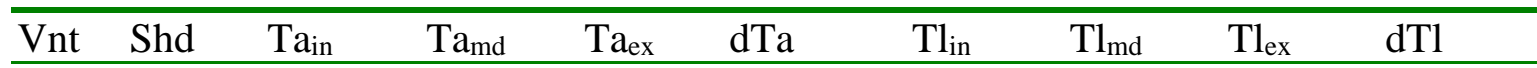




\begin{tabular}{|c|c|c|c|c|c|c|c|c|c|}
\hline & & $\left({ }^{\circ} \mathrm{C}\right)$ & $\left({ }^{\circ} \mathrm{C}\right)$ & $\left({ }^{\circ} \mathrm{C}\right)$ & $\left({ }^{\circ} \mathrm{C}\right)$ & $\left({ }^{\circ} \mathrm{C}\right)$ & $\left({ }^{\circ} \mathrm{C}\right)$ & $\left({ }^{\circ} \mathrm{C}\right)$ & $\left({ }^{\circ} \mathrm{C}\right)$ \\
\hline 1 & 0 & $24.6^{\mathrm{a}}$ & 27.1 & 29.5 & 4.9 & 29.5 & 30.8 & 31.4 & $1.9^{\mathrm{a}}$ \\
\hline 1 & 1 & $24.3^{b}$ & $26.2^{\mathrm{a}}$ & 28.2 & 3.8 & $27.4^{\mathrm{a}}$ & $28.3^{\mathrm{a}}$ & $29.2^{\mathrm{a}}$ & $1.7^{\mathrm{a}}$ \\
\hline 1 & 2 & $24.4^{\mathrm{b}}$ & $26.0^{\mathrm{b}}$ & $27.7^{\mathrm{a}}$ & 3.3 & $27.3^{\mathrm{ab}}$ & $28.6^{\mathrm{a}}$ & $29.1^{\mathrm{a}}$ & $1.7^{\mathrm{a}}$ \\
\hline 1 & 3 & $24.5^{\mathrm{ab}}$ & $26.0^{\mathrm{ab}}$ & $27.5^{\mathrm{a}}$ & 2.9 & $27.0^{\mathrm{bc}}$ & $27.5^{b}$ & $28.1^{\mathrm{b}}$ & $1.1^{\mathrm{b}}$ \\
\hline 2 & 0 & $24.7^{\mathrm{a}}$ & 25.8 & 26.8 & $2.1^{\mathrm{a}}$ & 28.5 & 29.3 & 29.4 & $0.9^{\mathrm{b}}$ \\
\hline 2 & 1 & $24.4^{\mathrm{ab}}$ & $25.5^{\mathrm{c}}$ & 26.5 & $2.0^{\mathrm{a}}$ & $27.2^{\mathrm{ab}}$ & $27.3^{b}$ & $27.6^{\mathrm{c}}$ & 0.4 \\
\hline 2 & 2 & $24.7^{\mathrm{ac}}$ & $25.3^{\mathrm{c}}$ & $25.9^{\mathrm{b}}$ & $1.3^{\mathrm{b}}$ & $26.7^{\mathrm{c}}$ & $27.8^{b}$ & $27.9^{b c}$ & $1.2^{\mathrm{b}}$ \\
\hline 2 & 3 & $24.7^{\mathrm{ac}}$ & $25.4^{\mathrm{c}}$ & $26.1^{b}$ & $1.4^{\mathrm{b}}$ & 26.1 & 26.6 & 27.1 & $1.1^{\mathrm{b}}$ \\
\hline
\end{tabular}

Table C2.14 presents contrasts between shade types at the lower ventilation rate. The results suggest that all forms of shading reduced leaf temperatures more than air temperatures. This seems reasonable since shading would directly affect leaf temperature by reducing the solar loading on the leaf while the effect on air temperature would be more indirect through energy exchange with an irradiated surface. White shade (0-3) reduced $\mathrm{Ta}_{\mathrm{in}}$ and $\mathrm{Ta}_{\mathrm{md}}$ more than dry black shade (0-1), but it was no better in reducing leaf temperatures. On the other hand, the misted shade was better at reducing leaf temperatures than either of the other two shade treatments. It was not better, however, at reducing air temperatures.

Table C2.14: Contrasts drawn from Table C2.13 comparing the three shade treatments at the low ventilation rate with evaporative pad cooling. Entries marked with an asterisk are not significantly different from zero at $\alpha=0.05$.

\begin{tabular}{llllllllll}
\hline Vnt & Shd & $\begin{array}{l}\mathrm{Ta}_{\text {in }} \\
\left({ }^{\circ} \mathrm{C}\right)\end{array}$ & $\begin{array}{l}\mathrm{Ta}_{\mathrm{md}} \\
\left({ }^{\circ} \mathrm{C}\right)\end{array}$ & $\begin{array}{l}\mathrm{Ta}_{\mathrm{ex}} \\
\left({ }^{\circ} \mathrm{C}\right)\end{array}$ & $\begin{array}{l}\mathrm{dTa} \\
\left({ }^{\circ} \mathrm{C}\right)\end{array}$ & $\begin{array}{l}\mathrm{Tl}_{\text {in }} \\
\left({ }^{\circ} \mathrm{C}\right)\end{array}$ & $\begin{array}{l}\mathrm{Tl}_{\mathrm{md}} \\
\left({ }^{\circ} \mathrm{C}\right)\end{array}$ & $\begin{array}{l}\mathrm{Tl}_{\mathrm{ex}} \\
\left({ }^{\circ} \mathrm{C}\right)\end{array}$ & $\begin{array}{l}\mathrm{dTl} \\
\left({ }^{\circ} \mathrm{C}\right)\end{array}$ \\
\hline 1 & $0-1$ & 0.3 & 0.9 & 1.4 & 1.1 & 2.1 & 2.5 & 2.2 & $0.1^{*}$ \\
1 & $0-2$ & 0.2 & 1.1 & 1.8 & 1.6 & 2.2 & 2.2 & 2.3 & $0.1^{*}$ \\
1 & $0-3$ & $0.1^{*}$ & 1.1 & 2.0 & 1.9 & 2.5 & 3.3 & 3.3 & 0.8 \\
\hline
\end{tabular}

Overall, the cooling provided by the white shade cloth was approximately the same as that provided by the dry black cloth. This may be because the black cloth would have absorbed more solar energy than the white (based upon color differences), some of which would have inevitably been transferred into the greenhouse. On the other hand, the white cloth would have allowed more direct solar energy gain by the house based on its shade rating. The results suggest that these effects were approximately equal, although it is not immediately clear why the white shade reduced mid-house and exit air temperatures more than the black. The wet $50 \%$ black shade (0-3) reduced leaf temperatures more than either of the other two shading treatments, probably because the energy normally trapped by the black cloth (or transmitted by the white cloth) would have been released to the atmosphere through the evaporation of water rather than transferred into the greenhouse.

Table C2.15 presents the shading treatments contrasted at the higher ventilation rate. The reduction in leaf temperatures resulting from adding shade at the higher ventilation rate was about $2 / 3$ or better than that at the lower ventilation rate. This suggests that the effect of adding shade and increasing ventilation rate is partially cumulative. The reduction in air temperatures in Table C2.15, however, was much less $(1 / 2$ or less of that shown in Table C2.14) which suggests that combining shade and increased ventilation rate is less effective in reducing air temperature. 
Table C2.15: Contrasts drawn from Table C2.13 comparing the three shade treatments at the higher ventilation rate with evaporative pad cooling. Entries marked with an asterisk are not significantly different from zero at $\alpha=0.05$.

\begin{tabular}{llllllllll}
\hline Vnt & Shd & $\begin{array}{l}\mathrm{Ta}_{\text {in }} \\
\left({ }^{\circ} \mathrm{C}\right)\end{array}$ & $\begin{array}{l}\mathrm{Ta} \text { md } \\
\left({ }^{\circ} \mathrm{C}\right)\end{array}$ & $\begin{array}{l}\mathrm{Ta}_{\mathrm{ex}} \\
\left({ }^{\circ} \mathrm{C}\right)\end{array}$ & $\begin{array}{l}\mathrm{dTa} \\
\left({ }^{\circ} \mathrm{C}\right)\end{array}$ & $\begin{array}{l}\mathrm{Tl}_{\text {in }} \\
\left({ }^{\circ} \mathrm{C}\right)\end{array}$ & $\begin{array}{l}\mathrm{Tl}_{\mathrm{md}} \\
\left({ }^{\circ} \mathrm{C}\right)\end{array}$ & $\begin{array}{l}\mathrm{Tl}_{\mathrm{ex}} \\
\left({ }^{\circ} \mathrm{C}\right)\end{array}$ & $\begin{array}{l}\mathrm{dTl} \\
\left({ }^{\circ} \mathrm{C}\right)\end{array}$ \\
\hline 2 & $0-1$ & 0.3 & 0.3 & 0.3 & $0.0^{*}$ & 1.3 & 2.1 & 1.8 & 0.5 \\
2 & $0-2$ & $0.0^{*}$ & 0.5 & 0.9 & 0.9 & 1.8 & 1.6 & 1.5 & -0.3 \\
2 & $0-3$ & $0.1^{*}$ & 0.4 & 0.7 & 0.6 & 2.4 & 2.8 & 2.3 & $-0.1^{*}$ \\
\hline
\end{tabular}

The data suggest that, under conditions of evaporative pad cooling, shading is superior to increased ventilation rate for reducing leaf temperatures but inferior in reducing air temperatures. It must be remembered, however, that shading reduces the amount of light available to the canopy, potentially reducing photosynthesis. If the canopy is not at or near light saturation, yields could be substantially reduced. On the other hand, increasing ventilation rate is generally more expensive, both in initial and operational costs.

Table C2.16: Means, standard deviations and ranges of outside solar radiation, dry bulb temperature, relative humidity and LAI.

\begin{tabular}{lllll}
\hline & Mean & Std Dev & Min & Max \\
\hline SOL $-\mathrm{W} \mathrm{m}{ }^{-2}$ & 871 & 47 & 800 & 1035 \\
TDBO $-{ }^{\circ} \mathrm{C}$ & 32.5 & 2.5 & 25.0 & 38.9 \\
RHOUT $-\%$ & 45.6 & 12.3 & 24.6 & 89.1 \\
LAI $-\mathrm{m}^{2} \mathrm{~m}^{-2}$ & 1.23 & 0.43 & 0.46 & 2.18 \\
\hline
\end{tabular}

To compare shade at both levels of evaporative cooling and ventilation rate, only the two studies from 1999 were considered. Table C2.16 summarizes the environment and Table $\mathrm{C} 2.17$ the air and leaf temperatures. There was no difference in the shade effect at the two ventilation rates so the data presented are for the low rate only. The reduction of $\mathrm{Ta}_{\mathrm{ex}}$ under the white shade cloth was not as large as when evaporative pad cooling was used $\left(1.2{ }^{\circ} \mathrm{C}\right.$ vs. $\left.1.8^{\circ} \mathrm{C}\right)$ but the effect on leaf temperatures was about the same $\left(1.9^{\circ} \mathrm{C}\right.$ vs. $2.2{ }^{\circ} \mathrm{C}$ for $\mathrm{Tl}_{\text {in }}$ and $1.8{ }^{\circ} \mathrm{C}$ vs. $2.3{ }^{\circ} \mathrm{C}$ for $\mathrm{Tl}_{\mathrm{ex}}$ ). Although the wet $50 \%$ black cloth produced air and leaf temperatures that were marginally lower than the $40 \%$ white, the differences were not significant at the 0.05 level. This suggests that the lower humidities associated with the lack of evaporative pad cooling may have allowed the canopy to compensate for the increased solar loading under the white cloth, whereas in the evaporatively cooled case it could not because of reduced transpiration.

Table C2.17: Least-square means of air and leaf temperature as a function of shade for low ventilation rate and no evaporative pad cooling. Same letter superscripts for the same variable indicate no treatment differences at $\alpha=0.05$. Differences between variables were not tested.

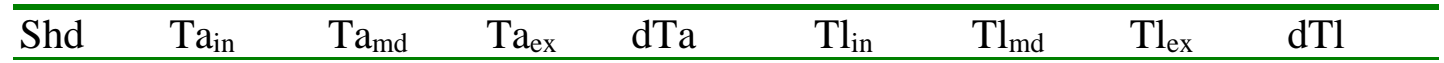




\begin{tabular}{lllllllll}
\hline & $\left({ }^{\circ} \mathrm{C}\right)$ & $\left({ }^{\circ} \mathrm{C}\right)$ & $\left({ }^{\circ} \mathrm{C}\right)$ & $\left({ }^{\circ} \mathrm{C}\right)$ & $\left({ }^{\circ} \mathrm{C}\right)$ & $\left({ }^{\circ} \mathrm{C}\right)$ & $\left({ }^{\circ} \mathrm{C}\right)$ & $\left({ }^{\circ} \mathrm{C}\right)$ \\
\hline 0 & $32.7^{\mathrm{a}}$ & $33.4^{\mathrm{a}}$ & 34.1 & 1.4 & 32.0 & 32.4 & 32.2 & $0.2 \mathrm{a}$ \\
2 & $32.7^{\mathrm{a}}$ & $32.8^{\mathrm{b}}$ & $32.9^{\mathrm{a}}$ & 0.2 & $30.1^{\mathrm{a}}$ & $30.1^{\mathrm{a}}$ & $30.4^{\mathrm{a}}$ & $0.2 \mathrm{a}$ \\
3 & $33.3^{\mathrm{a}}$ & $32.9^{\mathrm{ab}}$ & $32.4^{\mathrm{a}}$ & -0.9 & $29.8^{\mathrm{a}}$ & $29.8^{\mathrm{a}}$ & $30.3^{\mathrm{a}}$ & 0.5 \\
\hline
\end{tabular}

\section{Mixing Fans}

The effect of mixing fans on cooling was examined by considering data from a single test conducted in July of 1998, where mixing fans, evaporative pad cooling and the $50 \%$ shade cloth were combined with the low and medium density canopies. Table C2.18 presents the air and leaf temperatures for the test while the environmental summary is the same as that shown in Table C2.4. There were essentially no differences in the effect of the mixing fans on either canopy density or shade treatment; thus the data presented are averages over shade and canopy density.

Table C2.18: The effect of mixing fans on air and leaf temperatures. Data are averaged over shade treatment and canopy density. Same letter superscripts for the same variable indicate no differences at $\alpha=0.05$. Differences between variables were not tested.

\begin{tabular}{lllllllll}
\hline Fan & $\begin{array}{l}\text { Tain } \\
\left({ }^{\circ} \mathrm{C}\right)\end{array}$ & $\begin{array}{l}\mathrm{Ta} \text { md } \\
\left({ }^{\circ} \mathrm{C}\right)\end{array}$ & $\begin{array}{l}\mathrm{Ta}_{\text {ex }} \\
\left({ }^{\circ} \mathrm{C}\right)\end{array}$ & $\begin{array}{l}\mathrm{dTa} \\
\left({ }^{\circ} \mathrm{C}\right)\end{array}$ & $\begin{array}{l}\mathrm{Tl}_{\text {in }} \\
\left({ }^{\circ} \mathrm{C}\right)\end{array}$ & $\begin{array}{l}\mathrm{Tl} \text { md } \\
\left({ }^{\circ} \mathrm{C}\right)\end{array}$ & $\begin{array}{l}\mathrm{Tl}_{\text {ex }} \\
\left({ }^{\circ} \mathrm{C}\right)\end{array}$ & $\begin{array}{l}\mathrm{dTl} \\
\left({ }^{\circ} \mathrm{C}\right)\end{array}$ \\
\hline 0 & $23.5^{\mathrm{a}}$ & 25.9 & $28.4^{\mathrm{a}}$ & $4.9^{\mathrm{a}}$ & 28.1 & 29.8 & 29.7 & 1.7 \\
1 & $23.5^{\mathrm{a}}$ & 25.8 & $28.4^{\mathrm{a}}$ & $4.9^{\mathrm{a}}$ & 27.6 & 28.2 & 29.0 & 1.3 \\
\hline
\end{tabular}

The data show essentially no reduction in air temperature and only a small reduction in leaf temperature (approximately $0.6^{\circ} \mathrm{C}$ ) resulting from the use of mixing fans, which suggests that mixing fans are a relatively ineffective means of cooling a tomato canopy. This is in agreement with the conclusions reached in Chapters T4 and C2 where mixing fans were used in similar, but slightly different situations from the one reported here.

There is some evidence that these results may not be conclusive. In the section above on canopy density effects and in Chapter $\mathrm{C} 3$ of this report, evidence was presented of thermal stratification resulting from the use of the evaporative pad at the lower ventilation rate. The data presented in Chapter C3 suggest that this type thermal stratification is beneficial when the plants are relatively small, as they were during this mixing fan test, in that lower temperature air moves near the floor while higher temperature air remains at the upper levels of the greenhouse. Since this test was also conducted with evaporative pad cooling and at the lower ventilation rate, it is possible that the mixing fans were not as influential as they might have been at a later stage of growth when the upper portion of the canopy reached the slower moving, warmer air.

\section{Summary and Conclusions}

The effect of canopy density on air and leaf temperatures was not consistent over all cooling treatments. Although leaf temperatures were generally reduced in the high density canopy (compared to the medium density canopy), air temperatures were typically increased when 
evaporative pad cooling was used and only marginally reduced when it was not. On the other hand, air temperatures in the medium density canopy were reduced approximately the same as leaf temperatures compared to the low density canopy. The evidence suggests two factors that contributed to the differences observed. Relative to the low vs. medium density canopy, the differences appeared to be influenced by differences in intra-canopy shading which almost certainly led to differences in radiant loading per unit leaf surface area, and therefore different leaf temperatures.

On the other hand, the differences noted between the high and medium density canopies seemed to be related to canopy height and the interaction between it and thermal stratification of the air stream. The data suggest that the leaf temperatures measured in the high-density canopy were in the slowest moving, and perhaps highest temperature, air when evaporative pad cooling was combined with the lower ventilation rate. For either the higher ventilation rate or the non-evaporatively cooled case, vertical velocity gradients were significantly reduced and the higher canopy density produced significantly lower leaf temperatures. The marginal or negative reduction of air temperatures in the high density canopy when evaporative pad cooling was used seems to be the result of diminished transpiration in the high density canopy, perhaps because of reduced air velocities in the upper level of that canopy.

Compared to the low ventilation rate with no evaporative pad cooling, the most effective single means for reducing leaf temperatures of those tested here was the application of $40 \%$ white shade, with a mean reduction of about $2{ }^{\circ} \mathrm{C}$, followed by evaporative pad cooling, with a mean reduction of about $1.6{ }^{\circ} \mathrm{C}$. The misted, $50 \%$ black shade did not reduce leaf temperatures significantly more than the $40 \%$ white shade when evaporative pad cooling was not used. The dry, $50 \%$ black shade was not tested without evaporative pad cooling, but with evaporative pad cooling it did not perform significantly different from the $40 \%$ white. Increasing ventilation rate did not significantly reduce leaf temperatures when evaporative pad cooling was not used. Shading reduced air temperatures very little whereas evaporative pad cooling reduced air temperatures by more than $6{ }^{\circ} \mathrm{C}$.

Combining treatments was especially productive in some cases. Combining the high ventilation rate with evaporative pad cooling reduced leaf temperatures by about $2.8{ }^{\circ} \mathrm{C}$. On the other hand, combining $40 \%$ white shade with evaporative pad cooling reduced leaf temperatures by about $3.9^{\circ} \mathrm{C}$. The addition of shade to evaporative pad cooling reduced air temperature about as much as increasing ventilation rate, approximately $1{ }^{\circ} \mathrm{C}$. Combining shade with the high ventilation rate did not produce additional benefits without evaporative pad cooling; however, when all three treatments were combined the mean reduction in leaf temperature was approximately $4.8^{\circ} \mathrm{C}$.

The dry 50\% black cloth and the dry $40 \%$ white cloth reduced leaf temperatures approximately the same. The misted, 50\% black cloth cooled better than either dry cloth when evaporative pad cooling was used, suggesting that water evaporation from the cloth released absorbed solar energy to the atmosphere that would have otherwise been transferred into the greenhouse. Without evaporative pad cooling, however, there was little difference in the performance of the misted $50 \%$ black and the dry $40 \%$ white.

Shading reduced leaf temperatures more than evaporative pad cooling or increased ventilation rate; however, the choice between them cannot depend upon leaf temperature reduction alone. The potential for photosynthesis reduction must also considered. Typical mid-day levels of photosynthetically active radiation (PAR) at the top of the canopy in the summer in Raleigh range from about 900 to $1100 \mu \mathrm{mol} \mathrm{m} \mathrm{m}^{-2} \mathrm{~s}^{-1}$, depending upon cloud cover and haze. The levels 
of shade considered in this study would have reduced that to about 540 to $660 \mu \mathrm{mol} \mathrm{m} \mathrm{m}^{-2} \mathrm{~s}^{-1}$ under the $40 \%$ white cloth and 450 to 550 under the $50 \%$ black cloth. These levels are very close to the saturation points typically reported for the individual leaves of most crops.

Mastalerz (1977), for example, suggests that single leaves from a high light plant saturate at approximately 2000 to $3000 \mathrm{ft}-\mathrm{c}$ (approximately $400-600 \mu \mathrm{mol} \mathrm{m}^{-2} \mathrm{~s}^{-1}$ ). If whole canopy photosynthesis is considered, saturation levels could be as much as 10 to $50 \%$ higher, depending upon canopy density (Hanan et al., 1978). The amount of photosynthetic reduction will also depend upon the time required to deploy and retract the shade. For semi-permanent installations, such as externally mounted shade cloths, there would be little opportunity to deploy or retract in response to changing light levels, and therefore some compromise would need to be reached relative to photosynthesis reduction vs. temperature reduction. For internally mounted, automatically controlled shade cloths, some manipulation of the cloth in response to light would be possible, but the time lags usually required for full deployment would still require some compromise between cooling and productivity.

Whether or not shade induced reduction of photosynthetic activity would prove to be economically important in a high light crop such as tomato is a question that will have to be left to further study. On the other hand, it seems certain that since the saturation level for shade plants is only about 1/3 of that for high light plants (Mastalerz, 1977), shade would likely be the first choice for low light plants. Nevertheless, if a single cooling alternative is insufficient, as it often will be in high light, high temperature areas, the results of this study suggest that combinations of shading, evaporative cooling and increased ventilation rate can be used to significant advantage.

Finally, mixing fans used to increase local canopy air velocities did not prove to be effective in these tests under the conditions in which they were applied. As indicated in the discussion, the results might have been affected by the fact that the relatively young plants on which they were used were already immersed in the coolest air coming off the pad which might not have been the most effective environment in which to use mixing fans.

\section{Nomenclature}

$\begin{array}{ll}\text { Symbol } & \text { Description } \\ \text { DEN } & \text { canopy density class variable }(1=\text { low, } 2=\text { medium, } 3=\text { high }), \\ \text { air temperature gain from inlet to exit, }{ }^{\circ} \mathrm{C}, \\ \text { dTl } & \text { leaf temperature gain from inlet to exit, }{ }^{\circ} \mathrm{C}, \\ \text { FAN } & \text { mixing fan class variable }(0=\text { off, } 1=\text { on }), \\ \text { LAI } & \text { leaf area index based on total greenhouse area, } \mathrm{m}^{2} \mathrm{~m}^{-2}, \\ \text { PAD } & \text { evaporative pad cooling class variable }(0=\text { off, } 1=0 \text { on }), \\ \text { RHOUT } & \text { outside relative humidity, } \%, \\ \text { SOL } \quad \text { outside solar radiation on a horizontal surface, } \mathrm{W} \mathrm{m}^{-2}, & \text { shade class variable }(1=50 \% \text { black, } 2=40 \% \text { white, } 3=\text { misted } 50 \% \text { black }), \\ \text { SHD } & \text { air temperature, }{ }^{\circ} \mathrm{C}, \\ \text { Ta } & \text { outside dry bulb temperature, }{ }^{\circ} \mathrm{C}, \\ \text { TDBO } & \text { leaf temperature, },{ }^{\circ} \mathrm{C},\end{array}$


VAR a general independent variable used in EQ 1,

VNT ventilation rate class variable (1=low, 2=high),

Subscript $\quad$ Description

in inlet,

md middle

ex exit.

\section{References}

Hanan, J.J., Holley, W.D. and Goldsberry, K.L. 1978. Greenhouse management. SpringerVerlag, New York. pp. 530.

Mastalerz, John W. 1977. The greenhouse environment. John Wiley \& Sons, New York. pp. 629.

Willits, D.H. and Peet, M.M. 1993. The effect of evaporative cooling o the efficiency of external greenhouse shade cloths. Paper no. 93-4042 presented to the summer meeting of the ASAE, Spokane Center, Spokane, WA. 


\title{
C3 COMBINING FOG AND MIXING FANS WITH EVAPORATIVE PAD COOLING
}

\author{
Dan H Willits \\ Biological and Agricultural Engineering, North Carolina State University
}

\section{Abstract}

The effect of adding fog to evaporative pad cooling was examined to see if the heat stress observed in young transplants in previous years could be relieved. In the summer tests, temperatures at canopy level were seen to increase, rather than decrease, when the fogging system operated. Further investigation suggested that the mixing fans used to keep the fog droplets suspended were inverting the thermal stratification imposed by the evaporative pad. Subsequent testing conducted at the end of the summer provided further evidence to that effect. The results also suggested that careful consideration of the location and use of the mixing fans will be necessary if fogging is to be a viable cooling alternative for young plants.

\section{Introduction}

Observations in the 1997 and 1998 studies suggested that young transplants might be experiencing heat stress even when evaporative pad cooling was used. In 1997, during the first week after transplant the leaves in the medium density canopy were slightly paler than those in the high density canopy, where temperatures were lower and humidities were higher. In 1998, again during the first week after transplant, the leaves in the low density canopy were even paler than those in the medium density canopy. It was assumed that this was a consequence of either the higher leaf temperatures (Chapter C2) or the higher transpiration per unit leaf area (Chapter A2) observed in the lower density canopy. To test this, a fogging test was implemented in 1999 (16 June - 2 July) to artificially add moisture to the greenhouse air during the first two weeks after transplant to see if that would compensate for the limited transpiration capacity of the young canopy.

The plants were newly transplanted on 16 June. Cooling treatments consisted of two $40 \%$ white shade cloths applied to both houses on a four-day cycle, on for two days then off for two. During each two-day period, fogging was permitted in alternate houses on alternate days if the temperature was sufficiently high. Mixing fans mounted behind each fogging nozzle were used to keep the fog droplets suspended to promote evaporation and to minimize floor and canopy wetting. Evaporative cooling was used in both houses and ventilation rates were set to $0.041 \mathrm{~m}^{3} \mathrm{~m}^{-2} \mathrm{~s}^{-1}$. Additional details of the experimental schedule and set-up are outlined in Chapter C1.

The compromised by cool, cloudy weather such These short term tests consisted of fogging one of the two houses, with and without the mixing fans running, while leaving the adjacent house as a control. The results showed that whenever the fog and fans were both operating, air and leaf temperatures at canopy level $(0.6 \mathrm{~m})$ increased by as much as $2{ }^{\circ} \mathrm{C}$ in the fogged house compared to the unfogged house. When the fans were turned off and only the fogging nozzles operated, air and leaf temperatures in the fogged house dropped by as much as $4{ }^{\circ} \mathrm{C}$ (i.e., $2{ }^{\circ} \mathrm{C}$ cooler below the unfogged house).

The original experimental design was compromised by cool, cloudy weather such that short term tests were implemented to take a closer look at an unexpected trend that surfaced during 
regular testing. Whenever the fogging system operated, air temperatures at canopy level rose by several degrees. Since this was not expected, some short term tests were implemented to see what might be behind this. It seemed to be related to the operation of the mixing fans, so tests were devised such that one of the two houses was fogged, with and without the mixing fans running, while the adjacent house remained unfogged. The data collected showed that whenever the fog and fans were both on, air and leaf temperatures at canopy level $(0.6 \mathrm{~m})$ increased by as much as $2{ }^{\circ} \mathrm{C}$ in the fogged house compared to the unfogged house. When the fans were turned off and only the fogging nozzles used, air and leaf temperatures in the fogged house dropped by as much as $4{ }^{\circ} \mathrm{C}$ (i.e., $2^{\circ} \mathrm{C}$ cooler than the unfogged house). The procedure was repeated several times over several days and each time the trend was repeated.

One possibility was that the results were caused by the mixing fans disturbing an established thermal stratification, where hotter, drier air from the upper levels of the greenhouse was being mixed with cooler, wetter air coming off the evaporative pad. Velocity measurements taken in previous years (Chapter C2), however, suggested that there were no significant vertical velocity gradients in the medium density canopy, even with evaporative pad cooling, so this possibility could not be confirmed. Measurement of air temperature as a function of height would have clarified the picture; however, sufficient instrumentation to do that did not exist and rearrangement of the existing instrumentation was not practical at that time. It was therefore decided to abandon the fogging tests and make provisions for including a follow-up test at the end of the summer to further pursue this.

\section{Methods and Materials}

In October of 1999, after summer testing was over and the plants had been removed, a twoday test was initiated to examine the vertical temperature gradients imposed when the fogging system was running. The details of the procedure are described in Chapter $\mathrm{C} 1$ and will only be briefly summarized here. On the first day, the test house was exposed to three treatments in succession: 1) fans plus fog, 2) fans without fog and 3) fog without fans. The control house used only conventional equipment. On the second day, a fourth treatment was applied to both houses where both fog and fans were off for the entire day. Evaporative pad cooling and the low ventilation rate $\left(0.041 \mathrm{~m}^{3} \mathrm{~m}^{-2} \mathrm{~s}^{-1)}\right.$ were used in both houses for both days of the test.

No plants were in either house, but the bags in which they had been grown were left in place. The aspirated stations were rearranged so as to measure vertical temperature and humidity gradients at mid-house. Two stations were located at $0.76 \mathrm{~m}$, two at $1.5 \mathrm{~m}$ and two at $2.24 \mathrm{~m}$, measured from the floor. The fogging nozzles and fans were mounted in the same location as during the earlier fogging tests, $2.1 \mathrm{~m}$ off the floor.

\section{Results}

Figure C3.1 presents the temperature differences between houses as a function of treatment. The 'base-line' treatment is from the second day when no fog or fans were used in either house. All differences are test house minus control house averaged over height and treatment such that negative values represent a cooler test house while positive values represent a warmer test house. The 'base-line' treatment shows very little temperature difference between houses at all three heights, suggesting that the houses were sufficiently similar to make comparisons between them valid.

When only fog was used the temperatures in the test house declined, more at the upper level and only slightly at the lower level, which was approximately the height of the young canopy 
during the earlier fogging test. When the fans were turned on, however, temperatures at the lower level rose by several degrees. Although the temperatures at the mid-height were relatively unaffected, those at the upper level were reduced by as much as $4{ }^{\circ} \mathrm{C}$.

Temperatures in the control house were $1.7{ }^{\circ} \mathrm{C}$ higher at the middle level and $5{ }^{\circ} \mathrm{C}$ higher at the highest level, compared to those at the lowest level, undoubtedly because of the influence of the evaporative pad. On the other hand, temperatures in the test house were approximately $1{ }^{\circ} \mathrm{C}$ less at the highest level than those at the lowest level whenever the mixing fans were running, with or without fog.

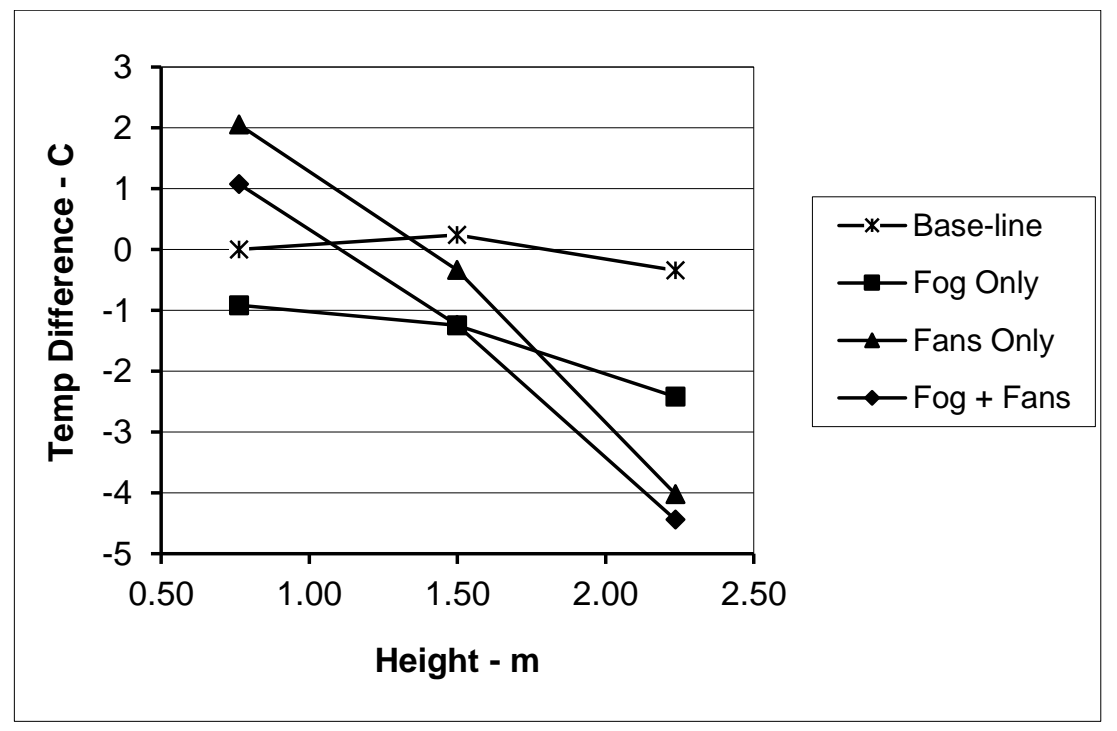

Figure C3.2: Differences in temperature between test and control houses as a function of height in the greenhouses and the treatments applied. Positive numbers indicate a warmer test house while negative numbers indicate a cooler test house.

Figure C3.2 presents dew point temperature differences between the test and control houses. There were small base-line differences approximately in line with the limits of error for the relative humidity sensors used $( \pm 2 \%)$. When the fans were on, with or without fog, dew points at the lowest level were higher in the test house than in the control house, more so with fogging than without. At the highest level, dew point temperatures were higher in the test house when both fog and fans were running but not when just the fans were running. When fog alone was used, dew points at the lowest level were only marginally increased while those at the highest level were increased a little more. Since no fogging took place, and there was no transpiration from the canopy, the increase in humidity at the upper level could only have come from the higher humidity air coming off the evaporative pad. Dew point temperatures in the control house were generally the same at the lowest and middle levels (within $0.1{ }^{\circ} \mathrm{C}$ ) but were typically 0.6 to $0.7{ }^{\circ} \mathrm{C}$ higher at the highest level that at either of the other two positions. 


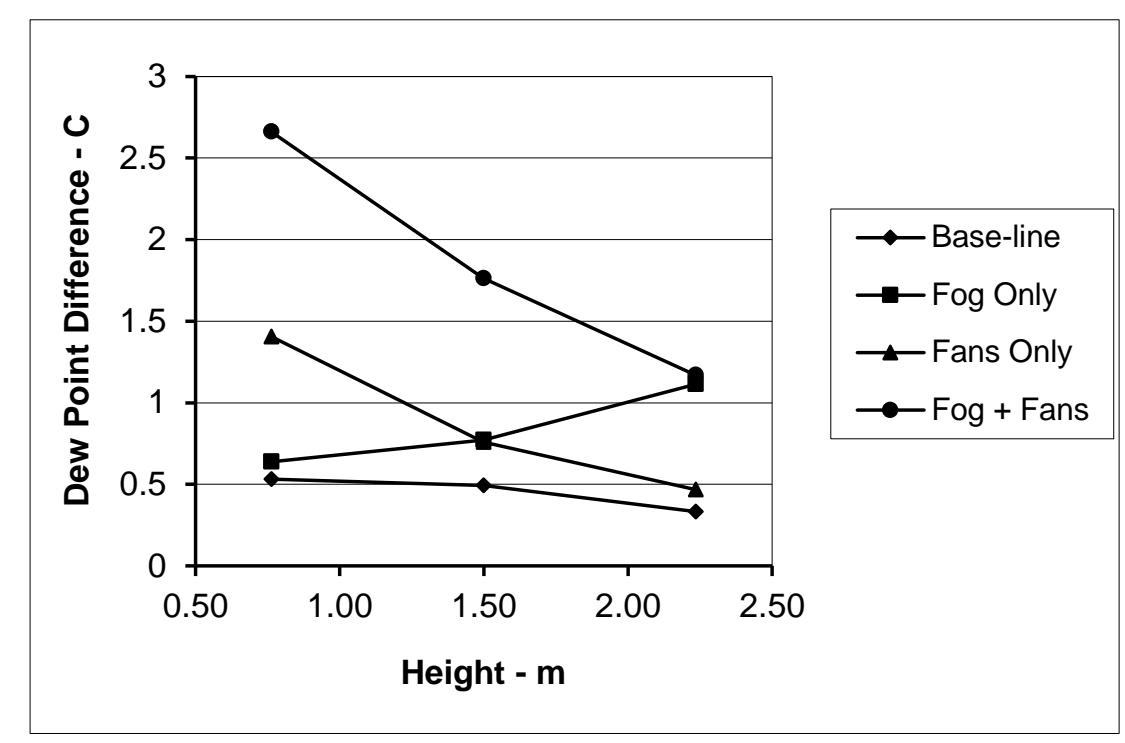

Figure C3.3: Relative humidity differences between test and control houses as a function of height in the greenhouses and the treatments applied. Positive numbers indicate a wetter test house while negative numbers indicate a drier test house.

The results clearly suggest that the evaporative pad thermally stratified the air in these tests; however, that does not conclusively prove that thermal stratification played a role in the earlier test. There were differences between the test, primarily transpiration from the canopy, but the plants were quite small and the root systems relatively undeveloped such that the effect should have been very small. A strong argument for the role of thermal stratification in the earlier test comes from the fact that air temperatures in the two tests were affected in a nearly identical way by the operation of the mixing fans; i.e., air temperatures rose at about the $0.7 \mathrm{~m}$ level whenever the mixing fans operated.

The data do suggest that fogging can be used reduce temperatures at the lower levels if it can be introduced in such a way as to not disturb stratification; however, the fogging system used here did not perform well without the fans because the droplets were too large to remain suspended on their own. An alternative fogging system would have to be used to prevent the considerable floor wetting experienced here when fans were not operating.

It might be possible to gainfully employ fans, with or without fog, if the canopy were taller. The evidence in Chapter $\mathrm{C} 2$, for example, suggests that the thermal stratification developed by evaporative pad cooling could be detrimental to the cooling of a tall crop. In that case, the upper portion of the canopy was in the hotter, drier air at the top of the house while the lower portions of the canopy were in the cooler, more humid air coming off the pad. The result was that leaf temperatures in the upper portions of the tall canopy, where most of the growth was taking place, were not much lower than in the shorter, less dense canopy. The evidence from this test suggests that mixing fans might have reversed that stratification, lowering temperatures at the top of the canopy significantly, even without fog. In fact, these tests suggest only a marginal advantage to adding fog if the mixing fans were already running.

\section{Conclusions}

Evaporative pad cooling appears to thermally stratify the air in a greenhouse to a greater 
extent than expected, based on previous velocity measurements, forcing cooler, more humid air toward the floor while leaving hotter, drier air above. The addition of mixing fans and fog apparently disturbed that stratification to a sufficient degree that cooling at the lower levels was degraded. There is some evidence, presented both in this chapter and in Chapter C2, that this behavior can be exploited to advantage in the cooling of much taller crops, but verification of that is left for further study. 


\author{
Dan H Willits \\ Biological and Agricultural Engineering, North Carolina State University
}

\title{
Abstract
}

A central hypothesis of the original proposal was that sparsely spaced plants transpire more per unit leaf area than densely spaced plants. Analysis of the NCSU transpiration data from 1997 and 1998 (Chapter A2) supported that hypothesis, but a more detailed examination was needed to see if a reason for the difference could be identified. An analysis was conducted using data only from the 1997/98 tests where the lower ventilation rate was combined with evaporative pad cooling using neither shading nor mixing fans. Transpiration per unit leaf area was again found to be higher in the lower density canopy than in the higher density canopy; however, the effect of canopy density on transpiration per plant did not follow the same trend. Transpiration per plant was higher in the high-density canopy than in the medium-density canopy but it was highest in the low-density canopy. Additional analysis suggests that the difference is attributable to intra-canopy shading. The results suggest that once a canopy closed the relationship between transpiration per plant and leaf area remained constant; however, for the low-density canopy (which never closed), transpiration per plant was much higher.

\section{Introduction}

The effects of canopy density, ventilation rate and evaporative pad cooling on transpiration were analyzed and presented in Chapter A2. Predictions from a Penman-Monteith equation were compared to the transpiration per unit leaf area from 1997 and 1998. The fit was reasonably good at high LAI, but transpiration was significantly underpredicted at low LAI. Sensitivity analysis suggested that radiation exchange was inadequately modeled by the Penman-Monteith equation; however, the evidence for this was indirect and inconclusive.

In order to better understand the relationship between transpiration and canopy density, the dataset was trimmed to test canopy effects exclusive of other treatments. The only treatments that were common across all tests during 1997/98 were low ventilation rate, evaporative pad cooling, no shade and no mixing fans. All other treatments were excluded from consideration. In addition to transpiration per unit leaf area, transpiration per unit floor area and transpiration per plant were also considered. Least-square means of the three measures of transpiration were generated from the model

\section{$\mathrm{VAR}=\mathrm{SOL}$ VPDA DEN}

using the GLM procedure in SAS (SAS, 1989), where VAR a general dependent variable representing one of the three measures of transpiration, SOL is solar energy on a horizontal surface at the top of each canopy, VPDA is the mean vapor pressure deficit of the air inside the greenhouse and DEN is a class variable representing canopy density. Mean air temperature was also considered as an independent variable, but it was not significant in most cases and was therefore not retained in the general model. The data were averaged over each half-hour, the data sampling period used in 1997, and only data from periods when solar radiation on an outside horizontal surface was greater than $500 \mathrm{~W} \mathrm{~m}^{-2}$ were considered. 


\section{Results}

Table C4.1 summarizes the least-square means generated from EQ 1 for the three measures of transpiration discussed above, along with the mean value of LAI of each canopy over the year. All of the least-square means presented were different from each other at the 0.05 level. In both years, $\mathrm{T}_{1}$ was higher in the lower density canopy than in the higher density canopy, which agrees both with the original hypothesis and the results presented in Chapter A2. The increase in $T_{1}$ in the medium-density canopy from 1997 to 1998 is the result of a $15 \%$ increase in solar energy levels in 1998 over that in 1997 plus an earlier start, and thus younger, smaller plants, during 1998. Transpiration per unit floor area, $\mathrm{T}_{\mathrm{f}}$, was higher in the higher density canopies than in the lower density canopies, which is to be expected since the higher density canopies always had a larger amount of leaf area present in the house.

The effect of canopy density on $T_{p}$ does not appear consistent. It was significantly higher in the high-density canopy than in the medium-density canopy, but it was highest in the lowdensity canopy. The fact that $T_{p}$ was higher in the low-density canopy than either of the other two is consistent with a higher $\mathrm{T}_{1}$ in the low-density canopy; however, a larger $\mathrm{T}_{\mathrm{p}}$ in the highdensity canopy compared to the medium-density canopy appears to be at odds with a lower $\mathrm{T}_{1}$ in the high-density canopy. This contradiction suggests that LAI cannot be the only factor affecting $\mathrm{T}_{\mathrm{p}}$ and that some other canopy characteristic must be involved.

Table C4.3: Least-square means of transpiration as a function of canopy density and LAI produced from tests in 1997 and 1998 where the low ventilation rate was combined with evaporative pad cooling, no shade and no mixing fans.

\begin{tabular}{llllll}
\hline Density & LAI & Year & $\begin{array}{l}\mathrm{T}_{1} \\
\mathrm{~g} \mathrm{~s}^{-1} \mathrm{~m}^{-2} \text { leaf }\end{array}$ & $\begin{array}{l}\mathrm{T}_{\mathrm{f}} \\
\mathrm{g} \mathrm{s}^{-1} \mathrm{~m}^{-2} \text { floor }\end{array}$ & $\begin{array}{l}\mathrm{T}_{\mathrm{p}} \\
\mathrm{g} \mathrm{s}^{-1} \text { plant }^{-1}\end{array}$ \\
\hline High & 2.61 & 1997 & 0.0497 & 0.0807 & 0.0309 \\
Med & 0.98 & 1997 & 0.0548 & 0.0531 & 0.0217 \\
Med & 0.94 & 1998 & 0.0908 & 0.0584 & 0.0223 \\
Low & 0.134 & 1998 & 0.1047 & 0.0162 & 0.0333 \\
\hline
\end{tabular}

Figures C4.1-C4.3 present daily averages of $T_{p}$ as a function of $A_{p}$, a representation of average plant size. Figure C4.1 presents the 1997 data where the number of plants per canopy was the same but the plants in the high-density canopy were older and larger. Apart from a rapid rise in $T_{p}$ in the medium-density canopy when the plants were very small, the rate of rise in $T_{p}$ for the two canopy densities is approximately the same. This suggests that at some point in the growth of the canopy the relationship between $T_{p}$ and $A_{p}$ approached a constant value.

Figure C4.2 presents the 1998 data where the plants in the two canopies were approximately the same size but the spacing between plants was much larger in the low-density canopy than in the medium-density canopy. Differences in $\mathrm{T}_{\mathrm{p}}$ are much less at the beginning of the dataset than at the end, but even at the beginning the low-density plants transpired more than the medium-density plants. At $A_{p}$ values less than $0.25 \mathrm{~m}^{2}$ plant $^{-1}$, for example, the least-square means of $T_{p}$ were $0.022 \mathrm{~g} \mathrm{~s}^{-1}$ plant $^{-1}$ and $0.019 \mathrm{~g} \mathrm{~s}^{-1}$ plant $^{-1}$ for the low- and medium-density canopies, respectively. It is interesting to note that the slope of $T_{p}$ in the two canopies is approximately the same until $A_{p}$ reaches about $0.30 \mathrm{~m}^{2}$ plant ${ }^{-1}$, at which point the two curves begin to diverge. 


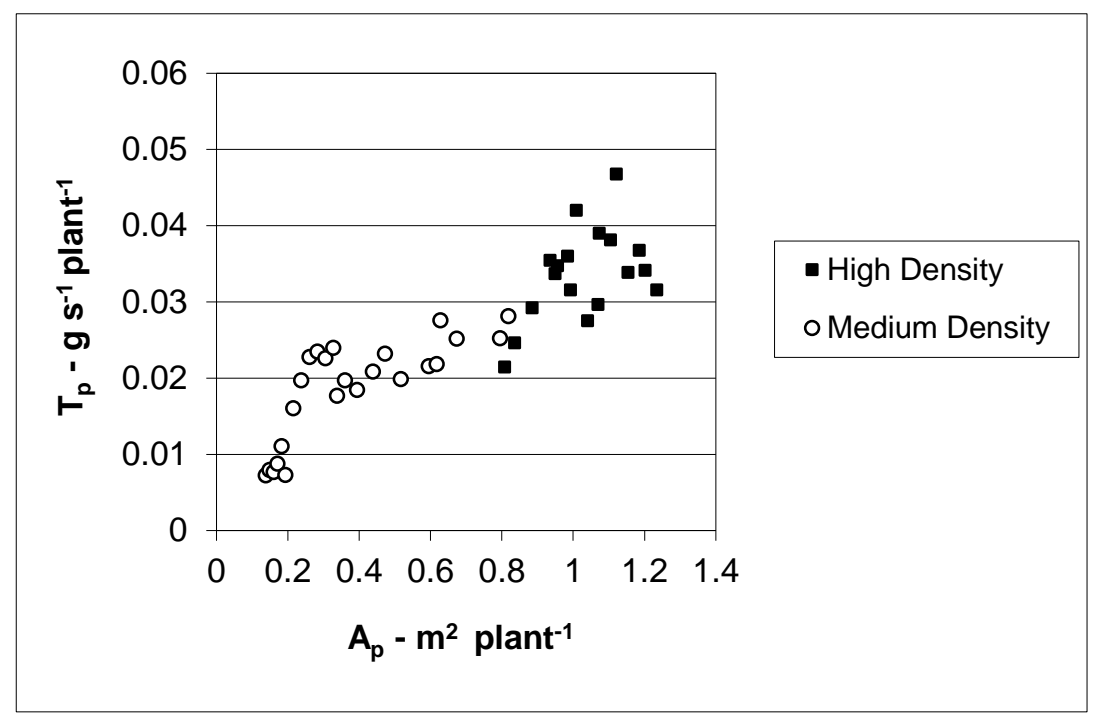

Figure C4.4: Mean daily transpiration per plant vs. leaf area per plant for 1997.

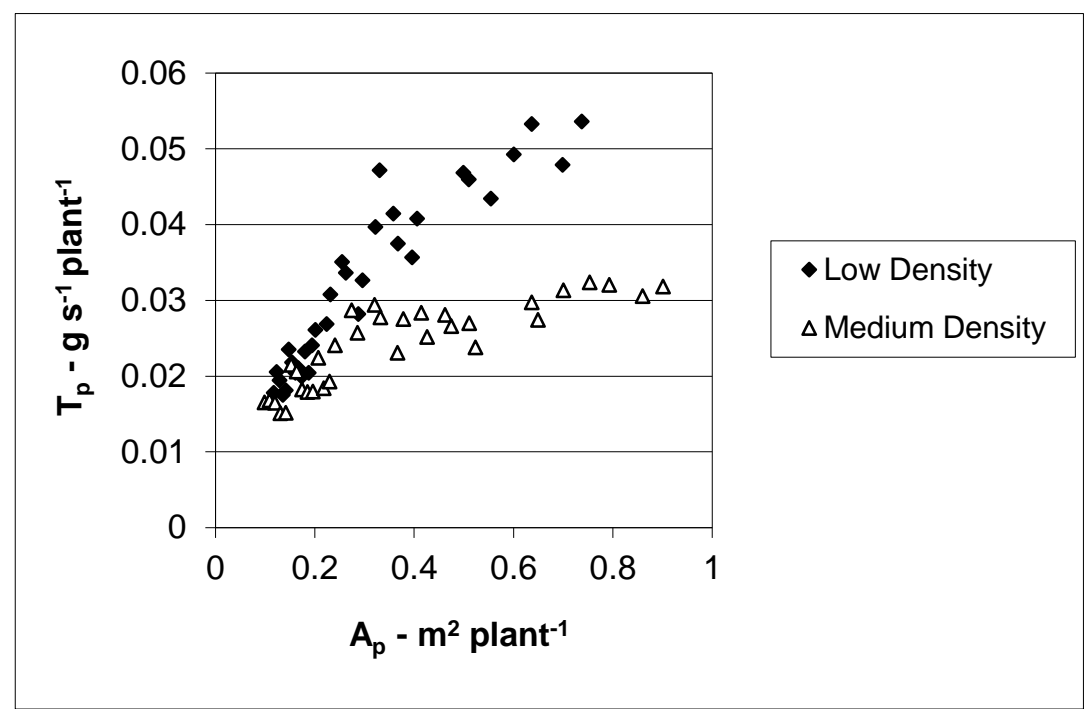

Figure C4.5: Mean daily transpiration per plant vs. leaf area per plant for 1998.

Once $A_{p}$ reached $0.30 \mathrm{~m}^{2}$ plant $^{-1}$, transpiration per plant increased much more slowly with plant size in the medium-density canopy than it did in the low-density canopy. The most likely explanation for this is the radiation exposure differences between the canopies. The plants in the low-density canopy were exposed to a fairly uniform level of long- and shortwave radiation throughout their life because any shading of the leaves on a plant came from leaves on the same plant, not from other plants. In contrast, at about the time when $A_{p}$ reached $0.30 \mathrm{~m}^{2}$ plant $^{-1}$ in the medium-density canopy, the intra-canopy and floor shading almost certainly reduced the radiant load per unit leaf area compared to that in the low-density canopy. If true, this would have also reduced $\mathrm{T}_{\mathrm{p}}$.

Figure C4.3 presents the data from both years plotted on the same graph. The slopes for the two medium-density canopies are nearly identical, and appear to be similar to the slope for the high-density canopy. Again, the slope for the low-density canopy is greater than for either of the other two densities. The agreement in slope between the two medium-density canopies and between the medium-density and high-density canopies suggests that a point was reached 
very early in canopy development after which the relationship between $T_{p}$ and $A_{p}$ remained essentially constant. It seems likely that this was due either to intra-canopy and/or floor shading or that transpiration efficiency of a certain percentage of leaves declined as new leaves were added.

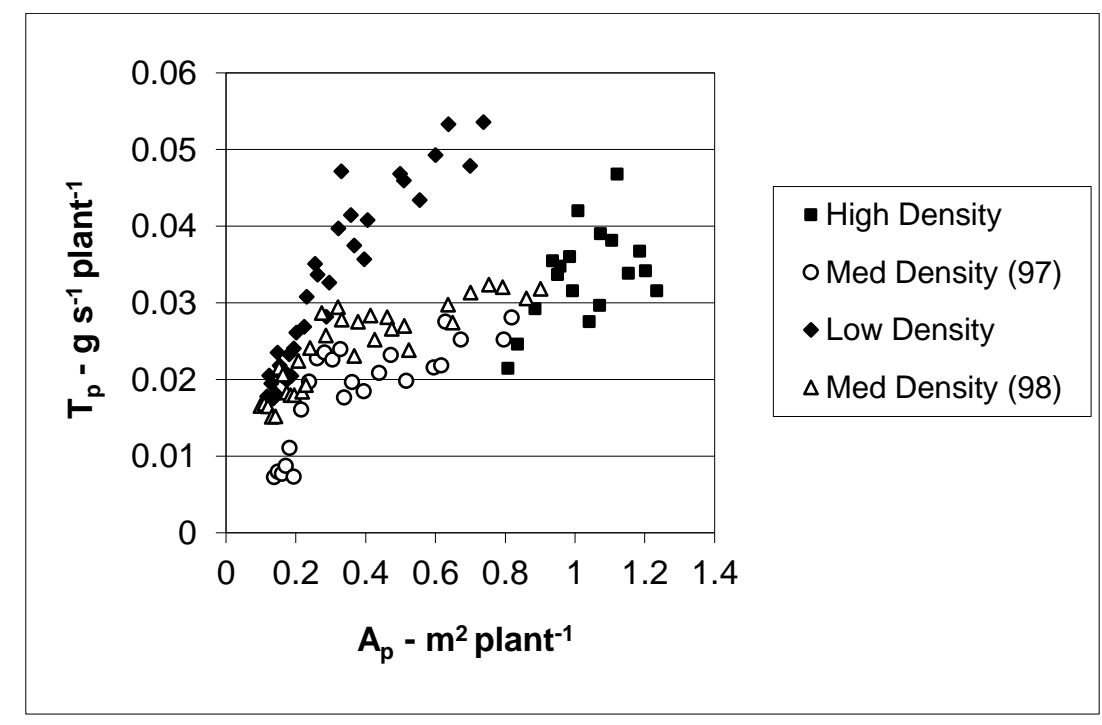

Figure C4.3: Mean daily transpiration per plant vs. leaf area per plant for both years.

The data suggest a similar relationship existed in the low-density canopy as well, but to a much lesser extent. The slope of $T_{p}$ for the low-density canopy is higher in the beginning than at the end, and it appears to approach a constant after a certain point. Intra-canopy and/or floor shading were probably not factors for plants spaced so far apart, therefore the apparent reduction in slope may have been due to a decline in transpiration efficiency of the older leaves. The length of the data record is inadequate for drawing a conclusive judgement; however, the data in Figure C4.3 strongly suggest that after some point in the development of the canopy only a percentage of the leaf area of each plant contributed to transpiration of that plant, and that the percentage was nearly constant from that point forward.

\section{Conclusions}

The results presented here suggest that LAI alone is insufficient to explain the effects of canopy density on transpiration and that correct modeling of radiation loading must also be considered. The results also suggest that at a very early stage the rate at which transpiration per plant increases with growth reaches a constant, but that rate is much lower in canopies where intra-canopy shading is possible than where it is not. These results offer some explanation as to why the Penman-Monteith equation considered in Chapter A2 did well at large values of LAI, but poorly at low values of LAI. It also suggests that a better model of radiation exchange in sparse canopies will needed if transpiration is to be accurately predicted in canopies consisting primarily of young plants.

\section{Nomenclature}


Symbol Description
A
Area, $\mathrm{m}^{2}$,
DEN
canopy density class variable,
LAI
leaf area index based on total greenhouse area, $\mathrm{m}^{2} \mathrm{~m}^{-2}$,
SOL
$\mathrm{T}$ outside solar radiation on a horizontal surface, $\mathrm{W} \mathrm{m}^{-2}$,
VAR transpiration, $\mathrm{g} \mathrm{s}^{-1}$,
VPDA a general independent variable used in EQ 1, vapor pressure deficit of the air $(\mathrm{kPa})$.

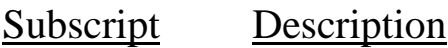

$\begin{array}{ll}\mathrm{f} & \text { per unit floor area, } \\ 1 & \text { per unit leaf area } \\ \mathrm{p} & \text { per plant. }\end{array}$

\section{References}

SAS Institute, Inc. 1989. SAS/STAT User's Guide, Version 6, Fourth Edition, Volume 2, Cary, NC: SAS Institute Inc., 846 pp. 
Ido Seginer, Moshe Tarnopolsky

Agricultural Engineering Department, Technion

\section{Abstract}

Technion's experiments were conducted in a $50 \mathrm{~m}^{2}$ greenhouse, within which were positioned two small transparent experimental chambers. Potted plants were placed in these chambers and treated by changing their arrangement, withholding irrigation, modifying the properties of the floor, misting and ventilating at different rates. The environment, as well as the canopy temperature and transpiration rate, were monitored at frequent intervals. The experiments were short-termed: treatments were often switched every day and fresh plants were brought in about once a week.

\section{Introduction}

The setup and general procedure in many of the Technion experiments was similar and, in order to minimize repetition, will be described in a separate (this) chapter. Modifications and schedules specific to the various experiments will be described, as necessary, in the following chapters.

\section{General arrangement}

The experiments were conducted in a $50 \mathrm{~m}^{2}$ greenhouse at the Technion campus in Haifa, Israel. Its $15 \mathrm{~cm}$ concrete floor was dry, and the structure was covered with a $150 \mu \mathrm{m}$ ordinary polyethylene sheet (no IR or AF additives). Greenhouse (forced) ventilation was normally kept constant at its maximum rate (about $0.05 \mathrm{~m}^{3}[$ air $] /\left(\mathrm{m}^{2}\right.$ [ground] $)$ ).

Two simple transparent chambers were constructed and positioned in the greenhouse along an east-west line, as shown in Figure T1.1. This design was meant to provide similar light integrals to the two chambers, but turned out not to be successful, because the patterns of the shadows cast by the greenhouse and neighboring structures were different at the two chamber locations and varied considerably with the season. The difference in light between the two chambers was cancelled out in most cases by switching treatments between the chambers. 


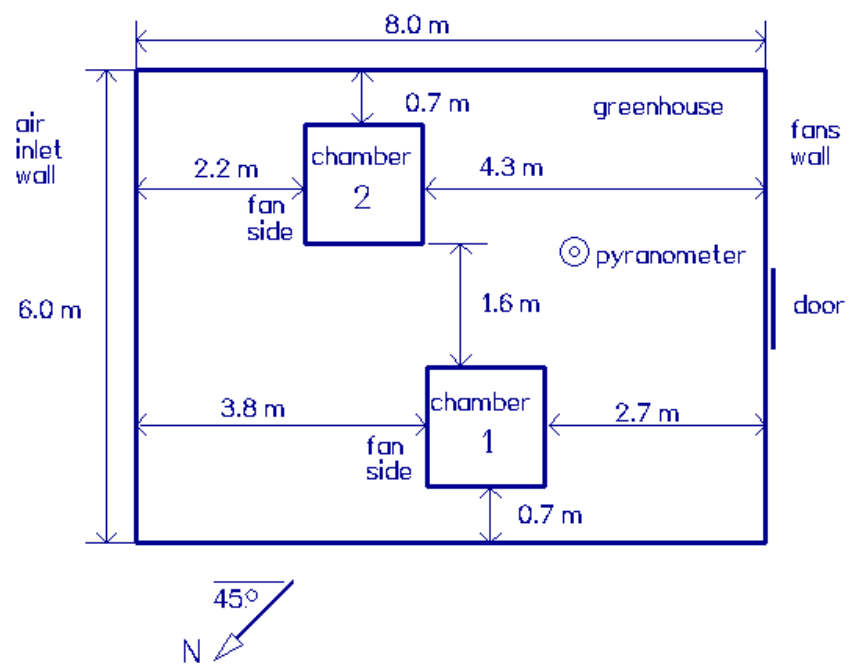

Figure T1.1: Floor plan of the greenhouse, with location of chambers.

\section{Chambers}

The chambers were $1.5 \mathrm{~m}$ on the side and $1.2 \mathrm{~m}$ high (floor area $2.25 \mathrm{~m}^{2}$, volume $2.70 \mathrm{~m}^{3}$ ), covered with the same polyethylene sheet as the greenhouse (Figure T1.2). Being protected by the greenhouse from wind forces, the construction of the chambers was very light, resulting in high light transmissivity. The overall transmissivity (greenhouse plus chamber) was $50-60 \%$, depending on chamber and season.

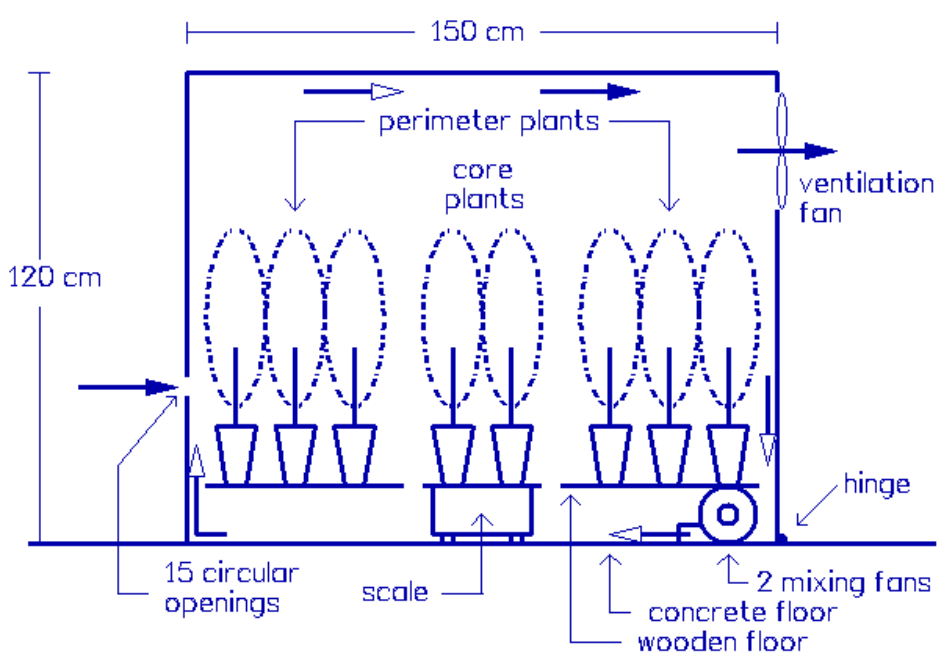

Figure T1.2: A schematic vertical cross-section of a chamber, with arrangement of plants and fans. Filled and empty arrows indicate, respectively, ventilation and mixing flow.

A wooden floor was constructed in each chamber at a height of $15 \mathrm{~cm}$ above the concrete floor. In the center of the wooden floor an opening was made to accommodate an electronic scale.

The chambers could be opened by tilting the framework (plus cover and attached instruments) around a hinge along the bottom of the north-east wall. The contact between framework and floor was sealed, as much as possible, with foam rubber. 


\section{Ventilation and mixing}

Each of the two chambers was equipped with an axial ventilation fan (EBM model AE 300$4 \mathrm{~W}, 30 \mathrm{~cm}$ diameter, nominal discharge $0.54 \mathrm{~m}^{3}[$ air] $/ \mathrm{s}$ ), mounted on the north-east wall, at a height of $100 \mathrm{~cm}$ from the concrete floor (Figure T1.2). The air was drawn in by the fan through fifteen horizontally aligned, $50 \mathrm{~mm}$ diameter openings (holes) in the opposite wall. These holes were centered at $38 \mathrm{~cm}$ above the greenhouse floor. Some air entered the chamber through the circumference of the chamber, where the walls made imperfect contact with the concrete floor.

Two centrifugal fans (model G2E 120, $10 \mathrm{~cm}$ diameter, nominal discharge $0.075 \mathrm{~m}^{3}$ [air]/s) were mounted under the wooden floor of each chamber, near the wall with the ventilation fan (Figure T1.2). These fans were used to mix the air in the chamber. Air was drawn in by the fans through a gap between the wooden floor and the ventilation-fan wall. The emerging jets moved under the floor to the other end of the chamber and went up into the chamber through a gap at the opposite (south-west) wall.

The supply of current to the ventilation and mixing fans could be regulated by changing the electrical tension between $50 \mathrm{~V}$ and $220 \mathrm{~V}$. The ventilation and mixing fans could be regulated separately. Ventilation and mixing discharges were calculated from velocity measurements with a Kurtz Mini-Anemometer Model 491. Velocity profiles were measured at three locations (Figure T1.2): (1) Horizontal component at the outlet of the ventilation fan, (2) horizontal component at the inlet holes, and (3) vertical component of the emerging mixing jet (measured from outside through the inlet holes). The difference between the discharge at (1) and (2) is an estimate of the circumferential leak at the contact with the floor.

The chambers were operated at five distinct ventilation and mixing regimes: (1) high ventilation rate, at about $0.14 \mathrm{~m}^{3} / \mathrm{s}$ through the fan, (2) low ventilation rate, at about $0.04 \mathrm{~m}^{3} / \mathrm{s}$, (3) mixing, at about $0.15 \mathrm{~m}^{3} / \mathrm{s}$ through the circulating fans, (4) no ventilation and no mixing, and (5) combined high ventilation and mixing. The last 3 ventilation regimes were only used in experiments intended to study the effect of mixing. The corresponding ventilation rates per unit floor area were about $0.06 \mathrm{~m}^{3} /\left(\mathrm{m}^{2} \mathrm{~s}\right)$ for the high ventilation rate, and about $0.02 \mathrm{~m}^{3} /\left(\mathrm{m}^{2} \mathrm{~s}\right)$ for the low rate. During the first year, experiments were conducted at the high ventilation rate, but in an attempt to enhance the temperature differences, most of the second- and third-year experiments were conducted at the low ventilation rate. Note, however, that the discharge at the low ventilation rate is only about a quarter of the design value for commercial greenhouses and the corresponding mean flow velocity, about $0.02 \mathrm{~m} / \mathrm{s}$, is an order of magnitude lower than a characteristic commercial noon-time ventilation velocity.

\section{Misting}

For the misting experiment a spraying system was installed in each of the two experimental chambers. Four 20 lit/h spray nozzles were mounted in an upward position, at the corners of a $40 \mathrm{~cm} \times 80 \mathrm{~cm}$ rectangle, $95 \mathrm{~cm}$ above the floor. At an operating pressure of $2 \mathrm{~atm}$, the characteristic drop diameter was $50 \mu \mathrm{m}$. The flow was computer-controlled via on-off solenoid valves.

\section{Plant configuration}

Throughout the project, potted plants were used. The pots were prepared at Newe Ya'ar and brought to Technion several days before the start of an experimental sequence, when the plants were 20 to $40 \mathrm{~cm}$ tall. The 0.5 liter pots were placed in holes made in $5 \mathrm{~cm}$ thick expanded polystyrene slabs, which served as lids to rectangular trays with water. The pots 
were immersed about $1-3 \mathrm{~cm}$ in the water. Normally, trays were topped up with water each morning. The surface of the substrate mixture (50\% peat and $50 \%$ vermiculite) of each pot was covered with an aluminum foil, to minimize solar heating and evaporation.

Pepper plants (cv. Mazurka) were used for the first-year experiments and tomato plants (cv. Trust) were used during the final two years. The pepper plants were grown one per pot, but in an attempt to increase the leaf area index, the number of tomato plants was increased to three per pot. In some experiments, single tomato plants per pot were also used.

Transpiration rate was usually measured by placing a tray with three or four pots on a scale. Most experiments were conducted with plant configurations designated as 'sparse' and 'dense'. The sparse configuration consisted of one tray with plants on a scale in the center of a chamber. These plants were referred to as 'core plants'. The wooden floor surrounding the core plants was usually untreated and dry. The dense configuration consisted of the same arrangement of core plants surrounded by 36 other pots with similarly prepared and watered plants. The surrounding plants were referred to as 'perimeter plants' (Figure T1.2).

\section{Instrumentation and measurements}

Measurements included outdoor weather variables (solar radiation, air temperature, and relative humidity), chamber environmental variables (solar radiation, air temperature and relative humidity), and plant variables (canopy temperature, transpiration rate and leaf area).

Weather data were collected with an automatic meteorological station, located about $30 \mathrm{~m}$ away from the greenhouse. Three pyranometers (Kipp \& Zonnen CM2) were positioned inside the greenhouse, two at the height of the top leaves near the center of each of the two chambers, and one outside the chambers, $50 \mathrm{~cm}$ above the floor, at the location indicated in Figure T1.1. Aspirated thin thermocouples and a Rotronic MP101A capacitance sensors were used to measure air temperature and humidity, respectively, in each of the chambers, as well as air temperature in the greenhouse.

Five to eight fully expanded, yet unshaded leaves, were selected for canopy temperature monitoring. Thin thermocouples (copper constantan, AWG 40 or 36, nominal diameter 0.08 or $0.13 \mathrm{~mm}, 7 \mathrm{~mm}$ twisted and soldered) were glued with a minimum amount of contact cement, over a length of $25 \mathrm{~mm}$, to the underside of the selected leaves.

Leaf area of the core plants was measured non-destructively, based on a calibration, relating the width of leaves to their area. Leaf area was usually evaluated once at the beginning and once at the end of each experimental sequence (4-6 days). At the end of a drought sequence, when the plants were wilted, they were watered, and following the quick recovery, leaf widths could be measured with confidence. Leaf area on intermediate days was estimated on the assumption that relative growth rate (RGR) of leaf area was constant during the experimental period (not always true).

The scales used to weigh the core tray-pots-plants assemblies, had a nominal capacity of about $4 \mathrm{~kg}$ and a nominal accuracy of $0.2 \mathrm{~g}$. It was assumed that the loss of water from these assemblies was equal to the transpiration rate.

The environmental data and the scales were usually logged once every 10 seconds, and 10 min means were saved on disk. In the misting experiments data were logged every $1 \mathrm{~s}$, and 30 s means were saved. 
Much of the analysis was ultimately based on averages over the four noon-time hours (10:00 to 14:00, Israel Standard Time). 
Ido Seginer, Moshe Tarnopolsky

Agricultural Engineering Department, Technion

\section{Abstract}

Previous work indicated that different plant species may have different canopy resistance and temperature under the same greenhouse conditions. In this study several experimental tests have been carried out to compare between pepper, tomato and an a-biotic evaporimeter. It was found that the plant-to-air Bowen ratio differs considerably between plant species, but that it is rather insensitive to differences in configuration, solar load and ventilation rate. A comparison with a simple greenhouse model showed that vapor continuity was a valid assumption. The results do not agree in all respects with predictions of the original (Seginer, 1994) model. Explanations for the discrepancy are offered.

\section{Introduction}

Our previous work, as well as that of others (e.g., Langton et al., 1999), suggest that different plant species, in our case pepper and tomato, respond differently to the same environment. In Chapter A6 (Seginer and Tarnopolsky, 1999), Figure 3, which has been based on the 19961998 data, shows that tomato plants are cooler than the ambient air, while pepper plants are warmer. To explore further the difference between evaporators, several experiments were conducted in 1999, involving tomato, pepper and a-biotic evaporimeters.

\section{Evaporimeters}

Evaporators differ from one another in terms of geometry, spectral properties and vapor conductance. Separating the effects of these attributes would be easiest if an artificial evaporator could be created with exactly the same geometry as the plant. Since, however, an experiment with a dense arrangement of evaporimeters was considered (requiring a large evaporating surface), a simple design had to be selected. The following is a description of one evaporimeter unit, as used in this study (Figure T2.1):

Floral porous blocks ("Supreme") were cut into $105 \mathrm{~mm} \times 75 \mathrm{~mm} \times 15 \mathrm{~mm}$ slices. Fifteen such slices, separated by filter paper sheets, were assembled into a unit. The porous material was intended to supply the evaporating surface, namely the filter paper, with water, taken up by capillary forces from a shallow reservoir. The filter paper sheets (Whatman N1), originally $270 \mathrm{~mm} \times 135 \mathrm{~mm}$ in size, were folded over cardboard squares ("Duplex", $600 \mathrm{~g} / \mathrm{m}^{2}$ ), $135 \mathrm{~mm} \times 135 \mathrm{~mm}$ in size, to increase their stiffness. Following assembly, with the filter paper protruding around the porous slices (Figure T2.1), the sequence of surfaces was: porousmaterial / filter / cardboard / filter / porous material / etc. The assembly was placed in a tray with $\sim 20 \mathrm{~mm}$ of water. Seven fine thermocouples (Chapter T1) were attached to 7 of the filter papers, and one was imbedded in the center of the unit.

The sparse evaporimeter configuration consisted of one unit in a water-filled tray on the scale. For the dense configuration, 25 more units in trays were used to form a perimeter. Other arrangements were also tried. Evaporimeters were always placed so that the direction of flow was parallel to the evaporating sheets.

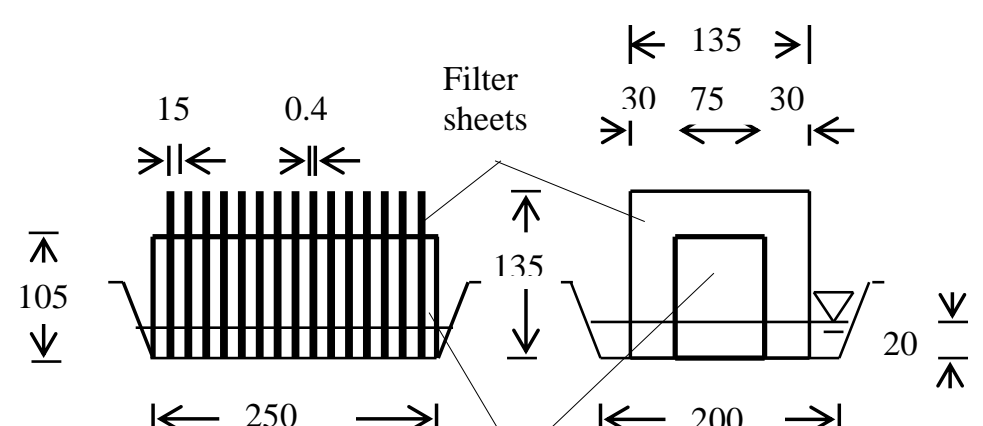


Figure T2.1: Schematic description of one evaporimeter unit.

\section{Model}

A greenhouse (chamber) model may be useful in drawing conclusions from the experimental data of this chapter. A very simple energy-balance model of a greenhouse is:

$$
e S_{o}=\rho \lambda Q\left(r_{i}-r_{o}\right)+\rho c Q\left(T_{i}-T_{o}\right)+\rho c h_{c}\left(T_{i}-T_{o}\right)
$$

where $S$ is solar radiation flux, $\mathrm{r}$ is humidity (mixing) ratio, $\mathrm{T}$ is temperature, $\rho$ is air density, $\lambda$ is latent heat of vaporization of water, $c$ is specific heat of air, $Q$ is ventilation rate, $h_{c}$ is heat conductance (radiative and convective) through the cover and e is solar heating efficiency. The subscripts $\mathrm{i}$ and $\mathrm{o}$ stand for inside and outside the greenhouse. A particular case of Equation [T2.1] is a dry greenhouse (sparse configuration taken to the limit), where

$$
\begin{aligned}
& \mathrm{eS}_{\mathrm{o}}=\rho \mathrm{c}\left(\mathrm{Q}+\mathrm{h}_{\mathrm{c}}\right)\left(\mathrm{T}_{\mathrm{i}}^{\mathrm{S}}-\mathrm{T}_{\mathrm{o}}\right), \\
& \mathrm{r}_{\mathrm{i}}^{\mathrm{S}}=\mathrm{r}_{\mathrm{o}},
\end{aligned}
$$

and the superscript $\mathrm{S}$ denotes the sparse configuration.

By adding evaporators (e.g., changing from sparse to dense configuration), the equilibrium humidity ratio, namely $\mathrm{r}_{i}$ of Equation [T2.1], increases and the air temperature decreases. Denoting the two extreme configurations by $\mathrm{S}$ and $\mathrm{D}$, and subtracting the equation for $\mathrm{D}$ from that of S, the result may be written, in an analogous form to the Bowen (sensible to latent heat) ratio, as

$$
\sigma=\frac{c\left(T_{i}^{S}-T_{i}^{D}\right)}{\lambda\left(r_{i}^{S}-r_{i}^{D}\right)}=-\frac{Q}{Q+h_{c}} .
$$

This shows that the non-dimensional slope, $\sigma$, is bounded by the range $-1 \leq \sigma \leq 0$. In terms of a psychrometric chart, this is the range of slopes between a constant enthalpy line and a constant temperature line.

For a greenhouse with a closed canopy (dense configuration) one may write the vapor continuity equation as

$$
\lambda E=\rho \lambda Q\left(r_{i}^{D}-r_{o}\right)=\rho \lambda h_{v}\left(\hat{r}_{c}^{D}-r_{i}^{D}\right),
$$


where $\mathrm{E}$ is the transpiration rate, $\hat{\mathrm{r}}_{\mathrm{c}}$ is the saturation humidity ratio at canopy temperature and $\mathrm{h}_{\mathrm{v}}$ is the vapor conductance from stomata to the greenhouse air (stomata and aerodynamic conductances). Equation [T2.5] may be rewritten as

$$
\frac{\hat{r}_{c}^{D}-r_{i}^{D}}{r_{i}^{D}-r_{o}}=\frac{Q}{h_{v}} \text {. }
$$

Another useful relation is the canopy-to-air Bowen ratio, $\beta$ :

$$
\beta=\frac{c\left(T_{c}-T_{i}\right)}{\lambda\left(\hat{r}_{c}-r_{i}\right)}
$$

The experimental data to be presented below seem to indicate that the value of $\beta$ is characteristic of the crop and is insensitive to energy load, spacing and ventilation rate. For greenhouse design purposes, knowledge of $\beta$ is presumably equivalent to knowledge of the evaporation coefficient (fraction of radiation load taken up by evaporation in the greenhouse), as used in the ASAE Standards (e.g., 1996).

Suppose that the greenhouse designer has good estimates of $\mathrm{e}, \mathrm{Q}, \mathrm{h}_{\mathrm{c}}, \mathrm{h}_{\mathrm{v}}$ and $\beta$, as well as of the critical weather, $S_{0}, T_{0}$ and $r_{0}$. Then Equations [T2.2]-[T2.4], [T2.6] and two Equations [T2.7], one for the sparse (empty) and one for the dense (closed canopy) configuration, are sufficient to calculate the six unknown quantities $T_{i}^{S}, T_{i}^{D}, T_{c}^{S}, T_{c}^{D}, r_{i}^{S}$ and $r_{i}^{D}\left(\hat{r}_{c}\right.$ is a known function of $T_{c}$ ). It is to be expected that $h_{v}$ and $\beta$ are closely related: as the stomata resistance decreases, $h_{v}$ is expected to increase and $\beta$ is expected to become more negative.

\section{Procedure}

The experiments with evaporimeters are listed in chronological order in Table T2.1. All, except the last one, were conducted with the low ventilation rate. The first data-set compares sparse and dense arrangements of evaporimeters. Data-sets 2 and 3 compare tomato plants with evaporimeters. Data-set 4 compares a complete evaporimeter unit with a single horizontal and a single vertical sheet. The last data-set compares pepper and tomato plants with evaporimeters, under both high and low ventilation rates.

The setup and general procedure are described in Chapter T1. Data-set 1 is based on experiments conducted in the standard manner, simultaneously using two chambers and switching treatments between them. All the other experiments were conducted in a single chamber, with three scales positioned side-by-side in a row perpendicular to the direction of ventilation .

Table T2.1: List of experiments which involved filter paper evaporimeters.

\begin{tabular}{llllllllll}
\hline $\begin{array}{c}\text { Data-set } \\
\text { Files }\end{array}$ & $\begin{array}{l}\text { Evapo } \\
\text {-rators }\end{array}$ & $\begin{array}{l}\text { Cham } \\
\text {-bers }\end{array}$ & $\begin{array}{l}\text { Confi- } \\
\text { guration }\end{array}$ & Nent & $\begin{array}{l}\text { Treatments } \\
\times \text { Reps }\end{array}$ & Codes & & Notes \\
\hline 1 & 990412 & E & 1,2 & S, D & L & $2 \times 8$ & ESL & EDL & \\
990418 & & & & & & & & \\
2 & 990516 & E, T & 1 & S & L & $2 \times 6$ & ESL & & TSL
\end{tabular}




\begin{tabular}{llllllllllll}
3 & 990524 & E, T & 1 & S & L & $3 \times 6$ & ESL & & TSL & TtSL & \\
990530 & & & & & & & & & & 1 1st day \\
& 990607 & & & & & & & & & 1 st day \\
4 & 990926 & E & 1 & S & L & $3 \times 4$ & ESL & EvSL EhSL & & \\
5 & 991003 & E, T, P & 1 & S & L, H & $6 \times 2$ & ESL & ESH & TSL & TSH & PSL PSH \\
\hline
\end{tabular}

Notes:

File name is date of first day of test

Evaporators: $\mathrm{P}$ - pepper, T - tomato, $\mathrm{Tt}$ - top half of tomato canopy, E - evaporimeter,

$$
\text { Ev - vertical sheet, Eh - horizontal sheet }
$$

Configuration: S - sparse, D - dense

Ventilation: $\mathrm{L}$ - low, $\mathrm{H}$ - high

1st day: Only first day of test (water supply was cut-off at end of 1st day).

\section{Results}

Figure T2.2, plotted on a psychrometric chart, compares the results for pepper and tomato of previous years (Figure 3 of Chapter A6) with the mean results of Data-set 1 for the evaporimeters. The six broken lines correspond to the six combinations of configuration (S, D) and evaporator (P, T, E). Each one of the broken lines starts on the saturation curve, with a point that describes the air in the stomata of the canopy (c). The first segment connects to the chamber air (i), the next connects to the greenhouse air (g) and finally to the outside air (o). The slopes of segments on the psychrometric chart represent the corresponding Bowen ratios.

Although the three experiments were not simultaneous, and despite the difference in weather conditions, certain features stand out rather clearly (a better impression can be gained by an imaginary parallel-shifting of the patterns, until all (i) points coincide):

1 The evaporimeters are considerably cooler (relative to chamber-air) than the plants.

2 The canopy-to-greenhouse-air segments (c-to-i) for each evaporator (plant or evaporimeter) are nearly parallel, namely independent of configuration.

3 The (finely dotted) segments connecting the chamber air (i) for the sparse and dense configurations within each pair, are nearly parallel.

4 While the pepper and tomato sparse canopy is warmer than the dense canopy, the opposite is true for the evaporimeters.

The difference between the three evaporators (Item 1 above) may be the result of both geometrical and wetness differences. The vertically and stream-wise oriented evaporimeter sheets result in a lower solar radiation load and a higher aerodynamic conductance than the more prostrate and mingled plant leaves. The evaporimeters are also obviously much wetter than the leaves (no stomata resistance). As a result of these two factors, geometry and wetness, the evaporimeters cooled considerably more than the plants.

The observation in Item 3 can be explained by Equation [T2.4], which shows that the slope $\sigma$ depends on the properties of the chamber, not on the evaporators. The mean value of $\sigma$ in Figure T2.2, based on data for the low ventilation rate, is $\sigma \cong-0.22$. Hence, the ratio of the loss of sensible heat through the cover, to the loss by ventilation, is $h_{c} / Q \cong 3.5$. The high ventilation rate is about 3 times higher than the low ventilation rate (Chapter T1), which means that for the high ventilation rate $h_{c} / Q \cong 1.2$ and $\sigma \cong-0.45$. Figure 3 of Chapter A6 
seems to support this rough estimate of $\sigma$ (note that the slope is defined by $\Delta \mathrm{T} / \Delta \mathrm{r}$, not $\Delta \mathrm{r} / \Delta \mathrm{T}$, in accordance with the definition of the Bowen ratio, $\mathrm{H} / \lambda \mathrm{E})$.

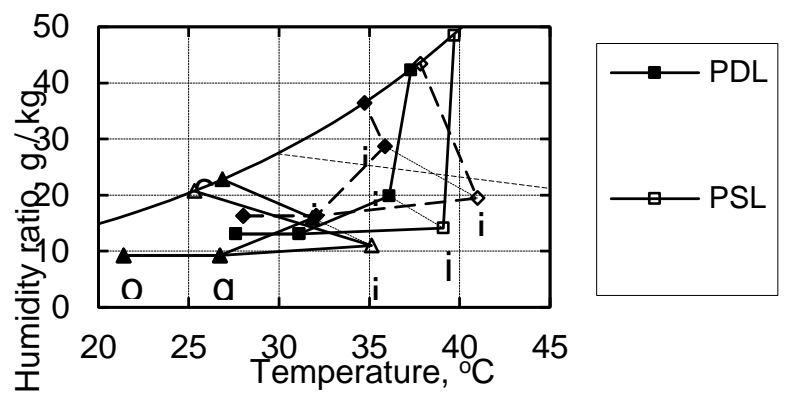

Figure T2.2: Comparison of sparse and dense configurations of pepper (1997), tomato (1998) and evaporimeters (1999). The results are plotted on a psychrometric chart. P, T and E denote Pepper, Tomato and Evaporimeter; D and S denote Dense and Sparse; c, i, g and o denote plant canopy, chamber air, greenhouse air and outside air. The top curve is the saturation function, and $100 \mathrm{~kJ} / \mathrm{kg}$ denotes a sample enthalpy line. All results are for the low ventilation rate (L).

Concerning Item 2, the shift from sparse to dense configuration does not seem to affect the canopy-to-air (c-to-i) Bowen ratio, $\beta$, as defined by Equation [T2.7]. As a result, the c-to-i enthalpy-difference decreases when changing from sparse to dense configuration. This is the consequence of either a smaller radiation load (per core plant or leaf) when the canopy is closed and (or) of a larger conductance (reduced resistance). The solar radiation load on the core plants in the dense configuration is lower than in the sparse configuration, as a result of shading by the perimeter plants. The long-wave radiation load is also smaller, because the perimeter plants are cooler than the bare floor and other radiating elements in the perimeterless (sparse) chamber. It is unlikely that the aerodynamic conductance is larger in the dense configuration, but the lower transpiration rate may (or may not) reduce the water stress and increase the stomata conductance, which may increase the total stomata-to-air conductance. The latter argument is not relevant to the evaporimeters. The reason for the higher enthalpy difference associated with the sparse evaporimeter configuration is, therefore, mostly the result of a higher radiation load.

Changing from sparse to dense configuration resulted in a reduced canopy temperature for both pepper and tomato. The evaporimeter temperature, however, increased as the density increased (Item 4). This seems to be the result of the different values of $\beta$ of the three evaporators. In the present setup, $\beta$ of tomato and $\beta$ of pepper are larger than $\sigma$, while $\beta$ of the evaporimeter is smaller. Geometrical considerations (Figure T2.2), can be used to show that this leads to the inversion of the sparse-to-dense temperature difference.

The data of Figure T2.2 were not collected simultaneously for all three evaporators. A direct comparison of the data requires pattern shifting, which it is not obvious how to do properly. To obtain a simultaneous comparison of pepper, tomato and evaporimeter, a special experiment was conducted in 1999. Two levels of ventilation, high and low, were employed in 
conjunction with the sparse configuration. The resulting Data-set 5 was used to plot Figure T2.3.

The results are very clear: the cooling effect of the evaporimeter is by far the largest, the tomato is clearly next and the pepper has the warmest canopy temperature. The effect of increasing the ventilation rate is to shift the data towards lower temperatures, here by about $4 \mathrm{~K}$. The effects of species and ventilation are similar to those obtained previously (Chapter A6, Figure 3, and Figure T2.2). The Bowen ratios between the canopies and the chamber-air are always negative, namely canopies cooler than the air. The evaporimeter was as much as $8 \mathrm{~K}$ cooler than the air, while the pepper was barely $1 \mathrm{~K}$ cooler.

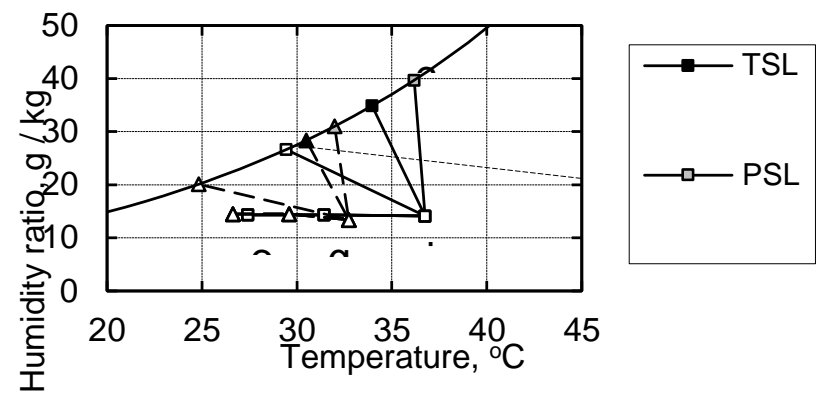

Figure T2.3: The response of pepper, tomato and evaporimeter to two ventilation regimes. $\mathrm{L}$ and $\mathrm{H}$ denote Low and High ventilation rates. Other notation as in Figure T2.2.

Data-set 4 was obtained from comparison of a standard evaporimeter unit with a single horizontal and a single vertical sheet of filter paper, all supplied with water via the porous material. Figure T2.4 shows that the geometry of the evaporator has a major effect on the results. The horizontal sheet had a considerably $(6 \mathrm{~K})$ higher temperature than the vertical sheet, probably as a result of the higher solar radiation flux, but perhaps also due to a different local ventilation pattern. The single vertical sheet was cooler by almost $2 \mathrm{~K}$ relative to the assembly of such sheets (standard unit), possibly due to a higher local ventilation rate.

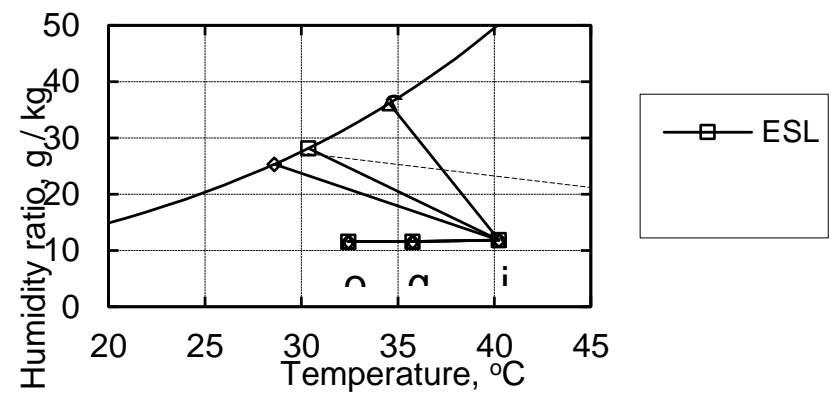


Figure T2.4: The effect of evaporimeter geometry on its temperature. Eh and Ev denote single horizontal and vertical sheets. Other notation as in Figure T2.2.

Figure T2.5 shows the results of Data-set 3, where tomato plants defoliated of their lower leaves (about half the total area) were compared with normal plants and with an evaporimeter. The temperature of the top leaves was unaffected by the defoliation.

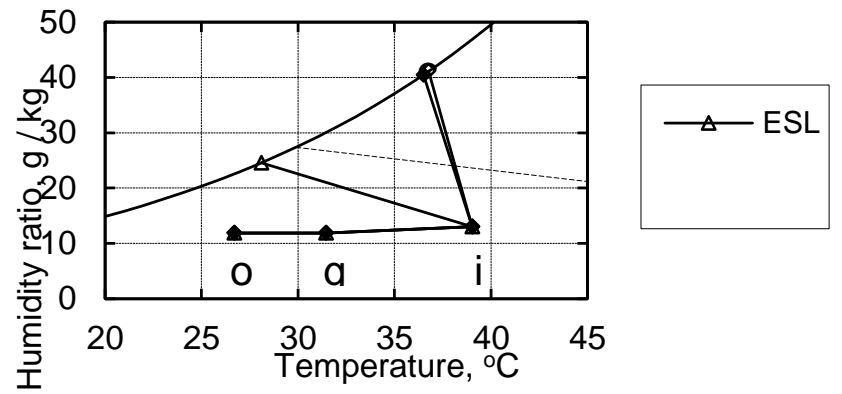

Figure T2.5: Comparison of normal and defoliated tomato plants with the evaporimeter. $\mathrm{Tt}$ denotes tomato plants with lower leaves removed. Other notation as in Figures T2.2 and T2.3.

Table T2.2 shows that the evaporator-to-air Bowen ratio differs consistently among evaporators. The overall weighted mean for pepper is 0.012 (based on 44 replications), for tomato it is -0.048 (24) and for the evaporimeters it is -0.355 (36). The effects of ventilation rate and configuration are small relative to the differences between evaporators and in view of the noise level (e.g., combination ESL in the penultimate column of the table).

Table T2.2 shows that the Bowen ratio differs consistently among evaporators. The overall weighted mean for pepper is 0.012 (based on 44 replications), for tomato it is -0.048 (24) and for the evaporimeters it is -0.355 (36). The effects of ventilation rate and configuration are small relative to the differences between evaporators and in view of the noise level (e.g., combination ESL in the penultimate column of the table).

Table T2.2: Evaporator-to-air Bowen ratios for the three evaporators - pepper, tomato and evaporimeter. Numbers in cells are the Bowen ratio and (number of replications). L, H, D and S are Low and High ventilation, Dense and Sparse configuration.

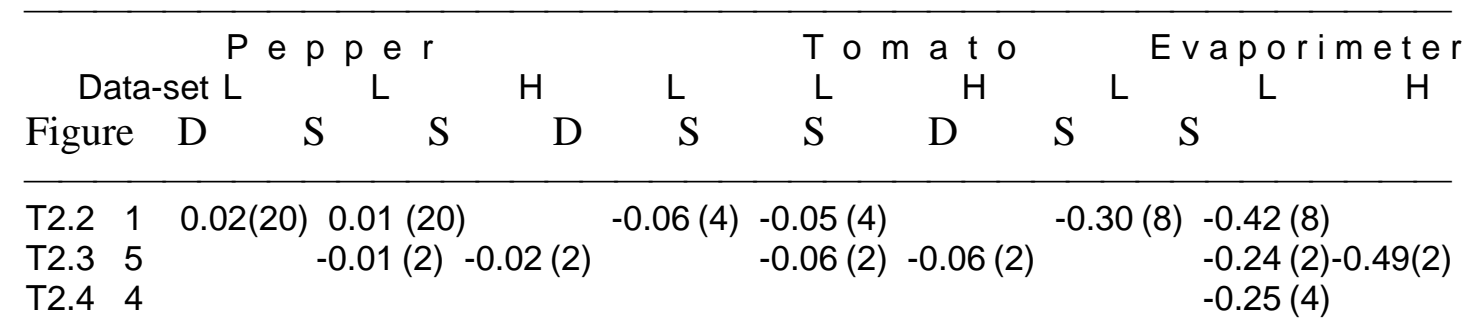




\begin{tabular}{llllllllll}
\hline mean & 0.02 & 0.01 & -0.02 & -0.06 & -0.04 & -0.06 & -0.30 & -0.37 & -0.49 \\
\hline
\end{tabular}

Notes:

Numbers in parentheses denote replications (test days). Mean is weighted by number of replications.

\section{Discussion and conclusion}

The main unexpected observation of this study is that the evaporator-to-air (c-to-i) Bowen ratio, is characteristic of the evaporator (pepper, tomato, evaporimeter; Figures T2.2, T2.3, T2.5; Table T2.2) while it is rather insensitive to configuration (Figure T2.2), ventilation rate (Figure T2.3), and solar load (Figures T2.4 and T2.5 compared to Figures T2.2 and T2.3). This observation is in contrast to the prediction of the original model (Seginer, 1994) that the evaporator-to-air segments of the sparse and dense configurations for each evaporator will meet at the saturation curve (and not be parallel).

The original model made several assumptions which are not satisfied by the experimental setup. In particular, it assumed (1) that the heat transfer through the cover is negligible and (2) that the net radiation load on the crop (per unit surface area) is independent of the configuration. Regarding Item (1), the results show that for the geometry of the chambers $\mathrm{h}_{\mathrm{c}} / \mathrm{Q} \cong 1.2 \div 3.5$, far from a negligible $\mathrm{h}_{\mathrm{c}}$. Regarding Item (2), the original model (implicitly) considered a height-less crop, with no sides to absorb radiation energy. Contrary to this assumption, our plants were tall and the load (per unit ground area) on the core plants was higher when the plants were sparse.

While the discrepancy between model predictions and experimental observation can be explained qualitatively, it is not clear, at this point, why the canopy-to-air Bowen ratio, $\beta$, is so remarkably insensitive to spacing, ventilation rate and energy load. Nevertheless, if this empirical result can be supported by independent measurements, it could be useful as a design tool, once a catalog of the characteristic Bowen ratio for different crops becomes available.

The design procedure may start by predicting the canopy temperature of an isolated plant in the following manner: Using Equations [T2.2] and [T2.3] the greenhouse air properties can be determined. Then, Equation [T2.7] may be used to find the canopy temperature, $T_{c}^{S}$. Finally, Equations [T2.4], [T2.6] and [T2.7] can be used to solve for $\mathrm{T}_{\mathrm{i}}^{\mathrm{D}}, \mathrm{r}_{\mathrm{i}}^{\mathrm{D}}$ and $\mathrm{T}_{\mathrm{c}}^{\mathrm{D}}$, which presumably describe the situation in a greenhouse with a fully developed crop.

\section{References}

ASAE. 1996. ASAE Standards, 43rd Edition. 864 pp. ASAE, St. Joseph, MI, USA.

Langton FA, Horridge JS, Hamer PJC. 1999. Effects of the glasshouse environment on leaf temperature of pot chrysanthemum and dieffenbachia. ISHS International Conference on Greenhouse Techniques towards the Third Millenium. To be published in Acta Horticulturae.

Seginer I. 1994. Transpirational cooling of a greenhouse crop with partial ground cover. Agricultural and Forest Meteorology 71:265-281.

Seginer I, Tarnopolsky M. 1999. Ground treatments to relieve heat stress of a sparse greenhouse canopy. ASAE Paper 99-4124, 16pp. 


\section{T3 RESPONSE OF PLANTS TO VENTILATION REGIMES}

Ido Seginer, Moshe Tarnopolsky

Agricultural Engineering Department, Technion

\section{Abstract}

Plants in small chambers were subjected to five ventilation regimes: (1) high ventilation rate, (2) low ventilation rate, (3) air mixing, (4) no ventilation and no mixing, and (5) combined high ventilation and mixing. The effect of mixing turned out to be negligible. Canopy temperature decreased as ventilation rate increased. The total reduction of canopy temperature from no ventilation to high ventilation was about $9 \mathrm{~K}$ for tomato and only about half of that for pepper. A simple model, which assumed that solar radiation is absorbed solely by the crop canopy, turned out to be useful only in explaining the vapor continuity but not the sensible heat continuity.

\section{Introduction}

Ventilation is the most common means of greenhouse cooling. It replaces high-enthalpy inside-air by low-enthalpy outside air. Generally speaking, properly scaled ventilation experiments must satisfy at least three conditions:

1 There should be a geometrical similarity between model and prototype.

2 The ventilation flux per unit incoming solar energy, namely per unit floor area, must be the same in model and prototype.

3 The transfer coefficient between canopy and bulk air, namely the local flow velocity, must also be the same in model and prototype.

Obviously, the first condition was not satisfied in this project, since the proportions of the chambers were very different from those of a commercial greenhouse. As shown in Chapter $\mathrm{T} 2$, this resulted in an unusually high rate of heat loss trough the cover of the chambers (not by ventilation). An attempt was made, nevertheless, to explore the feasibility of balancing properly the last two items, by adding a mixing fan to the chamber.

\section{Scaling}

Suppose that the length, width and height of a prototype fan-ventilated greenhouse are $\mathrm{L}_{\mathrm{p}}, \mathrm{W}_{\mathrm{p}}$ and $\mathrm{H}_{\mathrm{p}}$, respectively, and that the corresponding values for the model are $\mathrm{L}_{\mathrm{m}}, \mathrm{W}_{\mathrm{m}}$ and $\mathrm{H}_{\mathrm{m}}$. The specific ventilation rate (per unit floor area), q, is calculated from the ventilation discharge, $Q$, via

$$
\mathrm{q}=\mathrm{Q} /(\mathrm{LW})
$$

so that the condition for having the same specific ventilation rate in model and prototype is

$$
\mathrm{Q}_{\mathrm{m}} / \mathrm{Q}_{\mathrm{p}}=\mathrm{L}_{\mathrm{m}} \mathrm{W}_{\mathrm{m}} /\left(\mathrm{L}_{\mathrm{p}} \mathrm{W}_{\mathrm{p}}\right) \text {. }
$$

The flow velocity in the greenhouse is calculated via 


$$
\mathrm{v}=\mathrm{Q} /(\mathrm{WH}),
$$

so that the condition for the same velocity in model and prototype is

$$
\mathrm{Q}_{\mathrm{m}} / \mathrm{Q}_{\mathrm{p}}=\mathrm{H}_{\mathrm{m}} \mathrm{W}_{\mathrm{m}} /\left(\mathrm{H}_{\mathrm{p}} \mathrm{W}_{\mathrm{p}}\right) .
$$

Often, as in the present case, where the chambers are considered to be models of greenhouses, $\mathrm{L}_{\mathrm{m}} / \mathrm{L}_{\mathrm{p}} \ll \mathrm{H}_{\mathrm{m}} / \mathrm{H}_{\mathrm{p}}$. This means that if $\mathrm{q}$ is scaled properly, the velocity and the resulting canopy transfer coefficient in the model, are too small. To increase the transfer coefficient in the model (chamber), without affecting q, mixing of the inside air can be added as required.

\section{Modeling}

A very simple chamber-in-greenhouse model may be based on energy conservation equations of the system. The energy balance of an essentially dry greenhouse is

$$
\varepsilon_{\mathrm{g}} \mathrm{S}_{\mathrm{o}}=\rho \mathrm{c}\left(\mathrm{h}_{\mathrm{g}}+\mathrm{q}_{\mathrm{g}}\right)\left(\mathrm{T}_{\mathrm{g}}-\mathrm{T}_{\mathrm{o}}\right)
$$

where $\mathrm{S}$ is solar radiation, $\mathrm{T}$ is temperature, $\varepsilon$ is solar heating efficiency, $\rho$ is air density, $\mathrm{c}$ is specific heat of air, $h$ is heat transfer coefficient through the cover, and the subscripts $g$ and $o$ denote 'greenhouse', and 'outside'. The energy balance of a chamber with a dense plant configuration is

$$
\varepsilon_{\mathrm{i}} \mathrm{S}_{\mathrm{o}}=\rho c\left(\mathrm{~h}_{\mathrm{i}}+\mathrm{q}_{\mathrm{i}}\right)\left(\mathrm{T}_{\mathrm{i}}-\mathrm{T}_{\mathrm{g}}\right)+\rho \lambda \mathrm{q}_{\mathrm{i}}\left(\mathrm{r}_{\mathrm{i}}-\mathrm{r}_{\mathrm{g}}\right),
$$

where $\mathrm{r}$ is humidity ratio, $\hat{\mathrm{r}}$ is $\mathrm{r}$ at saturation, $\lambda$ is latent heat of vaporization of water and the subscript $i$ denotes the chamber. The energy balance of the canopy is

$$
\varepsilon_{\mathrm{i}} \mathrm{S}_{\mathrm{o}}=\rho \mathrm{ch}_{\mathrm{c}}\left(\mathrm{T}_{\mathrm{c}}-\mathrm{T}_{\mathrm{i}}\right)+\rho \lambda \mathrm{h}_{\mathrm{v}}\left(\hat{\mathrm{r}}_{\mathrm{c}}-\mathrm{r}_{\mathrm{i}}\right),
$$

where $h_{c}$ and $h_{v}$ are the canopy-to-chamber-air conductances for sensible and latent heat $\left(\mathrm{h}_{\mathrm{v}} \leq \mathrm{h}_{\mathrm{c}}\right)$ and the subscript $\mathrm{c}$ denotes 'plant canopy'. Finally, the vapor continuity from canopy to chamber-air and from there to the greenhouse air, is

$$
\rho \lambda h_{v}\left(\hat{r}_{c}-r_{i}\right)=\rho \lambda q_{i}\left(r_{i}-r_{o}\right)
$$

Assuming that the various transfer coefficients are at most functions of the chamber ventilation rate, $\mathrm{q}_{\mathrm{i}}$, simple relations between the variables are obtainable, as follows

$$
\mathrm{S}_{\mathrm{o}}=\mathrm{A}\left(\mathrm{T}_{\mathrm{g}}-\mathrm{T}_{\mathrm{o}}\right) \quad \text { where } \quad \mathrm{A} \equiv \frac{\rho \mathrm{c}\left(\mathrm{h}_{\mathrm{g}}+\mathrm{q}_{\mathrm{g}}\right)}{\varepsilon_{\mathrm{g}}}
$$

$$
\frac{\left(\hat{r}_{c}-r_{i}\right)}{\left(r_{i}-r_{g}\right)}=F \quad \text { where } \quad F\left\{q_{i}\right\} \equiv \frac{q_{i}}{h_{v}}
$$

$$
\frac{\left(\hat{\mathrm{r}}_{\mathrm{c}}-\mathrm{r}_{\mathrm{i}}\right)}{\left(\hat{\mathrm{r}}_{\mathrm{c}}-\mathrm{r}_{\mathrm{g}}\right)}=\mathrm{G} \quad \text { where } \quad \mathrm{G}\left\{\mathrm{q}_{\mathrm{i}}\right\}=\frac{\mathrm{F}}{\mathrm{F}+1} \equiv \frac{1}{1+\mathrm{h}_{\mathrm{v}} / \mathrm{q}_{\mathrm{i}}}
$$

$\mathrm{G}$ varies between 0 and 1 . When $\mathrm{h}_{\mathrm{v}} / \mathrm{q}_{\mathrm{i}} \rightarrow 0$ (high ventilation, low transpiration), $\mathrm{G} \rightarrow 1$. When $\mathrm{h}_{\mathrm{v}} / \mathrm{q}_{\mathrm{i}} \rightarrow \infty$ (low ventilation, high transpiration), $\mathrm{G} \rightarrow 0$. 
[T3.6] $\mathrm{S}_{\mathrm{o}}=\mathrm{B}\left(\mathrm{T}_{\mathrm{i}}-\mathrm{T}_{\mathrm{g}}\right)+\mathrm{C}\left(\mathrm{r}_{\mathrm{i}}-\mathrm{r}_{\mathrm{g}}\right)$ where $\mathrm{B}\left\{\mathrm{q}_{\mathrm{i}}\right\} \equiv \frac{\rho \mathrm{c}\left(\mathrm{h}_{\mathrm{i}}+\mathrm{q}_{\mathrm{i}}\right)}{\varepsilon_{\mathrm{i}}}, \mathrm{C}\left\{\mathrm{q}_{\mathrm{i}}\right\} \equiv \frac{\rho \lambda \mathrm{q}_{\mathrm{i}}}{\varepsilon_{\mathrm{i}}}$

[T3.7] $\mathrm{S}_{\mathrm{o}}=\mathrm{D}\left(\mathrm{T}_{\mathrm{c}}-\mathrm{T}_{\mathrm{i}}\right)+\mathrm{E}\left(\hat{\mathrm{r}}_{\mathrm{c}}-\mathrm{r}_{\mathrm{i}}\right) \quad$ where $\mathrm{D}\left\{\mathrm{q}_{\mathrm{i}}\right\} \equiv \frac{\rho \mathrm{ch}}{\varepsilon_{\mathrm{c}}}, \mathrm{E}\left\{\mathrm{q}_{\mathrm{i}}\right\} \equiv \frac{\rho \lambda \mathrm{h}_{\mathrm{v}}}{\varepsilon_{\mathrm{i}}}$

[T3.12],[T3.13] $\mathrm{B}\left(\mathrm{T}_{\mathrm{i}}-\mathrm{T}_{\mathrm{g}}\right)+\mathrm{C}\left(\mathrm{r}_{\mathrm{i}}-\mathrm{r}_{\mathrm{g}}\right)=\mathrm{D}\left(\mathrm{T}_{\mathrm{c}}-\mathrm{T}_{\mathrm{i}}\right)+\mathrm{E}\left(\hat{\mathrm{r}}_{\mathrm{c}}-\mathrm{r}_{\mathrm{i}}\right)$

$$
\left(\hat{r}_{\mathrm{c}}-\mathrm{r}_{\mathrm{i}}\right)=\mathrm{F}\left(\mathrm{r}_{\mathrm{i}}-\mathrm{r}_{\mathrm{g}}\right)
$$

[T3.14],[T3.15] $\mathrm{B}\left(\mathrm{T}_{\mathrm{i}}-\mathrm{T}_{\mathrm{g}}\right)+\mathrm{C}\left(\mathrm{r}_{\mathrm{i}}-\mathrm{r}_{\mathrm{g}}\right)=\mathrm{D}\left(\mathrm{T}_{\mathrm{c}}-\mathrm{T}_{\mathrm{i}}\right)+\mathrm{EF}\left(\mathrm{r}_{\mathrm{i}}-\mathrm{r}_{\mathrm{g}}\right)$

$$
\mathrm{C}=\mathrm{EF}
$$

[T3.16],[T3.17] $\frac{\left(T_{c}-T_{i}\right)}{\left(T_{i}-T_{g}\right)}=H=\frac{B}{D}=\frac{h_{i}+q_{i}}{h_{c}}$

[T3.18] $\frac{\left(\mathrm{T}_{\mathrm{c}}-\mathrm{T}_{\mathrm{i}}\right)}{\left(\mathrm{T}_{\mathrm{c}}-\mathrm{T}_{\mathrm{g}}\right)}=\mathrm{I} \quad$ where $\quad \mathrm{I}\left\{\mathrm{q}_{\mathrm{i}}\right\}=\frac{\mathrm{H}}{\mathrm{H}+1} \equiv \frac{1}{1+\mathrm{h}_{\mathrm{c}} /\left(\mathrm{h}_{\mathrm{i}}+\mathrm{q}_{\mathrm{i}}\right)}$

As will be seen later, A was found to be a function of the season, $G$ - a function of the ventilation rate (as expected), but I turned out to be intractable.

\section{Procedure}

For procedural details see Chapter T1. Comparisons between various ventilation regimes were carried out in each of the three years of the project. All comparisons utilized the dense configuration (chamber filled with plants), so that the model would approximately apply. Five regimes were utilized (1) high ventilation rate $(\mathrm{H})$, at about $0.14 \mathrm{~m}^{3} / \mathrm{s}$ through the fan (about $0.06 \mathrm{~m}^{3} /\left(\mathrm{m}^{2} \mathrm{~s}\right)$ ), (2) low ventilation rate $(\mathrm{L})$, at about $0.04 \mathrm{~m}^{3} / \mathrm{s}$ (about $0.02 \mathrm{~m}^{3} /\left(\mathrm{m}^{2} \mathrm{~s}\right)$, (3) no ventilation and no mixing $(\mathrm{N}),(4)$ mixing $(\mathrm{N}+\mathrm{M})$, at about $0.15 \mathrm{~m}^{3} / \mathrm{s}$ through the circulating fans, and (5) combined high ventilation and mixing $(\mathrm{H}+\mathrm{M})$. Altogether, 44 tests were made, mostly in pairs (both chambers used). The various treatments are summarized in Table T3.1.

Table T3.1: Number of tests per combination of crop, period and ventilation

\begin{tabular}{|c|c|c|c|c|c|}
\hline $\begin{array}{l}\text { Crop } \\
\text { Year } \\
\text { Period* }^{*}\end{array}$ & $\begin{array}{l}\text { Pepper } \\
1997 \\
23\end{array}$ & $\begin{array}{c}\text { Tomato } \\
1998 \\
2\end{array}$ & $\begin{array}{c}\text { Tomato } \\
1999 \\
3\end{array}$ & \multicolumn{2}{|c|}{$\begin{array}{l}\text { Evaporimeter } \\
1999 \\
1\end{array}$} \\
\hline $\begin{array}{l}\text { Ventilation } \\
\text { regime }\end{array}$ & & & & & Total \\
\hline High $(\mathrm{H})$ & 24 & 2 & 2 & & 10 \\
\hline Low (L) & 12 & 8 & 4 & 8 & 23 \\
\hline None $(\mathrm{N})$ & & 2 & & & 2 \\
\hline $\operatorname{Mix}(\mathrm{N}+\mathrm{M})$ & 12 & 2 & 2 & & 7 \\
\hline $\mathrm{H}+\mathrm{M}$ & & 2 & & & 2 \\
\hline Total & 12 & 16 & 8 & 8 & 44 \\
\hline
\end{tabular}
regime. The combination $\mathrm{L}+\mathrm{M}$ was not tried.

*Periods: 1, 2 and 3 - around Julian days 100, 200 and 300.

\section{Results: psychrometric charts}

It became clear, at an early stage, that the only significant differences were between the 
regimes $\mathrm{H}, \mathrm{L}$ and $\mathrm{N}$. $\mathrm{M}$ was hardly distinguishable from $\mathrm{N}$, and $\mathrm{H}+\mathrm{M}$ was hardly distinguishable from $\mathrm{H}$. In other words: the effect of mixing, despite its high discharge, was rather limited. This is illustrated in Figure T3.1, which is a plot, on a psychrometric chart (as in Chapter T2), of the 1998 Tomato results. The four pairs of tests shown in the figure (low ventilation being the common treatment), were carried out under similar, but not identical weather conditions. Note that the clustering of the data for $\mathrm{H}, \mathrm{L}$ and $\mathrm{N}$ becomes even more evident if the data represented by triangles are shifted to the right, and those with diamonds to the left (and up), to fit the outdoor conditions of the other tests. Note that the humidity ratio is always highest in the canopy and lowest in the (dry) greenhouse (same as outdoors). This results in a value of $\mathrm{G}$ which is constrained between 0 and 1, as expected, the lower values being associated with the unventilated chamber (thin lines). The order of temperatures is not as clear: $\mathrm{T}_{\mathrm{c}}-\mathrm{T}_{\mathrm{i}}$ is sometimes positive and sometimes negative.

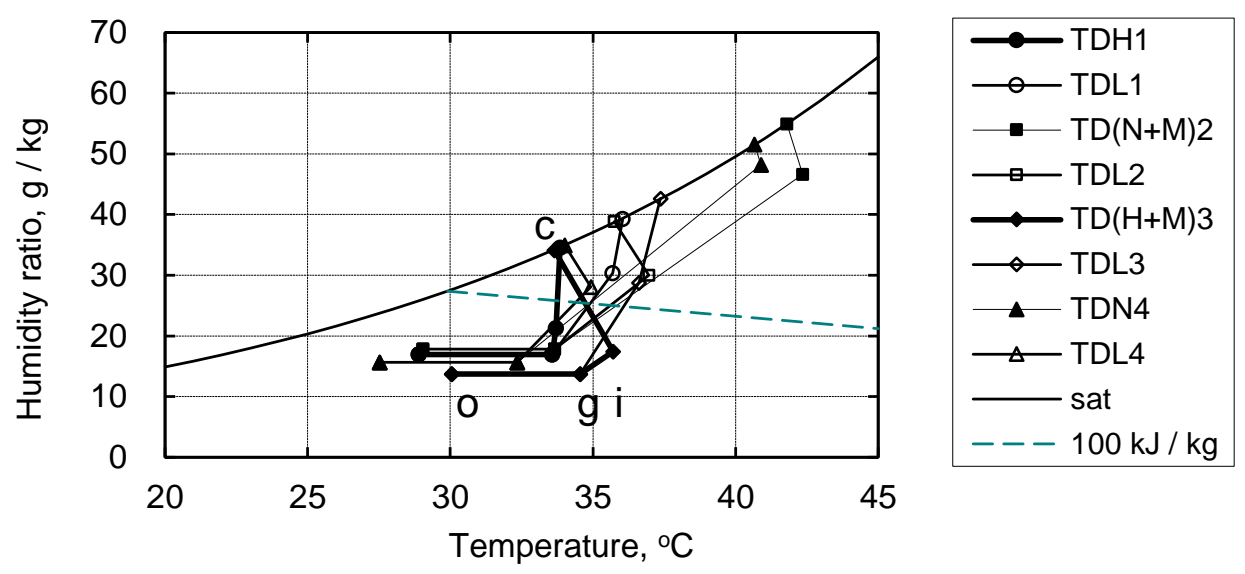

Figure T3.1: Tomato experiments, 1998. The results are plotted on a psychrometric chart. c, i, g and o denote plant canopy, chamber air, greenhouse air and outside air. The top curve is the saturation function, and $100 \mathrm{~kJ} / \mathrm{kg}$ denotes a sample enthalpy line. Heavy lines indicate high ventilation $(\mathrm{H}$, and $\mathrm{H}+\mathrm{M})$, medium lines - low ventilation $(\mathrm{L})$, and thin lines - no ventilation $(\mathrm{N}$ and $\mathrm{N}+\mathrm{M})$. Points represent 4-hour (10-14 IST) means.

Figure T3.2 shows the 1997 results for pepper. The data can be separated first into the two periods (Table T3.1), Period 2 being characterized by higher solar radiation. Higher solar radiation results in a longer segment o-to-g and pushes the canopy temperature up. The four lines of Period 2 are ordered as expected, and the canopy temperature is higher than the chamber temperature. The data of Period 3 can be further split into two pairs, one (diamonds) for low $\left(27^{\circ} \mathrm{C}\right)$ outdoors temperature and the other (triangles) for high $\left(33^{\circ} \mathrm{C}\right)$ temperature (and somewhat higher solar radiation). When shifted relative to one another, the data of Period 3 also appear to be reasonably well arranged. An important qualitative difference between the data of the two periods is that the c-to-i slope of Period 2 is positive and that of Period 3 is negative (the tomato data of Figure T3.1 have an intermediate slope). This is what one would expect to be the effect of changes in solar radiation. It is not clear, however, what is the source of heat for the chamber air (i) in Period 3. Since the chamber air is warmer than both the canopy and the greenhouse air, it must be heated from elements of the chamber that are not the canopy. Another possibility is that the measured temperature of the core plants (Chapter T1) is lower than the temperature of the perimeter plants. The situation where the temperature of the chamber air is the highest in the system, is not compatible with the simple model of the previous section. 

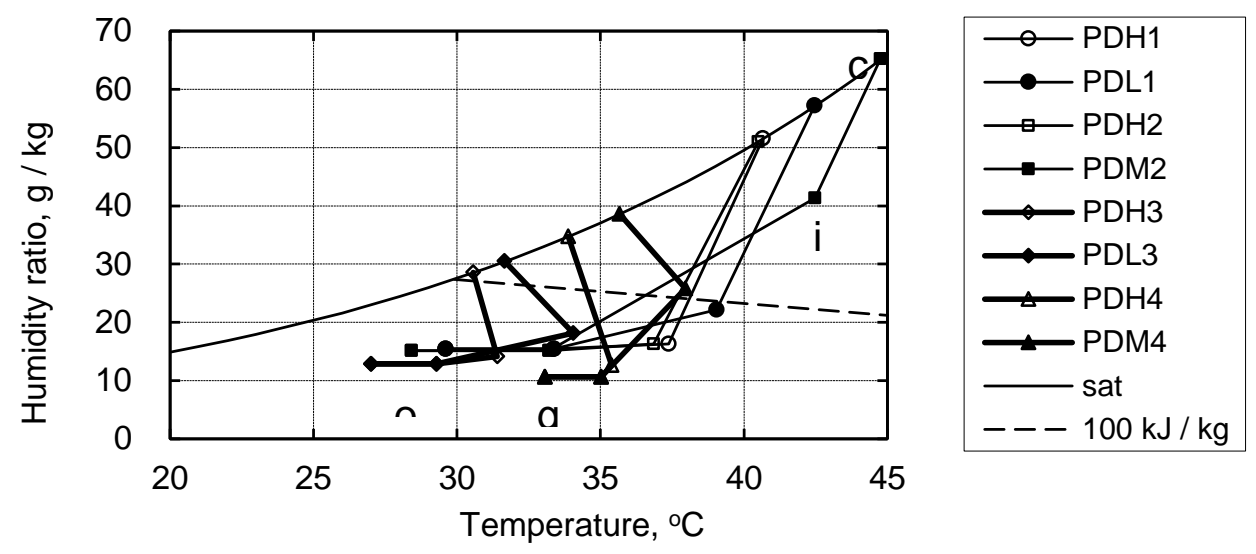

Figure T3.2: Pepper experiments, 1997. Notation is the same as in Figure T3.1. Medium lines represent results of Period 2, and heavy lines indicate Period 3.

\section{Results: model factors}

The factors A, G and I were evaluated from the 4-hour means of the various measured variables. A (Equation [T3.9]), which relates to the greenhouse, and therefore is not significantly affected by the ventilation regimes in the chambers, turned out to be strongly dependent on the season. As can be seen in Figure T3.3, the cooling factor, A, is lower in spring than in autumn. What seems to be a straight line, is probably a segment of a harmonic curve, which repeats itself over the years. If the variation was solely due to the movement of the solar trajectory, March and September would have had the same A. Since, based on the data, this is not likely, perhaps some other climatic factors are responsible for the perturbation.

The factor $\mathrm{G}$ (Equation T3.13]) compares canopy vapor conductance with the ventilation rate and is expected to depend both on the crop and on the ventilation regime. Table T3.2 summarizes the results of $\mathrm{G}$, which are expected to be between 0 (low ventilation, high transpiration) and 1. Despite the limited number of tests and the large variability, a clear pattern emerges. As expected, $\mathrm{G}$ increases with ventilation rate. There is also an indication that pepper has a lower canopy conductance than tomato, which agrees with results in Chapters A6 and T2 (note that differences for tomato between years are relatively small). The evaporimeter results (Chapter T2) are similar to the results for tomato, and not lower, as expected. There is a systematic difference between chambers, the values of $\mathrm{G}$ for chamber 2 being consistently lower.

The factor I (Equation [T3.19]) is expected to behave in a similar manner to G (Equation [T3.11]), namely to be limited to the range 0 to 1 . This, however, would only happen if either $\mathrm{T}_{\mathrm{c}}>\mathrm{T}_{\mathrm{i}}>\mathrm{T}_{\mathrm{g}}$ (positive Bowen ratio) or $\mathrm{T}_{\mathrm{g}}>\mathrm{T}_{\mathrm{i}}>\mathrm{T}_{\mathrm{c}}$ (negative Bowen ratio). In fact, in a few cases $T_{c}>T_{g}>T_{i}$ and in many cases $T_{i}$ is the highest temperature, situations that the simple model does not allow. As a result, no generalization could be drawn, at this stage, from the results for I. 


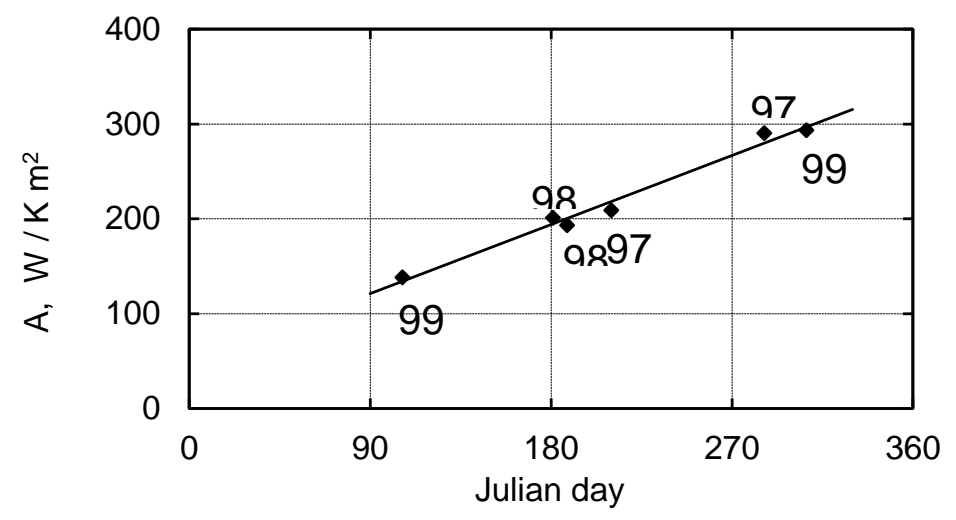

Figure T3.3: The greenhouse cooling factor, A, as a function of the season.

\section{Discussion and conclusion}

The effect of ventilation is well known: canopy temperature decreases as ventilation rate increases. In this respect, the results (Figures T3.1 and T3.2) agree with the expectations. Tomato (Figure T3.1) seems to respond better than pepper (Figure T3.2) to ventilation, the total reduction of temperature from no ventilation $(\mathrm{N})$ to high ventilation $(\mathrm{H})$ being about $9 \mathrm{~K}$ for tomato and only about half of that for pepper.

Table T3.2: Mean values of the factor $G$ for all the experiments. Numbers in parentheses are number of replications.

\begin{tabular}{|c|c|c|c|c|c|c|c|c|}
\hline \multirow{3}{*}{$\begin{array}{l}\text { Crop } \\
\text { Year } \\
\text { Chamber } \\
\text { Ventilation } \\
\text { regime }\end{array}$} & \multicolumn{2}{|c|}{$\begin{array}{l}\text { Pepper } \\
1997\end{array}$} & \multicolumn{2}{|c|}{$\begin{array}{c}\text { Tomato } \\
1998\end{array}$} & \multicolumn{2}{|c|}{$\begin{array}{c}\text { Tomato } \\
1999\end{array}$} & \multicolumn{2}{|c|}{$\begin{array}{c}\text { Evaporimeter } \\
1999\end{array}$} \\
\hline & 1 & 2 & 1 & 2 & 1 & 2 & 1 & 2 \\
\hline & & & & & & & & \\
\hline $\operatorname{High}(\mathrm{H})$ & $0.95(4)$ & $0.89(2)$ & $0.86(2)$ & $0.69(2)$ & $0.90(1)$ & $0.79(1)$ & & \\
\hline Low (L) & $0.80(2)$ & $0.68(1)$ & $0.50(4)$ & $0.31(4)$ & $0.63(1)^{*}$ & $0.43(2)$ & 0.55 & $0.47(4)$ \\
\hline $\operatorname{Mix}(\mathrm{M})$ & $0.50(2)$ & $0.39(1)$ & $0.21(2)$ & $0.10(2)$ & $0.25(1)$ & $-0.09(1)$ & & \\
\hline Ratio 2/1 & \multicolumn{2}{|c|}{0.87} & \multicolumn{2}{|c|}{0.70} & \multicolumn{2}{|c|}{0.63} & \multicolumn{2}{|c|}{$0.85^{\star *}$} \\
\hline \multicolumn{9}{|c|}{ Means of two chambers } \\
\hline $\operatorname{High}(\mathrm{H})$ & \multicolumn{2}{|c|}{0.92} & \multicolumn{2}{|c|}{0.78} & \multicolumn{2}{|c|}{0.85} & \multirow{3}{*}{\multicolumn{2}{|c|}{0.51}} \\
\hline Low (L) & \multirow{2}{*}{\multicolumn{2}{|c|}{$\begin{array}{l}0.74 \\
0.45\end{array}$}} & \multirow{2}{*}{\multicolumn{2}{|c|}{$\begin{array}{l}0.41 \\
0.16\end{array}$}} & \multirow{2}{*}{\multicolumn{2}{|c|}{$\begin{array}{l}0.53 \\
0.08\end{array}$}} & & \\
\hline $\operatorname{Mix}(\mathrm{M})$ & & & & & & & & \\
\hline \multicolumn{2}{|l|}{ Overall mean } & 0.70 & \multicolumn{2}{|c|}{0.45} & \multicolumn{2}{|c|}{0.49} & \multicolumn{2}{|c|}{$0.51^{* *}$} \\
\hline
\end{tabular}

* One suspect result ignored.

** Only L.

Adding a mixing fan did not produce a measurable difference on the few occasions that it has been tested (Figures T3.1 and T3.2). Mixing may be an important unifying agent at night or a means to destroy stable stratification, but here it did not change the transfer coefficients in a way that could be sensed by the canopy. 
The simple model, which assumed that solar radiation is absorbed solely by the crop canopy (or floor), turned out to be useful only in explaining the vapor continuity (Table T3.2) but not the sensible heat continuity. 
Ido Seginer, Moshe Tarnopolsky

Agricultural Engineering Department, Technion

\section{Abstract}

The canopy of small tomato plants was subjected to 1-min misting pulses once an hour. Comparisons were made with sparsely and densely spaced non-misted plants. Misting was an effective cooling method ( $2 \mathrm{~K}$ on average), but a dense configuration was twice as effective as misting for sparsely arranged plants. Doubling the misting frequency could increase somewhat the cooling effect. The canopy to chamber-air Bowen ratio was negative and about equal for all treatments.

\section{Introduction}

Misting, the wetting of a canopy to induce effective evaporative cooling, is often used in greenhouse production, despite the dangers of salt deposition and pathogen development. To minimize the dangers, the method if often used only as an emergency measure under high heat load conditions. In Chapters A6 and T2 it has been shown that, in summer, sparse canopies are exposed to harsher conditions than dense canopies. Misting, therefore, could help to reduce the temperature of sparse canopies.

An experiment was conducted during Summer 1999 to find how effective is misting in reducing canopy temperature under conditions of high energy load.

\section{Procedure}

The experimental facility and the general procedures have been described in Chapter T1. For the misting experiment a spraying system has been installed in each of the two chambers. Four $20 \mathrm{lit} / \mathrm{h}$ spray nozzles were mounted in an upward position, at the corners of a $40 \mathrm{~cm} \times$ $80 \mathrm{~cm}$ rectangle, $95 \mathrm{~cm}$ above each chamber's floor. At an operating pressure of $2 \mathrm{~atm}$ the characteristic drop diameter was $50 \mu \mathrm{m}$. The flow was computer controlled via solenoid valves.

Misting was applied automatically for 1 minute every 60 minutes. This was meant to provide a fully wetted canopy on the one hand and sufficient drying time on the other hand. Data were sampled once every second, and 30s means were stored for further analysis, much of which was based on 4-hour (10:00-14:00 IST) means of all variables.

Each replication of the experiment consisted of a 4-day sequence (Table T4.1), and involved the two experimental chambers, as well as a sparse and a dense plant configuration (Chapter T1). The emphasis in the design was on the misting effect. Note that the effects of chamber and coreplants group (A or B) are confounded, and that the two configurations (S and D) are not compared on the same days.

Three replications of the experimental sequence were carried out, each using a fresh batch of plants. The mean weather conditions during the noon period (10:00-14:00 IST) are shown in Table T4.2.

Table T4.1: A single experimental 4-day sequence. A and B denote two similar 
groups of core plants, D and S are Dense and Sparse plant configurations, $\mathrm{M}$ and $\mathrm{O}$ are Misting and $\mathrm{nO}$ misting.

\begin{tabular}{|c|c|c|c|c|c|c|}
\hline & \multicolumn{3}{|c|}{$\begin{array}{l}\text { Chamber } 1 \\
\text { Core plants A }\end{array}$} & \multicolumn{3}{|c|}{$\begin{array}{l}\text { Chamber } 2 \\
\text { Core plants B }\end{array}$} \\
\hline & Config & Mist & Code & Config & Mist & Code \\
\hline Day 1: & Dense & Yes & ADM & Dense & No & BDO \\
\hline Day 2: & Dense & No & ADO & Dense & Yes & BDM \\
\hline Day 3: & Sparse & Yes & ASM & Sparse & No & BSO \\
\hline Day 4: & Sparse & No & ASO & Sparse & Yes & BSM \\
\hline
\end{tabular}

Table T4.2: Experimental schedule. $\mathrm{S}_{\mathrm{o}}, \mathrm{T}_{\mathrm{ao}}$ and $\mathrm{T}_{\mathrm{do}}$ are noon-time means of outdoors solar radiation, air temperature and dew-point temperature.

\begin{tabular}{lllll}
\hline Replication & Dates & So & $\mathrm{T}_{\mathrm{ao}}$ & $\mathrm{T}_{\mathrm{do}}$ \\
\hline 1 & 27-30 Jun 99 & 914 & 27.9 & 21.1 \\
2 & 04-07 Jul 99 & 922 & 28.2 & 22.2 \\
3 & 25-28 Oct 99 & 562 & 25.4 & 14.3 \\
\hline
\end{tabular}

Obviously, the last replication was carried out at a much lower energy load.

\section{Results and discussion}

A sample canopy temperature trajectory is presented in Figure T4.1. As soon as misting was applied, leaf and air temperature dropped by about $4 \mathrm{~K}$ and the dew-point temperature rose by about $7 \mathrm{~K}$. Then these temperatures slowly returned to their pre-spray levels. The dew-point trajectory behaved smoothly, but the canopy temperature repeatedly followed a characteristic irregular pattern, and took the full hour to reach its pre-spray level. The pattern may be the consequence of measuring 8 individual leaves, with different wetting a exposure characteristic. The unexpected decline in temperature, during the second quarter hour after spraying, may reflect the dynamic response to local dripping and drying.

A summary of the responses to the various treatments is shown in Table T4.3. The transpiration rate, E, was extracted from the weight-time trajectories (e.g., bottom of Figure T4.1), on the assumption that the vertical segments represent the addition of water by misting, while the down sloping segments represent transpiration. Since the rate of water deposition ( $4000 \mathrm{~g} /(\mathrm{h} \cdot 9$ plants $)$ ) was so much larger than the rate of transpiration ( $180 \mathrm{~g} /(\mathrm{h} \cdot 9$ plants $)$, this approximation seems justified.

The table shows that the difference between chambers and plants was small (Line 1). Chamber 1 was somewhat drier than Chamber 2, leading to a somewhat higher transpiration rate. The difference between the dense and the sparse configuration (Line 2) was larger than the effect of misting (Line 5). Air and canopy temperature were about $4 \mathrm{~K}$ lower in the dense configuration, while the mean cooling effect of misting was only about $2 \mathrm{~K}$. Transpiration rate of the core plants was reduced from $\sim 200 \mathrm{~g} /(\mathrm{h} \cdot 9$ plants) to $\sim 100 \mathrm{~g} /(\mathrm{h} \cdot 9$ plants), by adding the 


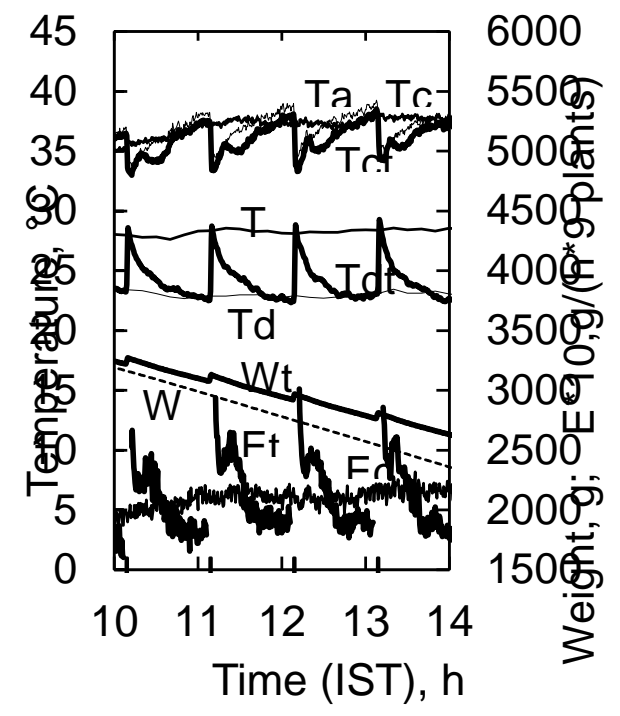

Figure T4.1: Four spray events at hourly intervals. The upper part shows mean temperatures and the lower part shows the transpiration rates. $\mathrm{T}$ is temperature, $\mathrm{W}$ is weight of pots and $\mathrm{E}$ is the transpiration rate $(-\mathrm{dW} / \mathrm{dt})$. The first subscripts of $\mathrm{T}$, namely a, $d$ and $c$ denote air, dew-point and canopy. The second subscript of $T$ and the subscripts of $\mathrm{W}$ and $\mathrm{E}$, namely $\mathrm{o}, \mathrm{t}$ and $\mathrm{c}$ denote outdoors, treatment and control.

Table T4.3: Mean differences between treatments. Noon time (10:00-14:00 IST) means over three replications. $\mathrm{T}_{\mathrm{ai}}$ is air temperature inside chambers; $\mathrm{T}_{\mathrm{di}}$ is dewpoint temperature inside chambers; $\mathrm{T}_{\mathrm{c}}$ is canopy temperature; $\mathrm{E}$ is transpiration rate.

\begin{tabular}{|c|c|c|c|c|c|c|c|}
\hline Line & Treatments & $\begin{array}{r}\Delta \mathrm{T}_{\mathrm{ai}} \\
\mathrm{K}\end{array}$ & $\begin{array}{r}\Delta \mathrm{T}_{\mathrm{di}} \\
\mathrm{K}\end{array}$ & $\begin{array}{r}\Delta \mathrm{T}_{\mathrm{c}} \\
\mathrm{K}\end{array}$ & $\begin{array}{r}\left(T_{d i}-T\right. \\
K\end{array}$ & $\begin{array}{r}\Delta\left(\mathrm{T}_{\mathrm{c}}-\right. \\
\mathrm{K}\end{array}$ & $\begin{array}{l}\Delta \mathrm{E} \\
\text { (h.9plants }\end{array}$ \\
\hline 1 & $(1+A)-(2+B)$ & 0.2 & -1.4 & 0.2 & -1.7 & 0.0 & 12 \\
\hline 2 & D-S & -4.4 & 2.3 & -3.6 & 6.7 & 0.8 & -97 \\
\hline 3 & DO-SO & -4.8 & 2.8 & -4.1 & 7.6 & 0.7 & -96 \\
\hline 4 & DM-SM & -4.0 & 1.8 & -3.0 & 5.8 & 1.0 & -98 \\
\hline 5 & M-O & -1.8 & 1.6 & -1.7 & 3.5 & 0.2 & 22 \\
\hline 6 & DM-DO & -1.4 & 1.2 & -1.1 & 2.6 & 0.3 & 21 \\
\hline 7 & SM-SO & -2.3 & 2.1 & -2.2 & 4.4 & 0.0 & 23 \\
\hline
\end{tabular}
1, 2 Chambers 1 and 2
A, B Core plants A and B
$D, S$ Dense and Sparse configuration
$\mathrm{M}, \mathrm{O}$ Mist and no-mist

perimeter plants (dense configuration). Misting, on the other hand, added about 20 $\mathrm{g} /(\mathrm{h} \cdot 9$ plants) to evapotranspiration. Partitioning of the measured evapotranspiration between 
evaporation of deposited water and transpired water was not possible in our setup.

The interaction between configuration and misting is shown by the numbers in regular (not bold) font. There seems to be a somewhat larger difference between the dense and sparse configuration when no misting was applied (Line 3) than with misting (Line 4). This is also reflected in the results of Lines 6 and 7.

The data which were used to produce Table T4.3, were also subjected to an analysis of variance (ANOVA). The model, in SAS notation (SAS Institute, 1989), was

$$
X=R C(R) P(R) T
$$

where $\mathrm{R}$ denotes the replication (weather), $\mathrm{C}$ is configuration, $\mathrm{P}$ is plants \& chamber, and $\mathrm{T}$ is the misting treatment. $\mathrm{C}$ and $\mathrm{P}$ were considered to be nested within the weather blocks. Table T4.4 summarizes this analysis.

Table T4.4: Summary of analysis of variance. * indicates that an effect is significant at the $5 \%$ level

\begin{tabular}{|c|c|c|c|c|c|c|c|c|c|c|c|c|c|c|}
\hline \multirow{2}{*}{\multicolumn{4}{|c|}{$\begin{array}{l}\text { Number of } \\
\text { of levels } \\
\text { of effects } \\
\text { R C P T }\end{array}$}} & \multirow{2}{*}{\multicolumn{2}{|c|}{$\begin{array}{l}\text { Variable } \\
\text { Units }\end{array}$}} & \multirow[t]{2}{*}{ Mean } & $\begin{array}{c}\text { Residua } \\
\text { RMS } \\
\text { error }\end{array}$ & $\begin{array}{l}\text { al } \\
\text { Relative } \\
\text { error }\end{array}$ & e $R^{2}$ & & $\begin{array}{l}\text { sign } \\
\text { of }\end{array}$ & $\begin{array}{l}\text { ifican } \\
\text { effect }\end{array}$ & $\begin{array}{l}\text { nce } \\
\text { ts }\end{array}$ & \\
\hline & & & & & & & $\%$ & & & model $\mathrm{R}$ & $R$ & $C(R)$ & $P(R)$ & \\
\hline 3 & 2 & 6 & 2 & $S_{i}$ & $\mathrm{~W} / \mathrm{m}^{2}$ & 479 & 11.5 & 2.4 & 0.997 & * & * & * & * & \\
\hline 3 & 2 & 6 & 2 & $\mathrm{~T}_{\mathrm{ai}}$ & ${ }^{\circ} \mathrm{C}$ & 32.5 & 0.5 & & 0.994 & * & * & * & - & \\
\hline 3 & 2 & 6 & 2 & $\mathrm{~T}_{\mathrm{di}}$ & ${ }^{\circ} \mathrm{C}$ & 21.7 & 0.7 & & 0.975 & * & * & * & * & \\
\hline 3 & 2 & 6 & 2 & $\mathrm{~T}_{\mathrm{c}}$ & ${ }^{\circ} \mathrm{C}$ & 31.2 & 0.5 & & 0.992 & * & * & * & - & * \\
\hline 3 & 2 & 6 & 2 & $\mathrm{E}$ & $\mathrm{g} /$ (h.9plants) & 0.271 & 0.020 & 7.3 & 0.947 & * & * & * & * & * \\
\hline
\end{tabular}

$$
\begin{array}{ll}
\mathrm{R}=\text { replication } & \mathrm{P}=\text { plant } \& \text { chamber } \\
\mathrm{C}=\text { configuration: Dense or Sparse } & \mathrm{T}=\text { treatment: Mist or NoMist }
\end{array}
$$

Table T4.4 shows a very good fit of the proposed model. Almost all effects are significant at the 5\% level and the residual errors are rather small. The only three insignificant effects are (1) effect of misting on the solar radiation model, $(2,3)$ effect of the interaction between plant $\&$ chamber and the weather, on the air and canopy temperature. The first is rather expected, since only a major change in albedo could affect the solar radiation measurement. Regarding the other two, $T_{a i}$ and $T_{c}$ are well correlated $\left(T_{a i}-T_{c}\right.$ small, Table $\left.T 4.3\right)$ and mainly affected by the ventilation rate. The significant effect of $\mathrm{P}$ on $\mathrm{S}_{\mathrm{i}}$ is due to different shadow patterns at the location of the two chambers, and the significant effects of $\mathrm{P}$ on $\mathrm{T}_{\mathrm{di}}$ and $\mathrm{E}$ are probably due to differences between the misting systems.

Figure T4.2 summarizes the results in a plot on a psychrometric chart (as in Chapters T2 and T3). Each point is the mean of 6 ( 2 chambers $\times 3$ reps) noon-time (10:00-14:00 IST) results. The four broken lines correspond to the four combinations of configuration (S, D) and treatment $(\mathrm{M}, \mathrm{O})$. 


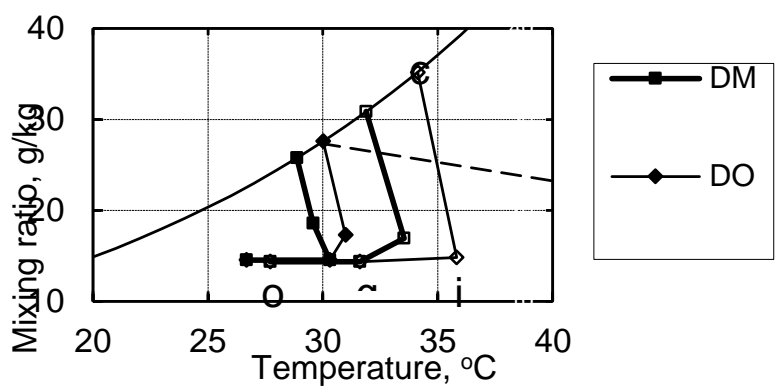

Figure T4.2: Mean performance for the four combinations of configuration and treatment, all at low ventilation rate. The results are plotted on a psychrometric chart. D and S denote Dense and Sparse; $\mathrm{M}$ and $\mathrm{O}$ denote Mist and nO-mist; c, i, g and o denote plant canopy, chamber air, greenhouse air and outside air. The top curve is the saturation function. $100 \mathrm{~kJ} / \mathrm{kg}$ is a sample constant enthalpy line.

An atmospheric-air-temperature difference of about $1 \mathrm{~K}$ is evident between the dense and the sparse configuration tests. Correcting for this discrepancy by an imaginary shifting, the picture is still very clear:

1 Inside-chamber temperatures ( $\mathrm{i}$ and $\mathrm{c}$ ) decrease from the sparse and no-mist combination, SO, through SM and DO to DM, the dense configuration with misting. The total reduction in canopy temperature (accounting for the difference in weather) is about $6 \mathrm{~K}, 4 \mathrm{~K}$ of which are the result of the difference between sparse and dense configuration. The effect of misting on the sparsely spaced plants is about $2 \mathrm{~K}$, while the effect on the dense configuration is only about $1 \mathrm{~K}$.

2 The negative slopes (negative Bowen ratio) of the stomata-to-greenhouse-air (c to i) segments are almost parallel. This repeats itself in other experiments (e.g., Chapter A6, Figure 3 ), but does not agree with our expectations. The expectation was that the sparse configuration would have a more negative slope than the dense one, because the vapor pressure deficit of the sparse crop is larger. Misting was also expected to produce a more negative slope, due to a lower canopy resistance. Obviously, the slope of these segments is much less negative than the constant-wet-bulb (or constant-enthalpy) lines, due to the solar load on the canopy.

3 The effect of dense canopy and of misting on the chamber air is similar to the effect of a wet pad: All result in a cooler, but more humid environment. In the absence of heat load, the chamber air would cool along the wet-bulb slope.

\section{Conclusions}

1 The mean cooling effect of misting was significant (about $2 \mathrm{~K}$ ) but not dramatic, despite the very dry greenhouse air (Figure T4.2).

2 Doubling the misting frequency could further reduce the mean temperature to a total difference of perhaps 3K (Figure T4.1). 
3 The canopy to chamber-air Bowen ratio was negative and about equal for all treatments (Figure T4.2). 
Ido Seginer, Moshe Tarnopolsky

Agricultural Engineering Department, Technion

\section{Abstract}

A procedure for monitoring transpiration and canopy temperature of potted tomato plants subjected to drought, is described. Four drought sequences were carried out in the summer of 1998 and five sequences in the summer of 1999. Each sequence was 4 to 7 days long, irrigation being terminated at the end of the first day. The results showed that the transpiration rate decreased by about half over each subsequent day. The noon-time canopy temperature of the stressed plants increased on the first day by about $4 \mathrm{~K}$ and reached a maximum deviation from normal of about $6 \mathrm{~K}$. Canopy temperature and transpiration were about equally sensitive to stress. No significant differences were observed between intact plants and plants whose lower half-canopy was removed.

\section{Objective}

In agricultural practice in general, and for high-value greenhouse crops in particular, early detection of stress is of primary importance. Stress may have different causes, such as water deficit, high temperature or a disease. In the context of sparse canopy in the greenhouse, the focus is on water stress, which results from evaporative demand in excess of the supply capacity of the crop-soil system. A significant water stress expresses itself in measurable changes of plant attributes, such as reduced transpiration and photosynthesis fluxes, increased temperature and sensible heat flux, reduced water content and turgor, and reduced chlorophyll fluorescence. The practical problem is to find an attribute which is easy to measure automatically and which can provide an early warning of stress. Here the focus is on automatic monitoring of transpiration rate and canopy temperature and the question is which of these two would provide an earlier reliable warning. Once this question is answered, the next step is to develop useful procedures based on this finding. One possible application is a stress warning system for commercial greenhouses. Another possible application is a procedure to distinguish between plants with different drought-coping capabilities (Chapters $\mathrm{Y} 1$ and $\mathrm{Y} 2)$.

\section{Procedure}

This report refers to the experiments of summer 1998 (August to September) and summer 1999 (April to May), which differed somewhat in terms of procedure. The setup and general procedure are described in Chapter T1. Ventilation rate was at the low level in both years.

In 1998 irrigated and stressed tomato plants were compared. The irrigated plants were placed in one of the chambers and the stressed plants in the other chamber. Four 0.5 liter pots, each with a single tomato plant, $20-30 \mathrm{~cm}$ tall, were placed in holes made in a $5 \mathrm{~cm}$ thick expanded polystyrene sheet, which served as a lid to a rectangular tray with water. The surface of the soil mixture (50\% peat and $50 \%$ vermiculite) of each pot was covered with an aluminum foil, to minimize solar heating and evaporation. Two such trays were prepared and placed one on each of the two scales (one in each chamber). Following one calibration day with a layer of water in both trays, water was drained from one of the trays. The other tray was topped up once a day. Four drought sequences were conducted, each 3 to 6 days long from drainage of 
extra water to termination.

In 1999 the treatments were compared in a single chamber, to circumvent the difficulty of different light patterns in the two chambers. Three scales were placed side-by-side in the chamber, so that the ventilation flow was perpendicular to the line of scales. One of the scales measured the evaporation from an evaporimeter unit (Chapter T2) and the other two were used for various treatments. Five drought sequences were conducted, 3 to 5 days long from initial drainage to termination. Of these, three were conducted with standard tomato plants, and two - with plants whose lower half-canopy was removed ('pruned' or 'defoliated' plants).

Evaporation and canopy temperature measurements, as well as environmental measurements, were as described in Chapters $\mathrm{T} 1$ and $\mathrm{T} 2$.

Table T5.1 lists all the relevant drought sequences. Note that the reference in 1998 is irrigated tomato plants (in the other chamber) while in 1999 it is an evaporimeter (in the same chamber as the stressed plants).

Table T5.1: List of drought sequences. $\mathrm{T}$ is Tomato, $\mathrm{N}$ is Normal plant, $\mathrm{P}$ is Pruned (lower canopy), $\mathrm{D}$ is drought treatment, $\mathrm{I}$ is irrigated and $\mathrm{E}$ is evaporimeter.

\begin{tabular}{|c|c|c|c|c|c|c|c|}
\hline \multirow{3}{*}{$\begin{array}{l}\text { Drought } \\
\text { sequence }\end{array}$} & \multirow{3}{*}{$\begin{array}{l}\text { First }^{*} \\
\text { day of } \\
\text { drought }\end{array}$} & \multirow{3}{*}{$\begin{array}{l}\text { Treat- } \\
\text { ment }\end{array}$} & \multirow{3}{*}{$\begin{array}{l}\text { Refe- } \\
\text { rence }\end{array}$} & \multirow{3}{*}{$\begin{array}{l}\text { Number } \\
\text { of data } \\
\text { days }\end{array}$} & \multicolumn{3}{|c|}{ Mean noon-time weather } \\
\hline & & & & & $\begin{array}{l}\text { Solar } \\
\text { radiation }\end{array}$ & $\begin{array}{l}\text { Air } \\
\text { temperature }\end{array}$ & $\begin{array}{l}\text { Dew-point } \\
\text { temperature }\end{array}$ \\
\hline & & & & & $\mathrm{W} / \mathrm{m}^{2}$ & ${ }^{\circ} \mathrm{C}$ & ${ }^{\circ} \mathrm{C}$ \\
\hline $1 / 1998$ & 30 July & TND & TNI & 3 & 906 & 31.0 & 22.2 \\
\hline 2/1998 & 3 Aug & TND & TNI & 6 & 804 & 31.4 & 25.0 \\
\hline 3/1998 & 12 Aug & TND & TNI & 5 & 861 & 31.0 & 23.0 \\
\hline 4/1998 & 14 Sept & TND & TNI & 5 & 696 & 29.5 & 22.5 \\
\hline $1 / 1999$ & 18 May & TND & E & 5 & 868 & 26.1 & 16.5 \\
\hline 3/1999 & 30 May & TND & $\mathrm{E}$ & 6 & 933 & 25.2 & 18.5 \\
\hline 4/1999 & 7 June & TND & $\mathrm{E}$ & 4 & 899 & 27.0 & 20.0 \\
\hline 3/1999 & 30 May & TPD & $\mathrm{E}$ & 6 & 933 & 25.2 & 18.5 \\
\hline 4/1999 & 7 June & TPD & E & 4 & 899 & 27.0 & 20.0 \\
\hline
\end{tabular}

* Day 2 in Figures T5.1 to T5.3.

\section{Results}

Canopy temperature and evaporation rate are shown as functions of time for each drought sequence in Figures T5.1 and T5.2, respectively. The temperature differences of Figure T5.1 were calculated as $\left(\mathrm{T}_{\mathrm{cs}}-\mathrm{T}_{\mathrm{as}}\right)-\left(\mathrm{T}_{\mathrm{cr}}-\mathrm{T}_{\mathrm{ar}}\right)$, namely as the difference between canopy-to-air temperature differences. Since in 1998 the stressed (subscript s) and reference (r) plants were in different chambers, the data for that year are not exactly what the symbols on the ordinate indicate, because $\left(\mathrm{T}_{\mathrm{cs}}-\mathrm{T}_{\mathrm{as}}\right)-\left(\mathrm{T}_{\mathrm{cr}}-\mathrm{T}_{\mathrm{ar}}\right) \neq \mathrm{T}_{\mathrm{cs}}-\mathrm{T}_{\mathrm{cr}}$. Note also that the reference in 1998 was irrigated tomato plants and in 1999 - an evaporimeter. 


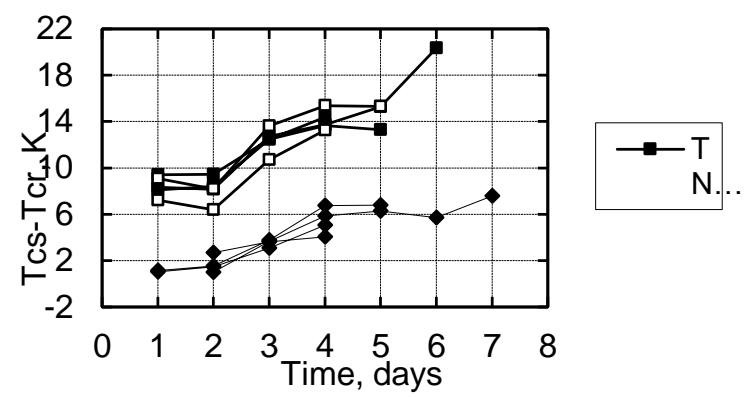

Figure T5.1: Noon-time (10:00 to 14:00 IST) tomato canopy-temperature elevation, $\mathrm{T}_{\mathrm{cs}}-\mathrm{T}_{\mathrm{cr}}$ (treatment minus reference), for the 1998 and 1999 tests. The reference in 1998 was irrigated tomato plants and in 1999 - an evaporimeter. TND and TPD are as in Table T5.1.

Figure T5.1 shows the following:

1 The temperature of the stressed plants increased with the buildup of stress. Irrigation was interrupted between days 1 and 2 and the signs of stress showed very clearly on day 3 (a jump of about $4 \mathrm{~K}$ in 1999), increasing further more slowly, up to about 5-6K. The temperature elevation, $\mathrm{T}_{\mathrm{cs}}-\mathrm{T}_{\mathrm{cr}}$, is a function of weather, as is demonstrated by the last points of the long sequences (2/1998 and 1/1999), which happened to be very hot and dry days.

2 The difference between the normal and pruned plants (in 1999) is less than the scatter within replications (mainly due to weather and plant variation). This means that removing the lower half-canopy had no significant effect on the temperature of the sunlit leaves.

3 The much higher differences for 1999 are due to the very effective evaporative cooling of the evaporimeter (Chapter T2), which was the reference for that year.

Figure T5.2, which is almost a mirror image of Figure T5.1, shows the following:

1 The first day with no irrigation (Day 2) shows no decline of transpiration, but the decline on day 3 is dramatic, to less than half of the original rate. Note that the availability of transpiration data does not exactly overlap the availability of temperature data (due to technical difficulties).

2 Again, the pruned plants could not be distinguished from the normal plants.

3 The absolute value of $\mathrm{R}$ is not meaningful when an evaporimeter is used as reference. The level of $\mathrm{R}$ on the first two days in the 1998 experiment was expected to be equal to 1 , an expectation fulfilled within the experimental margins of error. 


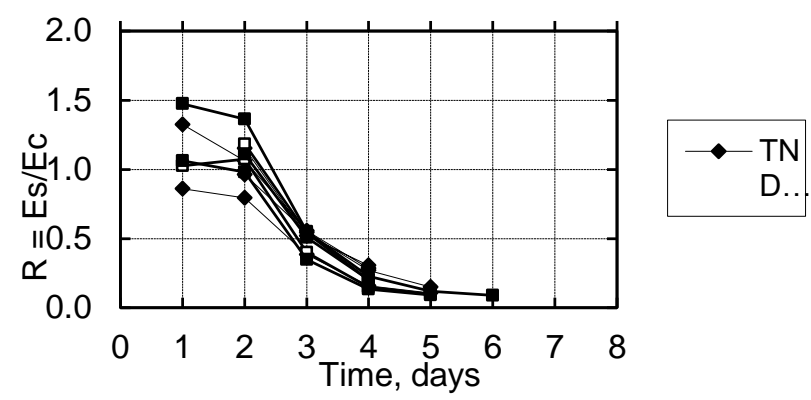

Figure T5.2: Noon-time (10:00 to $14: 00$ IST) evaporation ratio, $\mathrm{R} \equiv \mathrm{E}_{\mathrm{s}} / \mathrm{E}_{\mathrm{r}}$ (treatment divided by reference), for the 1998 and 1999 tests (linear R scale). The reference in 1998 was irrigated tomato plants and in 1999 - an evaporimeter. TND and TPD are as in Table T5.1.

Figure T5.3 only differs from Figure T5.2 in the logarithmic ordinate scale. It shows that the reduction of transpiration is roughly by a constant fraction (about 60\%) each day.

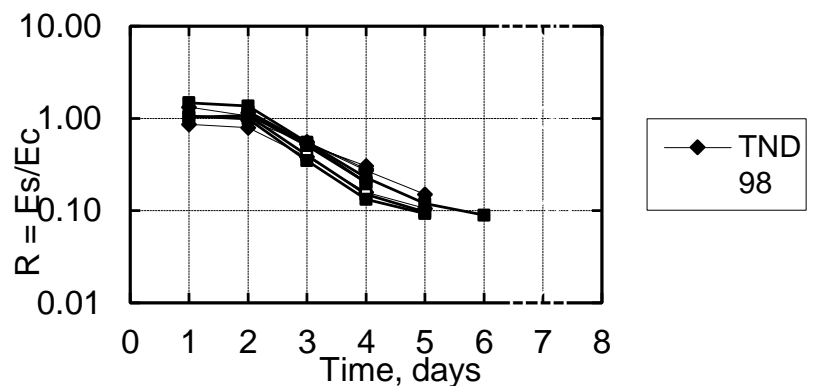

Figure T5.3: Noon-time (10:00 to 14:00 IST) evaporation ratio, $\mathrm{R} \equiv \mathrm{Es} / \mathrm{Er}$ (treatment divided by reference), for the 1998 and 1999 tests (logarithmic R scale). The reference in 1998 was irrigated tomato plants and in 1999 - an evaporimeter. TND and TPD are as in Table T5.1.

The mirror images of Figures T5.1 and T5.2 suggest the plotting of $\mathrm{T}_{\mathrm{cs}}-\mathrm{T}_{\mathrm{cr}}$ directly against $\mathrm{E}_{\mathrm{s}} / \mathrm{E}_{\mathrm{r}}$, as is shown in Figure T5.4. The initial points of each sequence are at the lower-right end of the curves. Generally speaking, the relationships are linear, with some tendency for concavity (upwards). Again, there is no difference between the normal and pruned tomato plants. 


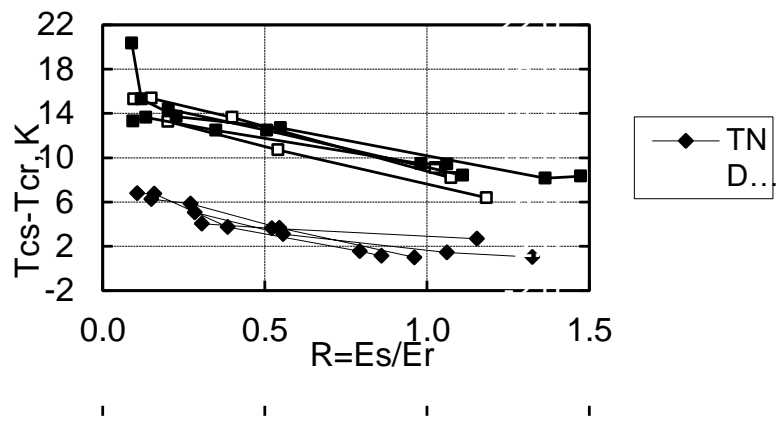

Figure T5.4: Relationship between the transpiration ratio, $\mathrm{R}=\mathrm{E}_{\mathrm{s}} / \mathrm{E}_{\mathrm{r}}$, and canopy temperature difference, $\mathrm{T}_{\mathrm{cs}}-\mathrm{T}_{\mathrm{cr}}$.

\section{Discussion}

The results of the preceding section depend on the particular weather-crop-pot combination of this study. Evidently, much of the soil moisture was extracted on the first day of drought (Day 2), so that the plants on the second day of drought (Day 3) were definitely stressed. We have some evidence, which should not be surprising, that the onset of stress takes longer to appear for less vigorously transpiring plants. Perhaps a more proper standard test for the time to onset of stress should involve a container that is large enough to approximate an infinite volume of substrate. In such a case, the limiting factor for water uptake would be the rate of growth of roots towards the remaining water. The current test mimics a situation where the plants cannot be considered solitary, because the water available for each plant is limited (implicitly by competing neighbors).

On the question of which is the best indicator of stress, the answer, based on the almost linear relationships of Figure T5.4, is that there is no clear advantage in principle to either the temperature- or the transpiration-based method. The more convenient of the two (depending on the situation) should be selected. Regarding the proper choice of reference, despite the different sensitivity of plants and evaporimeter to radiation and vapor pressure deficit (the two environmental attributes in the Penman-Monteith equation), the results of 1999 seem to show that the evaporimeter is a useful reference instrument. It cannot be assumed, however, that the plant-to-reference relationship is invariant with the weather. A practical method has to involve an adaptive model, which can predict $\mathrm{T}_{\mathrm{cs}}-\mathrm{T}_{\mathrm{cr}}\left(\right.$ or $\left.\mathrm{E}_{\mathrm{s}} / \mathrm{E}_{\mathrm{r}}\right)$ of an unstressed plant for the current weather and operating conditions. The measured value will have to be compared with the prediction before a stress warning can be issued.

The pruned plants were tested to see if plants with a large root-to-shoot ratio could last longer under drought conditions. The result, in terms of temperature (of the top leaves) and of total transpiration, was that apparently the lower half of the canopy of the intact plants did not transpire, at least not significant amounts of water. If more leaves are removed, obviously a point is reached where the transpiration rate of the plant as a whole would decrease. Perhaps a more useful measure of transpiration could be based on the sunlit leaf area (=shaded ground area), rather than on total remaining leaf area. Results at Newe Ya'ar (Chapters Y1 and Y2) showed some advantage to pruned plants. 


\title{
Y1 THE EFFECT OF ROOT/SHOOT RATIO ON SPECIFIC TRANSPIRATION OF GROWTH CHAMBER GROWN TOMATO TRANSPLANTS
}

\author{
Michael Raviv, Shlomit Medina, Arkady Krasnovsky \\ Department of Ornamental Horticulture, ARO, Newe Ya'ar
}

\section{Abstract}

Tomato seedlings having different root/shoot $(\mathrm{R} / \mathrm{S})$ ratio were produced using leaf removal prior to the beginning of the experiments. Otherwise the plants were treated identically. Half of the plants were exposed to gradually increasing water stress, during 3 consecutive days while the other half were maintained at container capacity. The experiments were conducted in a growth chamber, under high temperature $\left(35^{\circ} \mathrm{C}\right)$. The experiments were replicated trice. Irrigated plants grew faster (as expressed in their daily relative growth rate - RGR) than nonirrigated plants. Pruned plants had higher RGR than normal plants. When calculated per plant, transpiration rates did not differ greatly among the treatments until the third day of water withholding, when moisture content (MC) dropped to 45\%. However, when calculated per leaf area, high R/S plants had higher specific transpiration rate (STR) than low R/S plants. This enabled non-irrigated plants having high $\mathrm{R} / \mathrm{S}$ ratio to maintain lower leaf temperatures than their low R/S counterparts. When calculated on a root area basis (third day only) both irrigated and non-irrigated plants had similar STR. This suggests that at all moisture regimes the canopy can benefit from a more favorable R/S ratio, probably due to the fact that the root surface is one of the limiting factors for water flux through the system.

\section{Introduction}

One of the goals, set for the third year of this research, was to try to devise a standard method for assessing transplants capability to cope with stressful conditions. In doing so, we had to accommodate for two contrasting requirements: On the one hand, it is important to conduct such test under well-defined and reproducible conditions. This requirement dictates the use of a growth chamber where constant, pre-determined conditions can be maintained. On the other hand, light intensity, which is the main cause of high leaf temperature, is relatively low in a growth chamber, comparing to greenhouse conditions. We tried to overcome this difficulty by deliberately providing conditions of high temperature (and consequently, high VPD). The results presented in the previous report suggest that, indeed, stress can be induced under such conditions and that plants having high root/shoot ratio were better equipped to cope with the imposed stress.

\section{Materials and Methods}

In our first two annual reports to BARD, two procedures for producing sweet pepper and tomato seedlings, having various ratios of root/shoot weights were described. These procedures were based on withholding water or mineral nutrition. These procedures yielded plants that are not typical to regular nursery-grown transplants. In the current year we chose to apply another method to alter the root/shoot ratio: all the plantlets were grown under normal, identical conditions. A short while before starting the measurements, 2-3 of the lowermost leaves (30-50\% of the total leaf area) were removed. This was done 3-24 hours before starting the experiments. 
Tomato (CV. F-144, "Daniella") seedlings were sown in peat:vermiculite (\#3) (1:1)-filled "speedling" trays. The seeds were germinated for 7 days in a growth chamber $\left(25^{\circ} \mathrm{C}\right.$, $60 \% \pm 5 \% \mathrm{RH}, 80 \mu \mathrm{mol} \mathrm{m} \mathrm{m}^{-2} \mathrm{~s}^{-1}$ ), until the cotyledons were unfolded. At this time they were transferred to a greenhouse for additional week. The trays were sub-irrigated with a 20:20:20 $\left(\mathrm{N}: \mathrm{P}_{2} \mathrm{O}_{5}: \mathrm{K}_{2} \mathrm{O}\right)$ solution, based on $200 \mathrm{ppm} \mathrm{N}$. At this stage, the plantlets were transferred, 3 per pot, to 0.5 -liter pots, filled with the same medium. The plants were grown in these pots for 2 weeks. Nutrition during this time was based on $150 \mathrm{ppm} \mathrm{N}$ and K, $30 \mathrm{ppm} \mathrm{P}$ and a complete chelated microelement solution. When the plants reached a height of about $30 \mathrm{~cm}$, the plants were transferred to a growth chamber and the experiments were initiated a day later. Conditions during the experiment were $35^{\circ} \mathrm{C}, 80 \% \pm 5 \% \mathrm{RH}, 180 \mu \mathrm{mol} \mathrm{m} \mathrm{m}^{-2} \mathrm{~s}^{-1}$. In each case, plants were either left with their initial leaf area or 2 - 3 lowermost leaves were removed. Leaf area of all plants was measured. The length and width of each leaf were measured and their product was used to calculate leaf area according to a previously prepared calibration curve. Within each group, half of the plants were irrigated daily, to saturation. When drainage stopped, the pots were left on a saucer with water. Water level was above the drainage holes, to ensure that the plants will stay at container capacity during the whole measurement period. Weighing was started when all pots reached their container capacity. The other half was left without watering for 3 days. The media were wrapped with aluminum foil to minimize heating and evaporation. Leaf temperatures were recorded using thermocouples (2 per treatment) glued to the lower side of the upper leaves. The results are presented as averages of the temperatures of the 2 leaves, over the daytime hours.

At the end of the experiment leaf area was determined again. Root surface area was measured according to the following procedure: Forty $1-\mathrm{cm}$ functional root segments were sampled. For each segment the accurate length and diameter were measured, so that surface area could be calculated. The segments were weighed and a ratio of $85 \mathrm{~cm}^{2} \mathrm{~g}^{-1}$ was determined. Subsequently, all roots were removed and functional and nonfunctional parts were separated. It was found that the ratio of functional to total weight is 0.84 . Using these data, root surface area was later calculated from fresh weight data.

\section{Results and Discussion}

The experiment was replicated 3 times with 3 pots per treatment and 3 plants per pot at each run. Plant data of a representative experiment are presented in Table Y1.1.

Table Y1.1: Initial leaf area, daily relative growth rate (RGR) based on leaf area, final leaf and root area and R/S ratio at the end of the experiment. (Average of 9 plants per treatment).

\begin{tabular}{|l|c|c|c|c|c|}
\hline Treatment & $\begin{array}{c}\text { Initial leaf } \\
\text { area }\left(\mathrm{cm}^{2}\right)\end{array}$ & $\begin{array}{c}\text { Final leaf area } \\
\left(\mathrm{cm}^{2}\right)\end{array}$ & $\begin{array}{c}\text { Daily RGR } \\
(\%)\end{array}$ & $\begin{array}{c}\text { Root area } \\
\left(\mathrm{cm}^{2}\right)\end{array}$ & $\begin{array}{c}\mathrm{R} / \mathrm{S} \\
\text { ratio }\end{array}$ \\
\hline Low R/S, irr. & 2913 & 3504 & 9.7 & 2824 & 0.88 \\
\hline $\begin{array}{l}\text { Low R/S, non- } \\
\text { irr. }\end{array}$ & 2599 & 2552 & -0.6 & 2589 & 1.01 \\
\hline High R/S, irr. & 1846 & 2404 & 14.1 & 2464 & 1.16 \\
\hline $\begin{array}{l}\text { High R/S, non- } \\
\text { irr. }\end{array}$ & 1889 & 1946 & 1.5 & 2356 & 1.23 \\
\hline
\end{tabular}

Non-irrigated plants hardly increased their leaf area while leaf area of irrigated plants developed at a daily RGR of 9.7-14.1\%. It is noteworthy that in both cases, the plants having initial lower leaf area showed higher RGR values. If such a trend persists long enough, this 
may suggest that under normal greenhouse conditions, without water stress, the high R/S plants will eventually compensate for the leaf removal.

Assuming that prior to the leaf removal, the R/S ratios of all treatments were similar, it seems that non-irrigated plants accelerated their root growth, as reflected in their higher R/S values as compared to their irrigated counterparts. The change in containers' weight over the duration of the experiment is shown in Figure Y1.1.

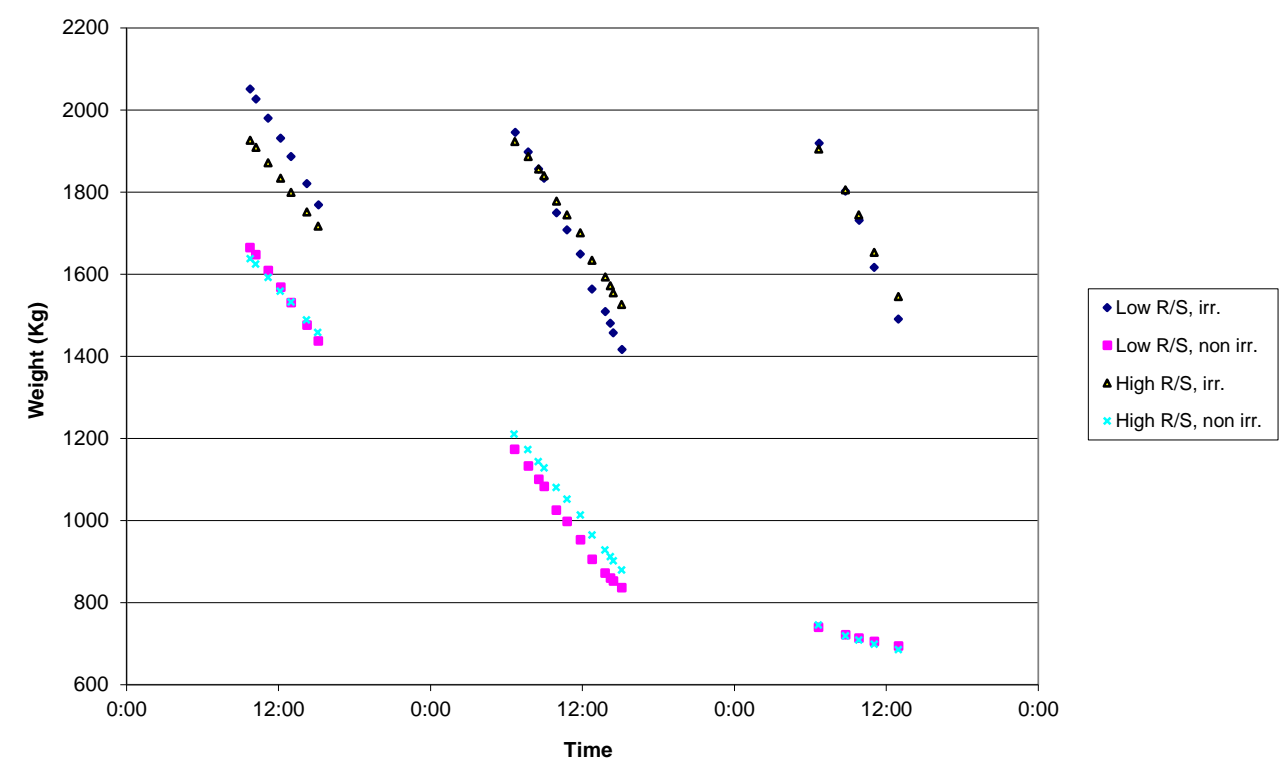

Figure Y1.1: Average weight loss of plants of the 4 treatments during a 3-day experiment.

The slopes (namely, rate of water loss) vary slightly (but significantly, see low R/S irrigated vs. non-irrigated plants in Table Y1.2) among all cases (treatments and days) except for the last day of the non-irrigated plants. It can also be seen that the root/shoot ratio hardly affected the rate of loss of container weight. Plants having reduced leaf area apparently transpired at the same rate as their undisturbed counterparts. This may result from either of two factors or from their combination: Their fewer leaves transpired at a higher rate per unit leaf area, as can be seen in Table Y1.2. Or, that their older, more shaded leaves contribute an unproportional low contribution to the total transpiration, in respect to their leaf area.

Table Y1.2: Average specific transpiration rate (STR, $\left.\mathrm{g} \mathrm{m}^{-2} \mathrm{~h}^{-1}\right)$ of plants of the 4 treatments along the 3-day experiment. Different letters in the same column denote statistically significant values at $\mathrm{P}<0.05$, according to Tukey-Kramer HSD.

\begin{tabular}{|l|c|c|c|}
\hline Treatment & Day 1 & Day 2 & Day 3 \\
\hline Low R/S, irr. & $180 \mathrm{~b}$ & $203 \mathrm{a}$ & $203 \mathrm{a}$ \\
\hline Low R/S, non-irr. & $162 \mathrm{c}$ & $147 \mathrm{~b}$ & $28 \mathrm{~b}$ \\
\hline High R/S, irr. & $211 \mathrm{a}$ & $232 \mathrm{a}$ & $246 \mathrm{a}$ \\
\hline High R/S, non-irr. & $179 \mathrm{~b}$ & $203 \mathrm{a}$ & $48 \mathrm{~b}$ \\
\hline
\end{tabular}

As expected, large variation exists among the STR values of the 4 treatments. The variation was less clear at the first day, when the non-irrigated containers had still high moisture content. The normal irrigated plants transpired more than non-irrigated plants, per leaf area. 
Non-irrigated, high R/S plants transpired as much as irrigated low R/S plants for 2 days. Only in the third day of water withholding, their STR value dropped dramatically. This suggests that changing the $\mathrm{R} / \mathrm{S}$ can delay severe damage to plants exposed to water shortage (and presumably also to increased atmospheric demand). The effect of moisture content (MC) in the medium is shown in Figure Y1.2.

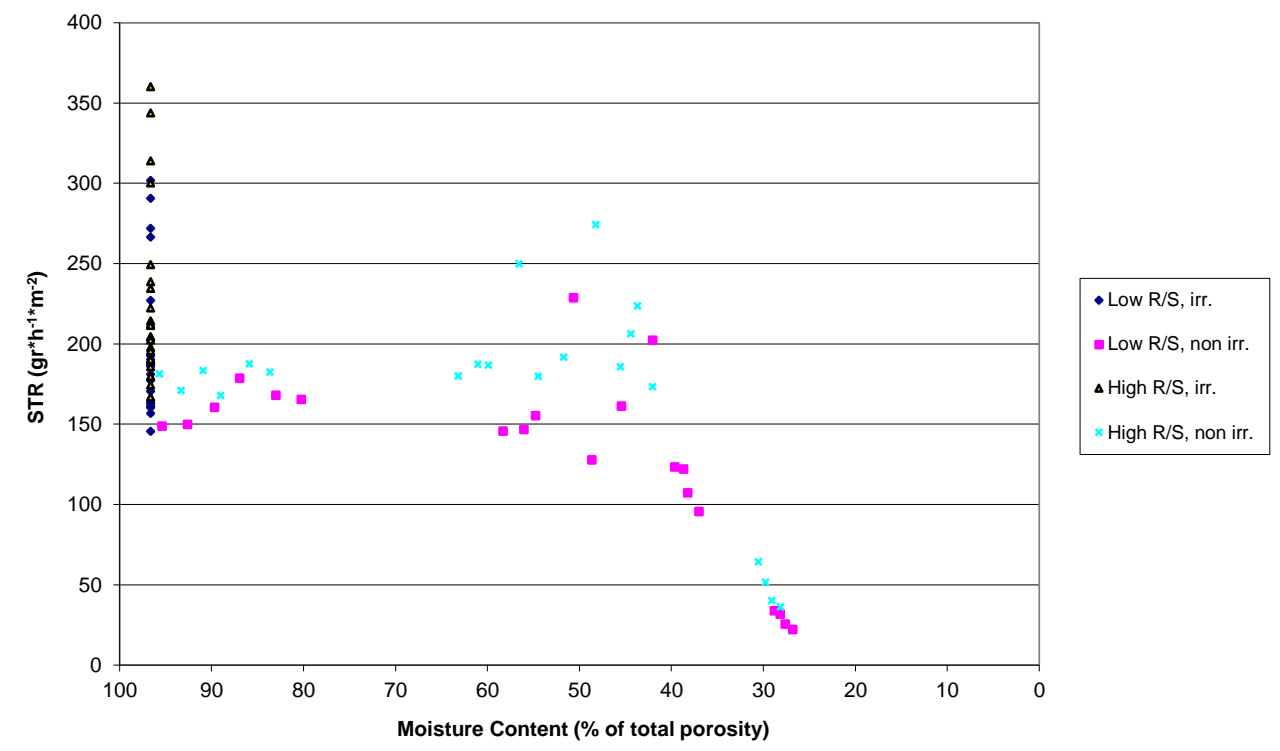

Figure Y1.2: Average STR of plants of the 4 treatments, as a function of moisture content of the medium.

When plotted against MC, STRs of plants of the two non-irrigated treatments were different from each other in most of the relevant range (95-45\% of total porosity). Within this range, and at the same MC, high R/S plants had consistently higher STR than low R/S plants. At MC below $45 \%$, no difference could be noticed. Specific root water uptake is shown in Table Y1.3.

Table Y1.3: Specific root uptake rate $\left(\mathrm{g} \mathrm{m}^{-2} \mathrm{~h}^{-1}\right)$ during the third day. Different letters in the same column denote statistically significant values at $\mathrm{P}<0.05$, according to Tukey-Kramer HSD.

\begin{tabular}{|l|c|}
\hline Treatment & Specific root uptake \\
\hline Low R/S, irr. & $252 \mathrm{a}$ \\
\hline Low R/S, non-irr. & $28 \mathrm{~b}$ \\
\hline High R/S, irr. & $240 \mathrm{a}$ \\
\hline High R/S, non-irr. & $40 \mathrm{~b}$ \\
\hline
\end{tabular}

For obvious reasons, we have reliable data as to the active root surface area only for the last day of the experiment. At container capacity there was no difference between specific root uptake rates of the plants having high or low root/shoot ratio. This suggests that even at this ideal moisture regime the canopy can benefit from a more favorable R/S ratio, probably due to the fact that the root surface is a limiting factor for water flux through the system. On the third day, the moisture content of the non-irrigated plants dropped to about $30-25 \%$ of the 
total porosity. At this low content of available water the specific root uptake dropped 9-6 fold for the normal and pruned plants, respectively.

The effect of the treatment on leaf temperature can be seen in Table Y1.4.

Table Y1.4: Average leaf temperatures of the 4 treatments during the 3 days of the experiment. Different letters in the same column denote statistically significant values at $\mathrm{P}<0.05$, according to Tukey-Kramer HSD.

\begin{tabular}{|l|l|l|l|}
\hline Treatment & Day 1 & Day 2 & Day 3 \\
\hline Low R/S, irr. & $34.0 \mathrm{a}$ & $32.6 \mathrm{a}$ & $34.0 \mathrm{a}$ \\
\hline Low R/S, non-irr. & $35.3 \mathrm{~b}$ & $36.9 \mathrm{c}$ & $38.2 \mathrm{c}$ \\
\hline High R/S, irr. & $34.0 \mathrm{a}$ & $32.6 \mathrm{a}$ & $33.7 \mathrm{a}$ \\
\hline High R/S, non-irr. & $35.0 \mathrm{~b}$ & $34.4 \mathrm{~b}$ & $35.9 \mathrm{~b}$ \\
\hline
\end{tabular}

Air velocity within the growth chambers is very high. Therefore the temperatures of the irrigated plants' leaves were only $1-2^{\circ} \mathrm{C}$ below the air temperature. This consistent temperature gradient can serve as an indication for a normal transpiration apparatus. No temperature difference can be found between irrigated plants having high and low R/S. On the other hand, a clear difference between the leaf temperatures of the plants having high and low $\mathrm{R} / \mathrm{S}$ was found among the non-irrigated plants, in the second and third day. This, again, demonstrates the effectiveness of leaf removal as a way to moderate water stress damage to seedlings.

\section{Conclusions}

The outlined goal, as presented in the proposal was achieved. It seems that a rather quick and uniform test for transplants ability to cope with environmental stress can be performed under controlled conditions. Any of the measured parameters (STR, rate of weight loss, leaf temperature) can be used as a real-time indicator for the onset of stress. Such a test may have practical value if its results correspond with the plants' performance under greenhouse conditions. This is the subject of the next chapter.

The increased RGR of plants with reduced leaf area (Table Y1.1) probably resulted from the improved conditions of the remaining canopy. This may suggest that once the initial postplanting stress is over, plants can compensate for moderate leaf removal.

The similar transpiration rate of plants having high and low R/S ratio (Figure Y1.1) suggests that aboveground factors such as boundary layer resistance are not the limiting factors for specific transpiration rate. Similarity in transpiration rates between pruned and normal plants may result from either of two factors or from their combination: The fewer leaves of pruned plants transpired at a higher rate per unit leaf area, as can be seen in Table Y1.2. Or, the older, more shaded leaves of normal plants contribute an unproportional low contribution to the total transpiration, in respect to their leaf area. 


\title{
Y2 THE EFFECT OF ROOT/SHOOT RATIO ON SPECIFIC TRANSPIRATION OF GREENHOUSE-GROWN TOMATO TRANSPLANTS
}

\author{
Michael Raviv, Shlomit Medina, Arkady Krasnovsky \\ Department of Ornamental Horticulture, ARO, Newe Ya'ar
}

\section{Abstract}

Tomato seedlings having different root/shoot $(\mathrm{R} / \mathrm{S})$ ratio were produced using leaf removal prior to the beginning of the experiments. Otherwise the plants were treated identically. Half of the plants were exposed to gradually increasing water stress, during 3 consecutive days while the other half were maintained at container capacity. The experiments were conducted in a greenhouse, under summer conditions, without cooling. The experiments were replicated trice. Irrigated plants grew faster (as expressed in their daily relative growth rate - RGR) than non-irrigated plants. Plants having high R/S ratio had higher RGR than plants having low RGR. When calculated per plant, transpiration rates of the two irrigated treatments were quite similar. This suggests that under conditions of ample water supply canopy of high R/S plants is better equipped to cope with high atmospheric demand for water. Among non-irrigated plants, the differences were minor until the third day of water withholding. At this point high $\mathrm{R} / \mathrm{S}$ plants transpired more than low R/S plants. When calculated on a leaf area basis, nonirrigated high R/S plants had consistently higher specific transpiration rate (STR) than low $\mathrm{R} / \mathrm{S}$ plants. Non-irrigated plants having high R/S ratio maintained lower leaf temperatures than their low R/S counterparts only in the third day. When calculated on a root area basis (third day only) the two irrigated treatments had similar STR. This may suggest that at all moisture regimes the canopy can benefit from a more favorable R/S ratio, probably due to the fact that the root surface is the limiting factor for water flux through the system. Another possible explanation to these results is that lower, shaded leaves contribute an unproportional low contribution to the total transpiration, in respect to their leaf area. Non-irrigated, high R/S plants had higher root STR than low R/S, probably due to the higher moisture content (MC) of this treatment.

\section{Introduction}

The applicability and reliability of the bioassay, conducted in growth chamber was tested in 3 summer greenhouse experiments. The greenhouse aerial conditions were not moderated using evaporative cooling in order to simulate the conditions that prevail in a sparsely planted greenhouse, soon after planting. The results obtained in these replicated experiments were quite uniform and a representative example is shown here.

\section{Materials and Methods}

The plant material for the experiments was prepared as described in Chapter Y1, except for the last stage - when the plants were ready; the experiments were initiated in the same greenhouse and were not transferred to a growth chamber. The conditions within the greenhouse during the 3 days of experiment were $18-36^{\circ} \mathrm{C}$ and $85-45 \% \mathrm{RH}$ (night/day). The measured parameters and treatment to the plant material were identical to the previous experiment. Results are presented for the time interval 11:00-15:00, when VPD was high and relatively uniform. 
Net photosynthesis was measured using a portable photosynthesis meter (Li-Cor 6200, Lincoln, NE). Only non-irrigated plants were sampled, during the second and third day of the experiments.

\section{Results}

The measured plants parameters are shown in Table Y2.1.

Table Y2.1: Initial leaf area, daily relative growth rate (RGR) based on leaf area, final leaf and root area and R/S ratio at the end of the experiment. (Average of 9 plants per treatment).

\begin{tabular}{|l|c|c|c|c|c|}
\hline Treatment & $\begin{array}{c}\text { Initial leaf } \\
\text { area }\left(\mathrm{cm}^{2}\right)\end{array}$ & $\begin{array}{c}\text { Final leaf area } \\
\left(\mathrm{cm}^{2}\right)\end{array}$ & $\begin{array}{c}\text { Daily RGR } \\
(\%)\end{array}$ & $\begin{array}{c}\text { Root area } \\
\left(\mathrm{cm}^{2}\right)\end{array}$ & $\begin{array}{c}\mathrm{R} / \mathrm{S} \\
\text { ratio }\end{array}$ \\
\hline Low R/S, irr. & 886 & 1453 & 28.0 & 1411 & 0.97 \\
\hline $\begin{array}{l}\text { Low R/S, non- } \\
\text { irr. }\end{array}$ & 818 & 1020 & 11.7 & 1136 & 1.11 \\
\hline High R/S, irr. & 513 & 928 & 34.6 & 1035 & 1.12 \\
\hline $\begin{array}{l}\text { High R/S, non- } \\
\text { irr. }\end{array}$ & 408 & 699 & 30.9 & 1214 & 1.74 \\
\hline
\end{tabular}

RGR values of greenhouse-grown plants were much higher than those of the growth chambergrown plants. The observed trends, however, were similar, namely higher RGR of irrigated and high R/S ratio plants $v s$. non-irrigated and low R/S plants, respectively. Non-irrigated plants accelerated their root growth, as reflected in their higher R/S values as compared to their irrigated counterparts even more than in the growth chamber. Average weight loss of the four treatments is presented in Figure Y2.1.

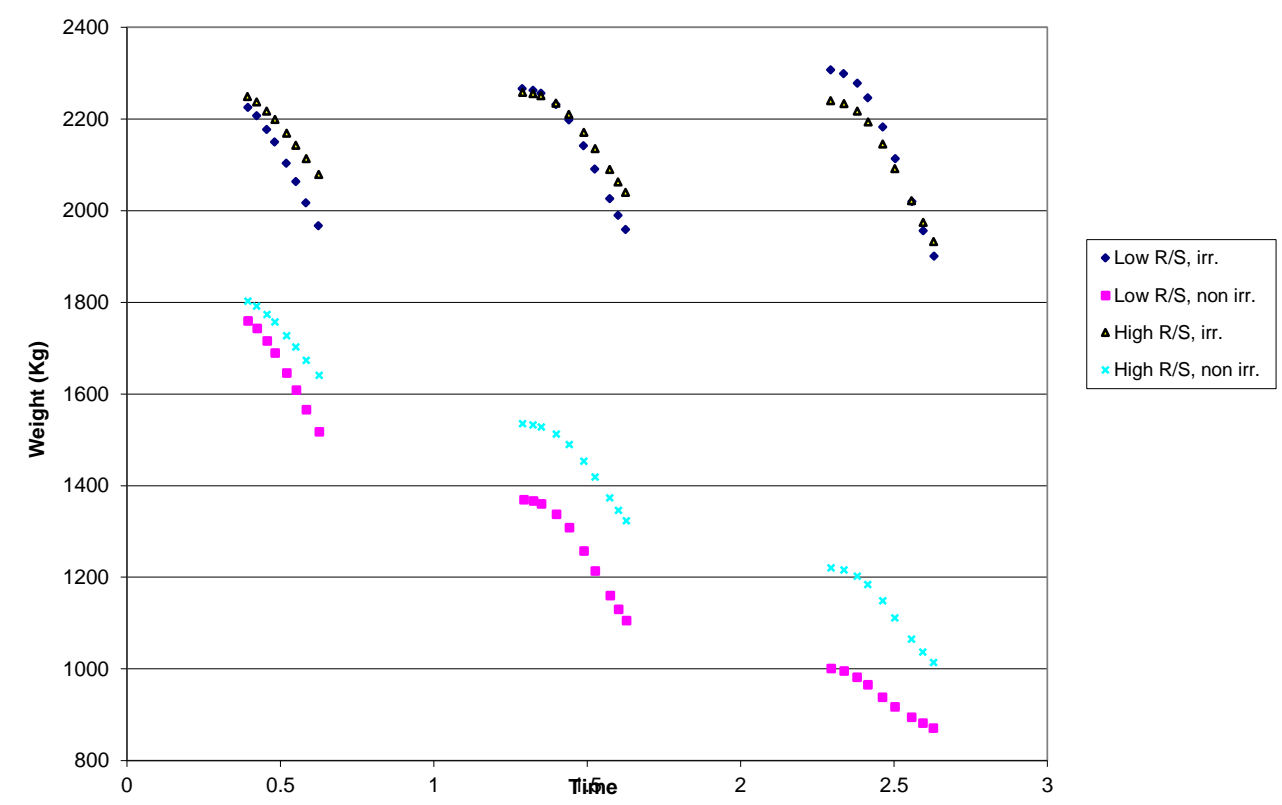

Figure Y2.1: Average weight loss of plants of the 4 treatments during 3 days of the experiment. Daily measurement period: 11:00-15:00. 
The slopes (namely, rate of water loss) of the various treatments were less homogenous than those of the growth chamber-grown plants, both during the day and among treatments. Daily variations result from the fact that PPFD, temperature and $\mathrm{RH}$ in the greenhouse are much less uniform than in the growth chamber. Variations among treatments probably result from the much higher atmospheric demand for water, characterizing the greenhouse daily conditions, as compared to the conditions within the growth chamber. The most interesting trend is that of low vs. high R/S, non-irrigated plants. In the first day, when MC is high, low $\mathrm{R} / \mathrm{S}$ transpired faster than high R/S plants, as can be expected due to their higher leaf area. In the second day their slopes were similar while during the third day, high R/S plants transpired more than low R/S plants, which is counterintuitive. This apparently strange phenomenon resulted from their higher MC. In the second and third day, the root/shoot ratio of irrigated plants hardly affected the rate of loss of container weight. Plants having reduced leaf area apparently transpired at the same rate as their undisturbed counterparts. This necessarily means that their fewer leaves transpired at a higher rate per unit leaf area. This fact is clearly shown in Table Y2.2.

Table Y2.2: Average specific transpiration rate (STR, $\left.\mathrm{g} \mathrm{m}^{-2} \mathrm{~h}^{-1}\right)$ of plants of the 4 treatments along the 3-day experiment (11:00-15:00). Different letters in the same column denote statistically significant values at $\mathrm{P}<0.05$, according to TukeyKramer HSD.

\begin{tabular}{|l|c|c|c|}
\hline Treatment & Day 1 & Day 2 & Day 3 \\
\hline Low R/S, irr. & $558 \mathrm{a}$ & $477 \mathrm{a}$ & $461 \mathrm{~b}$ \\
\hline Low R/S, non-irr. & $563 \mathrm{a}$ & $491 \mathrm{a}$ & $177 \mathrm{a}$ \\
\hline High R/S, irr. & $630 \mathrm{ab}$ & $561 \mathrm{a}$ & $550 \mathrm{~b}$ \\
\hline High R/S, non-irr. & $756 \mathrm{~b}$ & $701 \mathrm{~b}$ & $470 \mathrm{~b}$ \\
\hline
\end{tabular}

Absolute STR values of all treatments were 3-4 times higher in the greenhouse than in the growth chamber (see chapter Y1, Table Y1.2). This is a combined result of higher energy load and VPD in the greenhouse. The initial low leaf area of the high R/S, non-irrigated plants, as well as their extremely high $\mathrm{R} / \mathrm{S}$ ratio (compared to their irrigated counterparts) resulted with very high STR values, especially during the first 2 days. Even at the third day, when the MC of these plants dropped to 40-50\% (Figure Y2.2), their STR was similar to this of both irrigated treatments. This suggests that changing the R/S of transplants just before planting can moderate and delay severe damage to them if exposed to water shortage (and presumably also to increased atmospheric demand). The effect of moisture content of the media is shown in Figure Y2.2.

When plotted against moisture content, STRs of plants of the two non-irrigated treatments were different from each other in most of the relevant range (95-40\% of total porosity). Within this range, and at the same moisture content, high R/S plants had consistently higher STR than low R/S plants. High R/S plants never reached MC values below $40 \%$. 


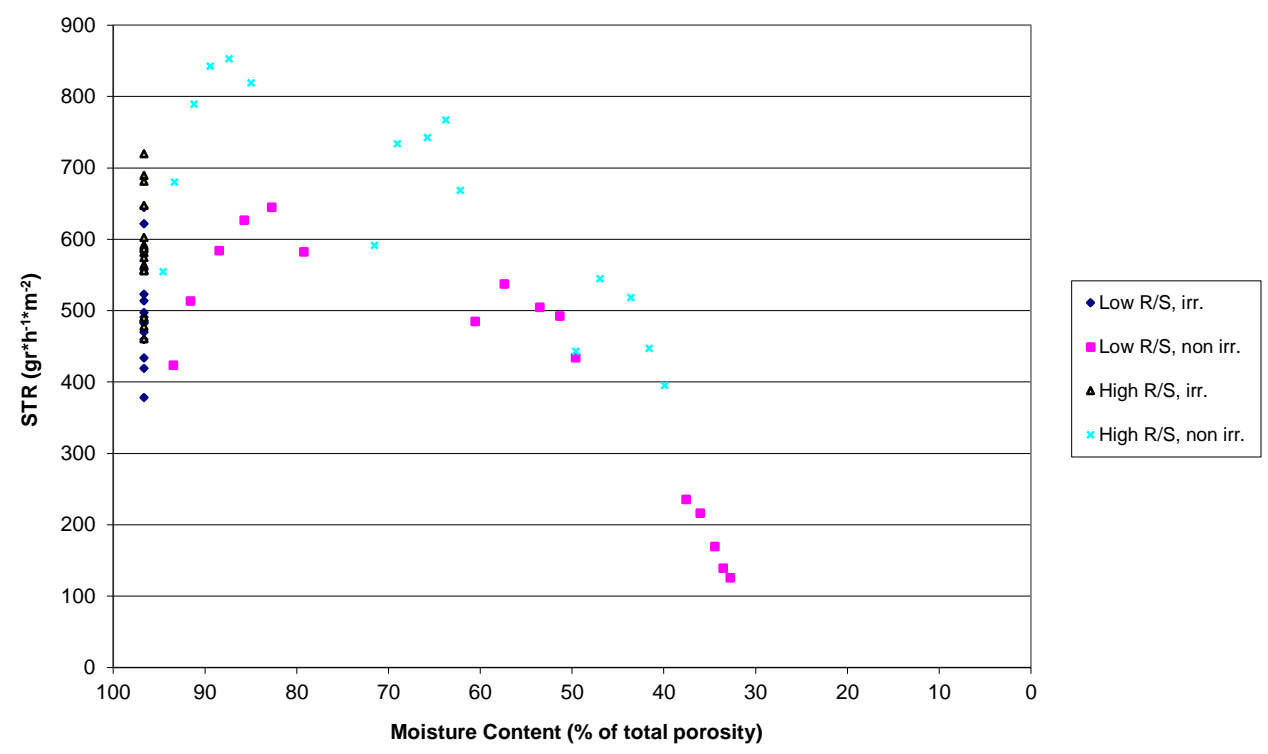

Figure Y2.2: Average STR of plants of the 4 treatments, as a function of MC of the media during the 3 days of the experiment (11:00-15:00).

Table Y2.3: Specific root uptake rate $\left(\mathrm{g} \mathrm{m}^{-2} \mathrm{~h}^{-1}\right)$ during the third day. Different letters in the same column denote statistically significant values at $\mathrm{P}<0.05$, according to Tukey-Kramer HSD.

\begin{tabular}{|l|c|}
\hline Treatment & Specific root uptake \\
\hline Low R/S, irr. & $370 \mathrm{c}$ \\
\hline Low R/S, non-irr. & $140 \mathrm{a}$ \\
\hline High R/S, irr. & $370 \mathrm{c}$ \\
\hline High R/S, non-irr. & $210 \mathrm{~b}$ \\
\hline
\end{tabular}

As for the growth chamber-grown plants, there was no difference between specific root uptake rates of the plants having high or low root/shoot ratio at container capacity. This suggests that even at this ideal moisture regime the canopy can benefit from a more favorable $\mathrm{R} / \mathrm{S}$ ratio, probably as a result of the root surface being one of the limiting factors for water flux through the system. On the third day the moisture content of the high R/S non-irrigated plants dropped to about $40-50 \%$ of the total porosity. The $\mathrm{MC}$ of the non-irrigated, low $\mathrm{R} / \mathrm{S}$ plants dropped to $30-40 \%$. This difference in MC was enough to induce a $50 \%$ difference in root STR between the two treatments. Similar trend was found for growth chamber-grown plants. Average leaf temperatures are presented in Table Y2.4.

Unlike in the case of growth chamber-grown plants, consistent temperature difference were found between irrigated plants having high and low R/S, suggesting that even under ample water supply young transplants may benefit (in terms of leaf temperature) from leaf removal. Surprisingly, the trend was less clear between the leaf temperatures of the non-irrigated plants and only in the third day $1.4^{\circ} \mathrm{C}$ difference was found. Still, this fact demonstrates the effectiveness of leaf removal as a way to moderate water stress damage to seedlings. The aerial conditions during these 3 days (11:00-15:00) were about $36^{\circ} \mathrm{C}, 35-45 \% \mathrm{RH}$. 
Table Y2.4: Average leaf temperatures of the 4 treatments during the 3 days of the experiment. Different letters in the same column denote statistically significant values at $\mathrm{P}<0.05$, according to Tukey-Kramer HSD.

\begin{tabular}{|l|c|c|c|}
\hline Treatment & Day 1 & Day 2 & Day 3 \\
\hline Low R/S, irr. & $33.1 \mathrm{a}$ & $34.0 \mathrm{a}$ & $35.9 \mathrm{~b}$ \\
\hline Low R/S, non-irr. & $32.3 \mathrm{~b}$ & $33.0 \mathrm{~b}$ & $37.3 \mathrm{a}$ \\
\hline High R/S, irr. & $32.0 \mathrm{~b}$ & $33.0 \mathrm{~b}$ & $33.7 \mathrm{c}$ \\
\hline High R/S, non-irr. & $33.1 \mathrm{a}$ & $33.0 \mathrm{~b}$ & $35.9 \mathrm{~b}$ \\
\hline
\end{tabular}

In another experiment leaf temperature data were slightly different. The data are presented in Table Y2.5.

Table Y2.5: Average leaf temperatures of tomato plants during 3 days of experiment. Different letters in the same column denote statistically significant values at $\mathrm{P}<0.05$, according to Tukey-Kramer HSD.

\begin{tabular}{|l|l|l|l|}
\hline Treatment & Day 1 & Day 2 & Day 3 \\
\hline High R/S, irr. & $32.4 \mathrm{a}$ & $32.8 \mathrm{a}$ & $35.0 \mathrm{~b}$ \\
\hline Low R/S, non-irr. & $32.4 \mathrm{a}$ & $33.0 \mathrm{a}$ & $37.6 \mathrm{a}$ \\
\hline High R/S, irr. & $32.6 \mathrm{a}$ & $33.2 \mathrm{a}$ & $33.6 \mathrm{c}$ \\
\hline High R/S, non-irr. & $33.1 \mathrm{a}$ & $33.3 \mathrm{a}$ & $35.8 \mathrm{~b}$ \\
\hline
\end{tabular}

In this case the aerial conditions during these 3 days (11:00-15:00) were much milder than in the previous run: about $33^{\circ} \mathrm{C}, 40-50 \% \mathrm{RH}$. Consequently, in the first two days, no difference was observed among the treatments, as was found in the growth chamber experiments. Only in the third day non-irrigated plants exhibited higher leaf temperatures than irrigated plants. Pruned, non-irrigated plants were $1.8^{\circ} \mathrm{C}$ cooler than the control plants. In the third day also the leaf temperature of pruned, irrigated plants was cooler than that of the control plants, as was found in the previous experiment. It is clear that the water atmospheric demand was much lower in this case and could be satisfied at a much lower MC (data not shown).

Although the data in Figure Y2.3 are very scattered, the calculated trend lines point to a clear superiority in terms of photosynthesis efficiency of the pruned plants. These results probably reflect the difference in leaf temperature and stomatal resistance (data not shown) between the normal and pruned plants. This $\sim 25 \%$ difference in net assimilation rate can provide some explanation to the difference in RGR between the normal and pruned plants.

\section{Conclusions}

The results of the greenhouse experiments suggest that the proposed growth chamber bioassay provides a reasonable prediction to the relative capability of tomato transplants to cope with water stress. 


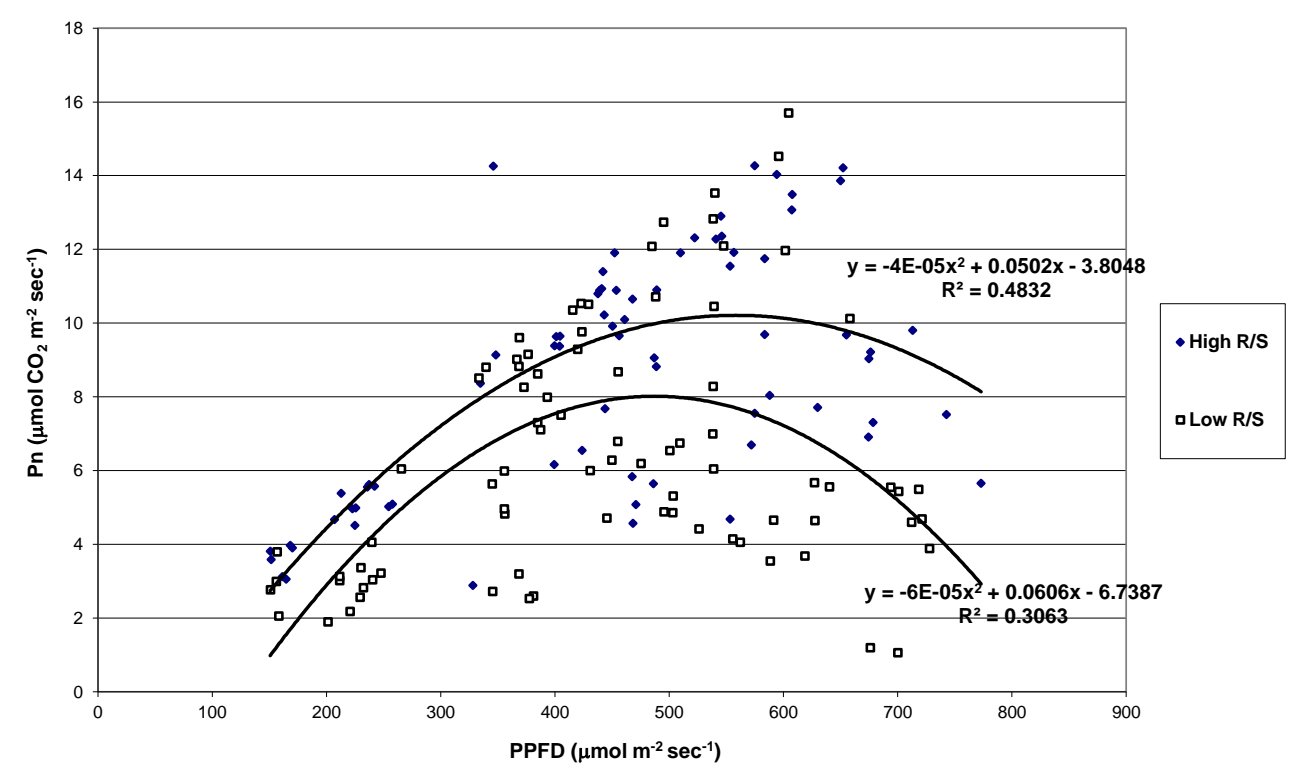

Figure Y2.3: Net photosynthesis of non-irrigated tomato plants as a function of PPFD. Cumulative of 3 experiments.

When compared to the control (low R/S) plants, irrigated high R/S plants exhibited higher RGR values, similar STR values (based on leaf and root area) and lower leaf temperatures. It can be concluded that under normal water supply and high atmospheric demand for water, such a pretreatment to transplants can be of practical value in moderating and delaying water stress damage. The predicted cost to growth rate should be minimal due to the higher RGR. If the current RGR values will persist, irrigated, pruned plants will reach the leaf area of normal plants in 9 days.

Under imposed drought conditions, RGR, STR and net assimilation rate values (based on whole plant, leaf and root basis) of high R/S plants were higher than those of the control treatment. Leaf temperature of the control plants was higher than that of the pruned plants, once the hydraulic conductivity of the medium/rhizoplane could not supply the water flux required by the atmospheric demand. 